\title{
4 INKLUSION UND ZUSAMMENLEBEN IN ÖSTERREICH
}

\subsection{Wohnen und Wohnungsmarkt}

\subsubsection{Empirischer Forschungsstand}

Das Wohnen repräsentiert einen wichtigen Aspekt der strukturellen Integration (Bosswick et al. 2007; EsSER 2001) und hat vielfältige Auswirkungen auf andere Integrationsbereiche wie Bildungschancen und Arbeitsmarktpositionierung (UNHCR 2013). „Integration beginnt im sozialen Umfeld eines Menschen, direkt vor Ort, in den Siedlungen in der Nachbarschaft, in den Gemeinden und Städten. Daher ist es besonders wichtig, dass integrative Maßnahmen und Wohnraumpolitik aufeinander abgestimmt sind. Insbesondere die städtischen Ballungsräume stehen vor der Herausforderung, eine soziale und ethnische Ausgewogenheit der Bevölkerung vor Ort zu gewährleisten, um mögliche integrationshindernde Faktoren zu minimieren" (BMEIA und EXPERTENRAT FÜR INTEGRATION 2017, p. 57). Die Gemeinden nehmen die Rolle wichtiger Akteure im Integrationsprozess ein „welche insbesondere durch die Stärkung der politischen Verantwortung in den Gemeinden, etwa durch die Schaffung von IntegrationsgemeinderätInnen, ausgebaut werden soll.“ (vgl. ebd.). Infolge der kleingemeindlich geprägten Struktur in vielen Regionen Österreichs stellen sich die Aufgaben der Wohnintegration der Geflüchteten zwar primär in Großstädten, allen voran in der Agglomeration Wien sowie in den Zentralräumen von Linz und Graz, die „refugee crisis“ hat aber erstmals auch viele kleinere Gemeinden mit den vielfältigen Herausforderungen der Wohnintegration konfrontiert (ÖIF 2016; EuRopÄIsCHes Forum ALPBACH 2015) und umsetzungsorientierte Ansätze zur Integration von Geflüchteten stimuliert. ${ }^{105}$ In ländlichen Kommunen unterscheiden sich die Situationen vor Ort sowie die Entscheidungs- und Handlungsoptionen signifikant von jenen in den urbanen Agglomerationen (vgl. GRUBER 2013).

Wien ist im Vergleich zu anderen europäischen Metropolen durch ein niedrigeres Ausmaß an ethnischer Wohnsegregation charakterisiert (HATz et al. 2015; Goodson 2006; PhILlIPs 2006). Allerdings existieren für Österreichs Städte nur wenige Untersuchungen, welche die Wohnintegration Geflüchteter untersuchen (vgl. UNHCR 2013; AIgNER 2016, 2018). Einigkeit besteht darüber, dass der Wegzug aus dem Grundversorgungsquartier, oft in ein anderes Bundesland, viele Geflüchtete vor beträchtliche Probleme stellt. Die wichtigsten Einflussfaktoren der Wohnintegration haben u.a. Neuwirth (2005), CARItas Linz (2008) und Fetz (2011a) analysiert. Die Verflechtung der Teilbereiche struktureller Integration wird von KUCERA (2001), SCHEIBER (2007) und Fetz (2011a) bestätigt. Volf (2001), Schilcher (2009) und UNHCR (2015, p. 31) betonen die vielfältigen negativen Auswirkungen prekärer Wohnverhältnisse von Asylberechtigten. In österreichischen Städten erfolgt die Wohnintegration von

105 Vgl. <http://raum4refugees.at/wp-content/uploads/2015/11/Alpbach_Handbuch_Buerger meisterInnen_Asylquartiere.pdf $>$ 
Immigrant/inn/en nicht entlang ethnischer Segregationslinien, sondern spiegelt in erster Linie deren benachteiligte Platzierung auf dem Arbeitsmarkt und als Konsequenz die schwächere Einkommenssituation wider. Asylberechtigte sind denselben Marktmechanismen ausgesetzt wie andere Wohnungssuchende mit Migrationshintergrund. Im Vergleich zur restlichen Bevölkerung (auch jener mit Migrationshintergrund) sind Geflüchtete aber mit zusätzlichen Hürden (z.B. Xenophobie bzw. Islamophobie, kaum finanzielle Ressourcen, Unkenntnis des Wohnungsmarktes, fehlende Erfahrungen mit Mietverträgen) beim Zugang zu leistbarem Wohnraum konfrontiert. Überdies sind nur beschränkte kommunale Handlungsoptionen vorhanden. Antisegregationsstrategien bestehen vor allem in der Verfügbarkeit eines Stocks an Gemeindewohnungen, über deren Vergabe stadtnahe Serviceeinrichtungen der Wohnungszuteilung (z.B. Wiener Wohnen) entscheiden. Eine wichtige Rolle spielen die unterschiedlichen Wohnbauträger, die sich der Gemeinnützigkeit (z.B. Sozialbau) verpflichtet haben.

\subsubsection{Analyse der Interviews}

\subsubsection{Wohn- und Haushaltsformen}

Die Wohn- und Haushaltsstrukturen sind vielfältig und spiegeln die aktuelle Wohnraumknappheit und das hohe Mietpreisniveau wider, welche dem Wohnungsmarkt vor allem in Wien ihren Stempel aufdrücken. Bezüglich des Rechtsstatus dominieren Mietwohnungen besonders bei den Interviewten mit kürzerer Aufenthaltsdauer. Länger in Österreich lebende Afghan/inn/en bewohnen auch Wohnungen gemeinnütziger Bauträger, der Gemeinden oder des Eigentumssegments. Ein Teil der Befragten wohnt in Kleinwohnungen alleine, mit dem/der Ehepartner/in oder in größeren Wohnungen im Verband einer Zweigenerationenfamilie. Wohngemeinschaften (meist Freunde bzw. Bekannte) sind eine häufig gewählte Strategie zur Abfederung hoher Mietpreise. Im Falle rezent Zugewanderter ist der Überbelag in einigen Fällen extrem.

„Ich wohne mit einem Freund von mir, den ich seit fünf bis sechs Jahren kenne, ... in einer Wohngemeinschaft sozusagen. “ (I16, männlich, *1997, seit 2012 in Ö.)

„Ich wohne derzeit in einer WG. Eine Wohnung mit drei Zimmern, in der wir zu elft wohnen und wir sind wirklich sehr viel. Früher war es geplant, dass jene mit der weißen Karte von der Caritas eine Wohnung bekommen. Mit 350 Euro kann man ja keine Wohnung für drei bis vier Personen mieten. Aber zu elft in einer Wohnung ist es sehr schwierig. " (I54, männlich, *1996, seit 2015 in Ö.)

\subsubsection{Verflechtungen der Wohnintegration mit anderen Bereichen struktureller Integration}

Der Druck zur Aufnahme jeder sich bietenden Beschäftigung wird infolge der Finanzierung der Wohnkosten erhöht, obwohl daraus für die Individuen langfristige kompetitive Nachteile auf dem Arbeitsmarkt resultieren. Die wechselseitigen Abhängigkeiten von Wohn-, Bildungs- und Arbeitsintegration sind vielfältig: 
„Eigentlich hauptsächlich um einen Job zu finden, weil ohne Job kannst du auch keine Wohnung finanzieren. " (E4)

„, Wenn ich an einem Ort gut leben kann, gut studieren, gut arbeiten, dann ist es für mich sehr gut. ... Weil ich dort meine Arbeit verloren habe, bin ich dann nach Linz gezogen. Dann habe ich wieder eine Arbeit in der Steiermark gefunden und habe in Linz gekündigt. Dann habe ich im Jahre 2008 wieder eine Stelle in Wien gefunden und bin hierhergezogen. Aber ich bin mit all meinen Wohnorten zufrieden. " (I2, männlich, *1998, seit 2015 in Ö.)

„Aber jetzt ist nicht so möglich zu lernen. Muss ich zuerst arbeiten ein bisschen und dann zuerst noch die Wohnung suchen. Kann ich nicht so lang ... bleiben, weil noch ein Monat und dann kommt die Frau von ihm, und dann muss ich raus. Und dann muss ich eine Wohnung suchen. Aber so zwei Wochen ich suche die Wohnung, aber leider zu teuer. Kostet so viel. “ (I25, männlich, *1997, seit 2014 in Ö.)

Infolge des Zuzugs aus den Bundesländern sowie der Wohnungsknappheit auf dem urbanen Wohnungsmarkt verkomplizieren sich Arbeits- und Wohnungsmarktintegration in Wien, während diese Prozesse in kleineren Gemeinden meist problemloser verlaufen. Expert/inn/en betonen auch die Divergenz zwischen Wohnungs- und Arbeitsmarktperspektiven, denn kostengünstiger Wohnraum ist selten dort vorhanden, wo es Arbeitsplätze gibt:

„Noch dazu in Wien, hast du halt den Zuzug aus den anderen Bundesländern. Weil die die Mindestsicherung kürzen, hast du noch zusätzlichen Druck auf den Arbeitsmarkt; auf den Wohnungsmarkt, natürlich auch bis zu einem gewissen Grad,... “(E5)

„Ja, also die Community spielt schon die Hauptrolle, weil es ist halt auch so, das Überleben, die Wohnung finden und so, das ist auch auf dem Land, ... also, dass man in einem Gebiet ist, wo die Wohnungen auch erschwinglich sind und wo es Jobs gibt, das ist jetzt eher selten. " (E2)

Im Kontext der Integrationsherausforderungen (z.B. Erlernen des Deutschen, Absolvierung von Ausbildungen, Arbeitssuche) stellt es für viele eine zusätzliche Belastung dar, sich eigeninitiativ um eine Wohnmöglichkeit kümmern zu müssen. Dem Druck, der auf den rezent Geflüchteten lastet, stellen bereits länger in Österreich lebende Afghan/inn/en ihre früheren Erfahrungen bzw. jene ihrer Eltern, die in früheren Zuwanderungswellen nach Österreich gelangten, gegenüber. Damals war der Druck auf dem Wohnungsmarkt noch geringer, NGOs übernahmen effizient für die zahlenmäßig kleinen Gruppen Geflüchteter die Wohnungsvermittlung.

„Das ist mal die größte, also eine große Herausforderung mit den Terminen und so, die sie am Anfang erleben. Und zweitens, wenn sie kommen, ... sie haben eine Wohnung, die total problematisch ist. Sie haben zwar eine Wohnung, aber sie wissen, in zwei Jahren müssen sie raus sein, ... Wohnung ist total kaputt, haben 
extrem hohe Miete gezahlt, Provision, Kaution, hat parallel irgendeine Firma, die ihnen das Geld geliehen hat, und so weiter. Also total in einem Markt ... Sie kennen die Sprache nicht, gesundheitliche Probleme, psychosomatische Schwierigkeiten entstehen dann auch, sprachliche Probleme, Barrieren. Und dann vielleicht Verfahren nebenbei, das nicht richtig abgeschlossen ist ... Dann kommt der Druck, sie müssen Deutsch lernen, arbeiten. Und sie können nicht, wenn sie ein Zimmer haben und fünf Kinder haben, dass sie im Deutschkurs lernen. Dann können sie nicht nach Hause gehen, in einer Ecke sitzen und die Sprache weiterlernen. Es sind so viele Hürden am Anfang, dass es parallel nicht geht. Und ich sag 'immer: Früher war's ja so, ... da hat es weniger Projekte gegeben, so für die ganzen Sprachkurse oder so, aber da hat man sicherere Systeme gehabt. Man hat zum Beispiel eine Wohnung bekommen über Caritas oder über so, die Möglichkeit gab’s. ... Das heißt, die Grundbedürfnisse ... waren eigentlich sehr gut geregelt. “(E8)

\subsubsection{Wohnerwartungen}

Von Expert/inn/enseite werden die Erwartungen in einem Kontinuum von relativ „,normal, bieder“ bis als „,zu hoch“ klassifiziert. Seitens einiger Respondent/inn/en bestehen zwar Hoffnungen auf große, leistbare Wohnungen, viele äußern sich aber auch gar nicht über ihre Wohnerwartungen. In manchen Fällen erwarten sich die Klient/inn/en von den Betreuer/inne/n in den NGOs, dass die ihnen auch eine Wohnung verschaffen. In solchen Fällen müssen Erwartungen oft enttäuscht werden.

„Ich würde gerne eine schöne große Wohnung haben, für welche ich auch die Miete problemlos zahlen kann. "(I57, weiblich, *1966, seit 2015 in Ö.)

„Also wenn, dann haben die vielleicht zu große Erwartungen. Vielleicht hoffen sie, dass ... man ihnen was verschafft und vielleicht gegen Ende zu merken sie, dass diese Erwartungen nicht erfüllt werden. ... Klienten - das betrifft jetzt nicht nur die Afghanen - ... sie glauben halt, dass der Therapeut oder irgendwie da mehr verschaffen könnte oder durch bestimmte Schreiben oder Papiere ... “ (E9)

„Negative [Erfahrungen] habe ich sehr viel gemacht. Ich hatte in Afghanistan eine höhere Position, und dass wir hier keinen Bescheid haben und die Wohnung klein ist, sind alles Negative. Ich dachte, wenn ich in Österreich bin, sind meine Probleme gelöst, aber hier sind sie noch mehr geworden. " (I59, weiblich, *1999, seit 2016 in Ö.)

\subsubsection{Wohnungssuche}

Probleme und Herausforderungen

Nach dem Asylbescheid bleiben nur vier Monate bis die Asylunterkunft verlassen und eine eigene Wohnung gefunden werden muss. Die Mehrheit berichtet von komplexen Schwierigkeiten, manche haben diese krisenhafte Phase mittels Informationsdiffusion und Support in sozialen Netzwerken leichter bewältigt. Rezent ist anzunehmen, dass 
die Wohnungssuche über Social Media immer wichtiger wird, diese wird allerdings im Sample selten erwähnt. Überhöhte Mieten, Überbelag (oft bei Wohngemeinschaften, aber auch in Familienhaushalten) und aus mietrechtlicher Perspektive problematische Untermietverhältnisse drücken der Wohnsituation vieler Afghan/inn/en den Stempel auf. NGOs sowie Ehrenamtliche spielen eine wichtige Rolle bei der Wohnintegration. Vor allem in kleineren Gemeinden übernehmen sie die Wohnungsvermittlung. Einigkeit besteht darüber, dass es in Wien am schwierigsten ist Fuß zu fassen. Als Positivum heben Befragte hervor, dass dafür in Wien vielfältigere Hilfestellungen geboten werden. Manchmal bestehen krasse Ausbeutungsverhältnisse durch Vermieter/innen (nicht selten Angehörige migrantischer Communities), die später zugewanderte Geflüchtete ökonomisch ausbeuten, indem sie Wohnungen ohne Mietvertrag und zu überhöhten Mieten bereitstellen und das mangelnde Wissen über die österreichische Rechtslage ausnutzen.

Trotz der prekären Wohnverhältnisse vieler Geflüchteter bildet Obdachlosigkeit im Sample die Ausnahme. Vor allem junge Männer sind hinsichtlich ihrer Wohnansprüche flexibel und passen sich den Gegebenheiten (z.B. mit Wohngemeinschaften) an. Allerdings schützt auch das Wohnen in einer WG nicht immer vor hohen Mietkosten und die Stabilität dieser Wohnform ist in vielen Fällen gering.

„,Wohnen: Natürlich, in Wien ist das Wohnen für alle - es ist vollkommen egal, Asylberechtigte oder Asyl ... Immigrant oder halt , echte Österreicher “ - also eine Wohnung zu finden ist natürlich in Wien schwer ... " (E4)

„In Wien ist allgemein sehr schwer. Viele wandern dann aus den Bundesländern selbständig nach Wien, weil entweder denken sie, dieser Bezug zu den Communities ist da, und zweitens denken sie, in Wien ist vieles vielleicht viel leichter. Und dann sind sie ziemlich unglücklich, weil sie sagen: ,Ah, da waren wir-keine Ahnung - in einem Bundesland, und da ging es uns ja sehr gut. Wir waren bei den Nachbarn auch wohl gut aufgenommen und wir wollten unbedingt nach Wien. Oder die Kinder wollen nach Wien. ' Und das ist dann eben schwer. Und parallel hat sich ja natürlich auch in dem Wohnungsmarkt so private Bereich entwickelt, wo man überhaupt keine Transparenz hat, keinen Überblick hat. Da sind auf dem Privatmarkt Wohnungen, wo die Flüchtlinge wohnen, teilweise ganz in schlechten Zuständen ... extrem überteuert. Dann haben sie kurze Verträge. Also die Wohnungssituation von vielen, vielen, die teilweise auch zu uns kommen, ist so schlecht ... Wir können's nicht vorstellen. Und da ist die Möglichkeit, dass wenn man einen Status hat, ein anerkannter Konventionsflüchtling ist zum Beispiel in Wien und zwei Jahre an einer Adresse gewohnt hat und eigentlich der Mietvertrag nicht verlängert wird. Also da kommt Wiener Wohnen sehr entgegen. Auch die Wohnungskommission die schauen sich die Fälle an. Aber wenn ich da vergleiche, wie viele zu uns kommen und Schwierigkeiten haben, ist das auch dann schwer. Nicht jeder bekommt eine Wohnung und viele bekommen dann Angst, weil es ist ja immer so, dass wenn der Mietvertrag nicht verlängert wird, das wird dann alles gerichtlich entschieden. Und viele Menschen haben dann diese Angst vor dem 
Gericht und können dann auch nicht warten, suchen sich wieder eine überteuerte Wohnung aus und sagen, Lieber will ich jetzt den Streit nachgeben.' Aber die Wohnungssituation ist derzeit ganz schlecht. In Wien zumindest. In den Bundesländern habe ich keine Erfahrung. ... Und die Flüchtlinge schon gar nicht. ... Die sind teilweise extrem verschuldet, auch so bei privaten Leuten, nur weil sie Kaution und Provision für die Wohnungen zahlen. " (E8)

„,... also Wohnungen das ist jetzt nicht nur für die Afghanen ein Riesenproblem, sondern für alle .... Ja, es gibt einfach nicht genug Wohnungen oder Angebote. Dadurch sind die Preise hoch und dazu wird halt noch ziemlich viel Schindluder getrieben von halt so Leuten, die viele Wohnungen, also die sieben, acht Leute in die Wohnung packen und dann von jedem 200 Euro kassieren oder so. Bei Asylberechtigen und Asylwerbern. Das betrifft durch die Bank alle. “ (E5)

„, Und leider hören wir immer wieder, dass einfach bestimmte Menschen daraus Profit schlagen wie Immobilienmakler. Dass diese Leute sofort diese Wohnung mit kleiner Provision, kleine Provision 500 Euro, was für diese Person eigentlich extrem viel Geld ist. Und diese Wohnung - müssen Sie auch wissen - 500 Euro kostet. 25m², 500 Euro für einen Asylwerber, Asylberechtigten, der am Anfang keinen Job hat, ist das extrem viel. Aber genau diese Probleme gibt es viele. ... Natürlich, es gibt viel zu wenige Privatwohnungen ... oder an Gruppen Wohnungen vermieten, obwohl er überhaupt kein Eigentümer ist. Die Person hat selber die Wohnung gemietet und untervermietet an fünf Personen. Eine kleine Wohnung, Einzimmerwohnung und fünf Personen leben da drinnen, kassiert. Und auf einmal Eigentümer kommt darauf und sagt: , Sofort ausziehen!' Und die stehen auf einmal auf der Straße, ohne gar nichts, ohne Rechte ...

„.... ich meine, bei den afghanischen jungen Männern ist zumindest ... die Möglichkeit, irgendwo unterzuschlupfen, wobei natürlich aber möglicherweise sich dieser halbobdachlose Zustand hinauszögert dadurch. ... die jungen Afghanen lösen das halt meistens so, dass sie dann in größeren Gruppen in mittelgroßen Wohnungen wohnen, sehr oft auch da Opfer von diversen Abhängigkeits- und Ausbeutungsverhältnissen werden. “ (E2)

„,Wegen einer Wohnung also, ich habe zuerst bei Diakonie um Hilfe gesucht. Die haben mir gesagt, die könnten auch nicht viel tun und haben mir aber ein paar Internetseiten gezeigt und habe dann durch Facebook eine WG gefunden. Dort bin ich dann hingegangen und habe mich vorgestellt. " (I62, männlich, *1994, seit 2015 in Ö.)

„Nachdem ich 18 wurde, musste ich aus dem Heim in 1120 Wien ausziehen und bin dann zu dem Heim im Westbahnhof, ein Heim von Caritas, gegangen und habe dort für zwei Monaten gewohnt. Ich war für 15 Tage ohne Wohnung. Ich hatte weder Wohnung noch Geld - nichts. Es waren Tage, an denen ich an der Donau geschlafen habe, aber bei meinem Bruder bin ich nicht geblieben, weil es sehr ... 
Menschen waren. Seitdem mein Bruder einen Job hat, bin ich zu ihm umgezogen. “ (I56, männlich, *1999, seit 2015 in Ö.)

Relevanz der Unterstützung durch soziale Netzwerke

Signifikant häufig wird die Wichtigkeit eines funktionierenden sozialen Netzwerks als Hilfestellung für die Wohnintegration betont. Hierbei kann es sich um mono- oder gemischtethnische soziale Netzwerke handeln. Diese kompensieren Informationsdefizite über den Wohnungsmarkt, finanzielle und sprachliche Probleme sowie Zugangshürden aufgrund von Xenophobie gegenüber Afghan/inn/en. Die Vermittlung von Wohnungen erfolgt entweder über Landsleute aus Afghanistan oder mithilfe von Österreicher/inne/n. Die Palette letzterer reicht hier von Lehrer/inne/n in Deutschkursen, über Mitarbeiter/innen von NGOs bis zu Ehrenamtlichen. Support von Personen mit Migrationshintergrund außerhalb der afghanischen Community wird selten genannt. Bewusst ziehen viele in Form einer „chain migration“ (BLOCH 2002; HAUG 2008) auch dorthin, wo bereits Familienangehörige leben, um einander sozial und ökonomisch absichern zu können.

ich gehe davon aus, dass so Freundschaften oder Bekanntschaften, die in Wien sind, dass das natürlich eine Rolle spielt. ... Ja, also ich glaub', dass das auf jeden Fall die Leute, wenn sie schon persönliche Verknüpfungen irgendwohin haben, ... das einfacher macht, zu sagen: ,Ja, ich zieh ' da hin. '... Es ist so, dass Leute im Asylverfahren ja teilweise das Bundesland verlassen und nach Wien ziehen und wenn einer fragt: ,Ok, warum?' ,Ja, ich hab 'Freunde hier. '... Das hört man oft. “ (E5)

„... nach dem Positivbescheid wir mussten von Asylheim weg, wir haben vier Monate gehabt .... und eine private Wohnung finden. Und eine Lehrerin von der Schule hat mir gesagt, dass sie kann mir helfen ... Sie hat gesagt: , Wir haben eine Wohnung, dort lebt eine Krankenschwester und in zwei Monaten sie muss weg und du kannst die Wohnung schauen und kommen. 'Dann wir sind in diese Wohnung umgezogen. Und es ist eine schöne Wohnung mit einem großen Garten, ich finde österreichische Leute sehr nett. “ (I26, männlich, *1974, seit 2013 in Ö.)

„,... Deswegen waren wir gezwungen, uns eine Wohnung zu suchen, und diese Person [Anm.: Afghane], mit der ich jetzt wohne, hat mir geholfen, diese Wohnung zu finden. Am Anfang hat diese Person uns sehr geholfen, weil wir auch die Sprache nicht verstanden haben. Jetzt arbeitet diese Person mit den Flüchtlingen beim Roten Kreuz. “ (I54, männlich, *1996, seit 2015 in Ö.)

\section{Konfrontation mit Xenophobie}

Die Community aus Afghanistan wird mit einem zusätzlichen, aus der medialen Berichterstattung resultierenden Problem konfrontiert, welches während des Erhebungszeitraums zugenommen hat: Infolge einer Reihe von Straftaten hat das Image der gesamten Community gelitten, sodass wenige Wohnungseigentümer/innen an Afghan/ inn/en vermieten möchten. Diese Stigmatisierung hat die Problematik der Wohnungs- 
marktintegration verschärft. Afghan/inn/en werden in der Folge umso leichter Opfer von Kriminellen, die nicht rechtskräftige Untermietverhältnisse zu überhöhten Mietpreisen offerieren:

„Bei der Wohnungssuche haben die Afghanen momentan durch diese bestimmten Kriminalfälle und Medienberichte keinen guten Stand, wenn sie eine Wohnung und Arbeit suchen. Die Leute haben keine gute Meinung von Afghanen. ... Es ist nicht so, dass alle so sind. Es gibt Leute, die Wohnungen vermieten usw. Aber es ist schwierig, dass sie eine Wohnung finden. Die Wohnungsknappheit muss man auch in Betracht ziehen, das hat nicht unbedingt mit Migranten zu tun. " (E3)

„Immobilienbüros geben da keine Wohnungen. Es ist durch die ganzen negativen Sachen, was in den Zeitungen gestanden sind. Also Afghanen und Tschetschenen haben sehr schlechte Karten bei dieser Sache. Auch bei der Arbeit ist es genau dasselbe. Man nimmt irgendeinen anderen, wenn zwei Leute kommen, der eine ist ein Afghane und der andere ist von irgendwo anders, dann nimmt man den und lasst den Afghanen stehen, weil die sagen, der streitet und solche Sachen. Und bei der Wohnung genau dasselbe. ... Wohnungen sind da, aber sie bekommen die nicht. Weil da eigentlich dieses negative Image da ist. .... Nur in Wien ist auch jetzt schwierig, dieses Problem, was Wohnen betrifft. ... Ich habe selber am Anfang jemanden angerufen, alles fertiggemacht. Er hat gesagt: ,Ok, kommen Sie morgen. ' Ganz zum Schluss fragt sie dann: [lacht] ,Sie sprechen ja so gut Deutsch. Von welcher Nationalität sind Sie?' Sage ich: ,Ich bin ein Afghane. 'Und sagt sie: ,Nein, tut mir leid, wir geben Ihnen die Wohnung nicht. 'Sage ich: , Warum nicht? Ich habe alles mit Ihnen ausgemacht. 'Sagt sie: , Nein, der Wohnungsinhaber hat eigentlich ausdrücklich das gesagt, dass wir den Afghanen keine Wohnungen geben. ' Und das ist schon ein Problem. " (E7)

\subsubsection{Räumliche Wohnpräferenzen und Binnenmigration}

In vielen EU-Staaten ist die Konzentration der Asylberechtigten auf die urbanen Agglomerationen - im Gegensatz zu der politisch und ökonomisch erwünschten „Gleichverteilung“ - eine vieldiskutierte Thematik (BIRGIER et al. 2016; DAHLBERG und VALEYATHEEPILLAY 2018; THIELEMANN 2018). Medial wurden positive Beispiele erfolgreicher Integration der Geflüchteten in kleineren Gemeinden bzw. im ländlichen Raum der starken Tendenz zur Binnenmigration in die Großstädte gegenübergestellt (FABRY 2018; HAJEK 2017). Risiken der ausgeprägten „community formation“ bis zur potentiellen Etablierung von Parallelgesellschaften in urbanen Teilräumen werden daraus abgeleitet. Mit der Reduktion der Asylwerber/innenzahlen ist diese Diskussion wieder abgeflaut. Um die persönlichen Präferenzen abzuklären wurden die Afghan/inne/en nach den aus ihrer Perspektive festzustellenden Vor- und Nachteilen der beiden Raumtypen befragt. Das Ergebnis stellt eine Momentaufnahme der Wohnpräferenzen zum Erhebungszeitpunkt dar, denn eine längerfristige Analyse der räumlichen Integrationsverläufe war nicht möglich. Es konnten aber Rückschlüsse auf das hohe Ausmaß geplanter Wohnsitz- 
verlagerungen in die Agglomerationen gezogen werden. Ein Konnex zwischen urbanem Herkunftskontext im Ursprungsland und der Bevorzugung der vielfältigen Chancen des Stadtlebens ist auch in Österreich evident.

Das Gros der Befragten äußerte sich explizit dazu, wo sie längerfristig lieber leben wollten. Die meisten davon im urbanen Kontext. Die von den Interviewten aufgezählten Positiva und Negative der räumlichen Kontexte Stadt und Land sind vielfältig. Die Vorstellung, dass die berufliche und die Wohnintegration in Wien leichter fallen könnte, tritt häufig auf. Dazu kommen weitere Faktoren, die für ein Wohnen in der Stadt sprechen: Herkunft aus dem urbanen Kontext im Ursprungsland, soziale Netzwerke zu Verwandten und Freunden, österreichische Partner/innen in der Stadt, die ethnische Community, religiöse Infrastruktur, Dichte des öffentlichen Personennahverkehrs, Zugang zu beruflicher Weiterbildung und Deutschkursen, Unterhaltungs- und Freizeitangebote, Wellbeing, geringere Xenophobie, das Fehlen von frei zugänglichem Public Space in Kleingemeinden, die Anonymität, geringere finanzielle Aufwendungen für die Lebensgestaltung. Die Möglichkeiten der spontanen Kontaktaufnahme werden ebenfalls als Pluspunkte der Großstadt angeführt. Vorhandene bzw. fehlende Kontaktfreudigkeit wird aber sehr unterschiedlich erlebt. Lebensqualitätskriterien wie Ruhe, naturbelassene Umwelt und gute Luft- sowie Umweltqualität sprechen für das Landleben. Eine Barriere bilden lokale dialektale Besonderheiten, die es den Geflüchteten erschweren, sprachlich $\mathrm{Fuß} \mathrm{zu}$ fassen. Soziale Isolation und daraus resultierende psychische Probleme werden je nach individueller Erfahrung sowohl bei der Bewertung der Vorteile des urbanen als auch ruralen Raumes ins Treffen geführt. Bezüglich der Tendenz zur Binnenmigration finden sich in den Interviews also sehr unterschiedliche Befunde. Diese reichen von einer sachlich argumentierten Ablehnung gegen eine der beiden räumlichen Kategorien und in Reaktion darauf vollzogene Wohnortwechsel bis zu sehr emotionalen Begründungen, weshalb das Land- oder Stadtleben bevorzugt wird. Aufgrund der Fülle an Material kann hier nur eine begrenzte Selektion wiedergegeben werden:

\section{Argumentationen für die Wohnintegration im ländlichen Raum:}

„,... wo es schon starke Initiativen gibt - ehrenamtliche -, die dann halt für die Leute irgendwelche Jobs und Wohnung checken. Und dann bleiben die Leute zum Teil dort. Aber auch nicht immer. Manchmal sind sie dann weg und dann sind die Ehrenamtlichen ganz enttäuscht und fühlen sich verraten von ihren Flüchtlingen. Aber das finde ich als Thema wahnsinnig interessant, diese Integrationen im ländlichen Raum, weil ich es für den ländlichen Raum für eine Chance auch halten würde, dass da mehr Diversität entsteht und einfach eine andere Dynamik reinkommt. ... Das wird immer unterschätzt, die Dynamik, die Migranten und Flüchtlinge haben, die Energien haben, die irgendwelche Pizzerien aufmachen. Irgendwo im Waldviertel hat gleich einer - der war dort untergebracht in Grundversorgung und kaum hat er Asyl gekriegt-, hat er schon eine Pizzeria aufgemacht und gleich zwei Angestellte, die auch dort waren. Also das funktioniert zum 
Teil eh. Aber es ist ein Prozess, der sehr am Laufen ist. Und wenn man dann in zehn Jahren sieht, was sich da entwickelt hat. "(E2)

„Es ist zurzeit ein bisschen, was Arbeit betrifft, glaube ich, außerhalb besser, nur viele wollen eigentlich da, damit die Community hier ein bisschen stärker ist, dass sie versuchen, hier zu arbeiten. " (E7)

„In einer kleinen Stadt, weil die Erwartungen dort klein sind. Es ist auch leichter dich in so einer Gesellschaft zu integrieren. In der Hauptstadt ist sehr viel los, und da die öffentlichen Verkehrsmittel viel sind, wollen viele hierherziehen. Aber in einer kleinen Stadt kann man sich besser entwickeln. ... In einer kleinen Stadt, weil es weniger Migrant/inn/en gibt. Die Menschen reden selbst mit dir und nehmen Kontakt auf. Zum Beispiel ich selbst: In dem einen Jahr im Dorf sind die Menschen immer zu mir gekommen und haben sich mit mir unterhalten und mich gefragt, ob ich was brauche. Ich konnte mich in dem einen Jahr mit vielen Menschen unterhalten. Zum Beispiel, wenn ich Fußballspielen war und ich alleine war, haben sie mich gefragt, ob ich auch mitspielen möchte. Aber in Wien habe ich sowas noch nicht erlebt, weil hier sehr viele Migrant/inn/en leben und die Menschen haben keine gute Erfahrung mit ihnen. “ (I49, männlich, *1997, seit 2015 in Ö.)

„Ich habe in NÖ. gelebt, und es war besser dort. Es war ruhig und wenig Lärm. Nach Wien bin ich wegen der Arbeit umgezogen und ich bin mit dem Bezirk, in dem ich wohne, nicht zufrieden. ... Ich mag NÖ. sehr, weil dort es sehr ruhig ist und sehr wenig Lärm. " (I51, männlich, *1993, seit 2010 in Ö.)

Argumentationen für die Wohnintegration im urbanen Raum:

„, Und die haben Zeit in diesen zwei Jahren die Erfahrungen von den anderen zu sammeln und sagen: ,Ok, doch in Wien hab'ich mehr Möglichkeiten. Doch in Wien bekomme ich mehr Chancen und Möglichkeiten. 'Alleine Deutschkurs, spezifische Deutschkurse im Fachbereich. Also wenn ich halt, weiß ich nicht, ein Maschinenschlosser war, bekomme ich durch AMS einen Deutschkurs in diesem Fachbereich, damit ich schneller zu einem Job komme. Und das ist leider in Bundesländern nicht der Fall. “ (E4)

„Also viele wollen direkt nach Wien kommen, ... weil sich in Wien sehr viel tut, politisch auch sehr viel tut. Auch gibt es viele Projekte vom Arbeitsmarktbereich. Diese vielen Deutschkurse, und das ist so zentral und in Wien können sie sich sehr gut bewegen. Also in den Bundesländern bei vielen, die Analphabeten sind und am Land sind, die Herausforderung ist mal, mit den öffentlichen Verkehrsmitteln irgendwo anzukommen, mit dem Zug oder Bus. Aber in Wien ist das dann so gut mit den Verbindungen, dass sich dann jeder in Wien gut fühlt. Und zweitens, die Stadt Wien macht ja so viel. Sie arbeiten auf der Ebene von Communities zusammen. ... also genauso in NGO-Bereichen wird so vieles angeboten, dass die Menschen das Gefühl haben, die haben so viele Informationen und dass sie da weiterkommen. "(E8) 
„Die meisten wollen in die Stadt gehen. ... Weil es in der Stadt Arbeitsmöglichkeit gibt. Weil es in der Stadt schnelle Kommunikationsmöglichkeit gibt mit ihren eigenen Landsleuten, weil sie in der Stadt eine Wohnung finden können, leichter finden können. ... die meisten wollen nach Wien und das hat mehrere Hintergründe. Zum Beispiel die Jugendlichen sind als Flüchtlinge gekommen von Iran, Pakistan, sie haben immer in den Städten gelebt. Und sie waren auf der Flucht immer in den Städten. ... Das Problem ist, das Leben auf Land ist für sie nicht attraktiv, sondern langweilig. Sie werden irgendwo auf einen Berg, in ein Tal ..., da gibt es kein Einkaufszentrum. Also für die noch nicht Anerkannten, keinen Sprachkurs und dort schon von Anfang an haben sie eine Aversion dann gegen das Land und sie wollen in die Stadt ziehen. " (E3)

„Ich habe bei Wiener Neustadt in einem kleinen Dorf gewohnt, in dem nur alte Menschen wohnten. Die Jungen sind in die Stadt zum Arbeiten und Studieren umgezogen. Der Ort war überhaupt nicht gut für meine Psyche. Ich bin nach Wien umgezogen, und es gefällt mir sehr hier. Ist eine große Stadt, mehr Menschen, habe viele Freunde ... “ (I49, männlich, *1997, seit 2015 in Ö.)

„,Wien hat viele Möglichkeiten. ... Also Wien ist einfach so die kleine perfekte Stadt zum Leben für Familien, für mich persönlich auch ... weil man hat echt alles hier. “ (I14, weiblich, *1996, seit 2001 in Ö.)

„Hier gibt es viele Leute und viele Geschäfte von Türkisch, Halal und so. ... Von unserer Tradition und Kultur gibt es viel Möglichkeiten und viele Leute. Und viel Verkehr, das ist so gut. Aber als ich am Land war, ich bin mit dem Fahrrad gefahren und mein Leben war in Gefahr. ... Stadt ist für mich sehr wichtig, und die Kinder können leicht zur Schule oder Gymnasium gehen und so leicht nach Hause kommen. Und man kann auch besser bis 10 oder 12 Uhr in der Nacht spazieren gehen oder Eis essen oder so ... aber am Land gibt es nicht diese Möglichkeit. “ (I20, weiblich, *1964, seit 2013 in Ö.)

Keine Präferenzen für einen Raumtypus bzw. ambivalente Bewertungen

Mitunter werden auch keine Präferenzen für das Leben in einem bestimmten räumlichen Kontext geäußert oder beiden Raumkategorien werden Vor- und Nachteile zugeschrieben.

„,Derzeit wohne ich im 19. Bezirk, aber es ist überall schön in Österreich, weil es Sicherheit gibt und niemanden geht was an, was die/der andere macht, und ich kann ganz in Ruhe meinen Sachen nachgehen. “ (I56, männlich, *1999, seit 2015 in Ö.)

„Ich denke immer, dass der genaue Ort für mich nicht so wichtig ist. Wenn ich an einem Ort gut leben kann, gut studieren, gut arbeiten, dann ist es für mich sehr gut. Als ich von meinem Heimatland hergekommen bin, war es für mich überhaupt kein Problem, ob ich Wien wohne oder in einer anderen Stadt .... " (I2, männlich, *1998, seit 2015 in Ö.) 
Binnenmigration - die Rolle der Mindestsicherung

In der politischen und medialen Diskussion ${ }^{106}$ der vergangenen Jahre wurde häufig ein Konnex zwischen der Höhe der Mindestsicherung und einer nach Wien orientierten Binnenmigration hergestellt. Trotz expliziter Nachfrage wurden hierzu nur wenige Statements abgegeben. Es finden sich jeweils zwei, welche den Konnex zwischen der Höhe der Bedarfsorientierten Mindestsicherung und der nach Wien orientierten Migration bestätigen bzw. als nicht relevant einstufen. Häufig spielt wohl eine Kombination von verschiedensten Faktoren eine Rolle (vgl. vorhergehende Zitate).

„I: Und denken Sie, dass diese bundeslandspezifischen Sozialleistungen da auch eine Rolle spielen? E: Ja, das sicherlich .... Nur das wird dann jetzt glaube ich bald eh eingeschränkt. Das ist schon eingeschränkt, da können sie nicht leicht hierherkommen. "(E7)

„,Und die bleiben nicht in anderen Städten, Orten und die werden nach Wien kommen, in andere Bundesländer [Städte] kommen. ... Viele sagen, es ist nur wegen dem Sozialgeld, das stimmt auch nicht meiner Meinung nach. Es ist [dass sie in die Städte gehen], weil sie nicht von der Ortschaft aufgenommen wurden, nicht akzeptiert wurden, nicht in diese Ortschaft zumindest [integriert wurden], ..."(E12)

„I: War es für Sie von Bedeutung, dass die Höhe der staatlichen Unterstützung oder die Mindestsicherung in den Bundesländern unterschiedlich hoch sind? ... B: Ihre Rolle ist sehr wichtig, aber auch nicht so viel. Aber man ist doch auf diese Unterstützung angewiesen, um die Miete zu zahlen und das Leben zu finanzieren, solang man nicht arbeiten darf, oder keinen Job hat. " (I47, männlich, *1996, seit 2015 in Ö.)

„Es ist egal, dass das Zuhause [Anm.: eines Geflüchteten] weit weg ist von Salzburg, wo ich arbeiten gehe, das ist mir wurscht, aber Hauptsache meine Familie und ich haben dort ein Zuhause gefunden ... Und obwohl die Mindestsicherung ist in Salzburg ganz, ganz wenig. " (E12)

\section{Die Relevanz der Lokalisation der afghanischen Community}

In der Diskussion um ethnische Wohnsegregation wird häufig die Formation von Parallelgesellschaften angesprochen. Es wurde daher nachgefragt, inwieweit die ethnische Community relevant für die Wohnortentscheidung ist. Es dominiert die Einstellung, dass ein konfliktfreies Zusammenleben mit allen Herkunftsgruppen angestrebt wird, die Zusammensetzung der Nachbarschaft aber gleichgültig ist. Aus den Expertenaussagen ist die Wichtigkeit sowohl von Kontakten mit der eigenen ethnischen Community als auch mit der Mehrheitsgesellschaft abzuleiten. Die meisten Afghan/inn/ en bewerten das unterstützende Netzwerk als wesentliches Positivum der Community. Als negativ wird die rigide soziale Kontrolle empfunden.

106 Vgl. dazu Müller 2016; WinRoither und ThalHammer 2017. 
„,Viele wandern dann aus den Bundesländern selbständig nach Wien, weil entweder denken sie, dieser Bezug zu den Communities ist da .... "(E8)

„Montagabend war ich bei einer Hochzeit, afghanische Hochzeit, und da hab" ich einen Herrn getroffen, der bei Rohrbach gelebt hat. ... Und es war der einzige Familie dort in diesem Dorf. ... deswegen waren sie die Superstars, also alle kannten die Familie. ... Also alle waren glücklich eigentlich, bis auf die Frau, weil die Frau sich dort alleine gefühlt hat. Und als sie nach Wien gekommen sind, jetzt sind sie plötzlich alle glücklich. Warum? Weil sie in Wien sind. ... Das ist eigentlich für die Integration denke ich, dass es gut ist, wenn man mit den Einheimischen viel Kontakt hat und viel zu tun hat als nur in der Gesellschaft in der eigenen Community, ... ich persönlich meine, es ist beides wichtig. Sowohl der Kontakt zu der Aufnahmegesellschaft oder Österreichern, aber auch Kontakt zu der eigenen Community, weil hier braucht man auch ein bisschen soziale Kontakte, ... Kontakte aus der Heimat. Auch für die Anerkennung, weil dann weiß man: ,Ok, ich bin nicht hier alleine, sondern es gibt auch andere, die was gemacht haben. 'Aber auch als Rollenbild, ,role models', also zum Beispiel, ... bin neu, Flüchtling, komme nach Wien, dann plötzlich treffe ich einen Afghanen, der bei diesem Amt oder dort als Arzt arbeitet oder hier arbeitet und so. Dann plötzlich werde ich auch motiviert und sagen: ,Ok, was hast du gemacht? Und wie hast du es geschafft?'Deswegen ist beides aus meiner Sicht wichtig. “ (E6)

„,Mir ist es gleichgültig, ob es dort Moscheen gibt oder nicht oder ob es dort Afghanen gibt oder nicht. " (I57, weiblich, *1966, seit 2015 in Ö.)

Dass integrationsorientierte Familien sogar Wohnortwechsel vollziehen, um nicht in einem Umfeld zu leben, in welchem die afghanische Community zahlreich ist, belegt folgendes Statement:

„Es war im 23. Bezirk, aber es war nicht so schlecht. Weil es war besser, dass wir weggekommen sind ... vom 11. Bezirk, weil ... es waren sehr viele afghanische Familien. Und wenn die Kinder alle draußen waren, haben sie nur in ihrer Sprache geredet. So war es für uns besser. ... Ich glaube, dadurch haben wir dann schneller Deutsch gelernt. Wir mussten dann reden. "(I15, weiblich, *1993, seit 2001 in Ö.)

\subsubsection{Wohnnachbarschaft: Zwischen Xenophobie, Akzeptanz und Zugehörigkeit}

Wenn es Afghan/inn/en gelungen ist, eine Wohnung $\mathrm{zu}$ finden und auch im Falle bereits länger in Österreich lebender Personen wird vereinzelt über xenophob motivierte Probleme mit Nachbar/inne/n berichtet. Negativen Erfahrungen stehen auch positive Beziehungen der Befragten mit österreichischen Nachbar/inne/n gegenüber. Offensichtlich ist das gegenseitige Kennenlernen im Rahmen der Wohnnachbarschaft imstande, anfängliche Vorurteile zu beseitigen.

„Ja, das war im 23., das war auch ein Grund für meine Mama wegzuziehen. Unsere Nachbarn ... die Frau hat oft meine Mama beschimpft, grundlos angegangen ... es 
war eher rassistisch. Das war auch ein Grund, weil sie hat meine Mama ziemlich genervt mit ihrer Art. Immer wenn sie meine Mama allein erwischt hat, sozusagen, hat sie begonnen mit ihr zu schimpfen .... “ (I15, weiblich, *1993, seit 2001 in Ö.)

„, Wir haben einen Nachbarn gehabt, wie ich ganz frisch in die Wohnung eingezogen war mit meinem Mann. Ich hab 'immer, Hallo ' gesagt, die haben nie geantwortet. ... Und dann erst wie sie ganz alt war und ihre Mann ist gestorben, ihre Tochter war weg, ganz alt. Eines Tages ist zu uns gekommen: ,Können Sie mir helfen?'“ (I36, weiblich, seit 1976 in Ö.)

\subsection{Arbeitsmarkt und Beruf}

\subsubsection{Empirischer Forschungsstand}

„Labour market integration is an important indicator of short- and long-term refugee integration“ (WAXMAN 2001, p. 473), wobei auf Basis des State of the Art der Forschung feststeht, dass, ,employment and self-sufficiency have been called the 'greatest challenges' for refugees ...“(WHITE 2012 in UNHCR 2013, p. 3). Die Geflüchteten „... define economic outcomes such as employment as important to their own lives“ (VAltonen 1998, p. 42). Dies wird durch die empirischen Resultate der vorliegenden Erhebung bestätigt.

Die gesetzlichen Regelungen für die Arbeitsmarktintegration orientieren sich vor allem am Kriterium der Staatsbürgerschaft. Während für EU-Bürger/innen Freizügigkeit gilt, ist der Arbeitsmarktzugang für Drittstaatsangehörige eingeschränkt. MELANDER und Pichelmann (2015) sowie Manahl (2017) betonen die Wichtigkeit angemessener integrationspolitischer Maßnahmen in der Arbeitsmarktintegration, wobei Segmentation entlang ethnischer, religiöser oder sozialer Linien möglichst zu vermeiden sei.

$\mathrm{Zu}$ den empirisch belegten Fakten im Zusammenhang mit der Integration von Immigrant/inn/en in den Arbeitsmarkt gehören die geringeren Erwerbsquoten der Bevölkerung mit Migrationshintergrund (v.a. bei Frauen) (KopPEnBERG 2015; STATISTIK Austria 2017, p. 54), altersspezifische Unterschiede in der Erwerbsbeteiligung, Konzentrationen auf bestimmte Wirtschaftszweige bzw. auf untere Ebenen der Beschäftigungshierarchie sowie Dequalifikation. Diese Faktoren gelten verstärkt für Geflüchtete (vgl. Institut FÜR ArbeITSMARKT- Und BERUfSForschung 2015; BrÜCKER et al. 2017, 2018). Bildung ist eine der wichtigsten Determinanten der Arbeitsmarktintegration (BUBER-ENNSER et al. 2016), Erwerbserfahrung vor dem Zuzug, insbesondere jene mit Ausbildungsbezug, wirkt sich ebenfalls vorteilhaft auf die Arbeitsmarktintegration aus (Hosner et al. 2017, p. 86 f.). Fehlende Ausbildungsnachweise erschweren die Arbeitsmarktintegration jedoch erheblich (Verwiebe et al. 2019). Auch der starke Anstieg (in Österreich laut OECD um 15\% bis 2020) in der Gruppe der jungen, wenig qualifizierten Männer stellt ein Problem dar (HöLLER 2018). 
Die Kompetenzen der Arbeitsuchenden mit Fluchthintergrund und die Bedarfsstrukturen auf dem Arbeitsmarkt des Einwanderungslandes korrelieren nur selten (Bonin und RinNe 2017, p. 20). Es manifestiert sich ein „refugee gap“, der empirisch für viele nationale Arbeitsmärkte belegt werden konnte. Dieser besagt, dass sich Geflüchtete auf dem Arbeitsmarkt marginaler als andere Immigrant/inn/en positionieren (vgl. Portes und Stepick 1985; TAKeda 2000; WaXman 2001; Potocky-Tripodi 2003, 2004; Connor 2010; Hugo 2013). Das Phänomen wurde u.a. für Australien (Hugo 2011, 2013), Kanada (Yu et al. 2007), die USA (Cortes 2004; Connor 2010), Norwegen (AALANDSLID 2008) und Schweden (BEvelander 2011, 2009; Bevelander und Pendakur 2012) analysiert. Auf dem österreichischen Arbeitsmarkt geht die hohe Präsenz von Asylberechtigten und subsidiär Schutzberechtigten im niedrigqualifizierten Tätigkeitssegment einher mit deren ausgeprägter Dequalifikation (STADLER und Wiedenhofer-Galik 2011; UNHCR - Policy Development and Evaluation Service 2013, p. 33, 53; Koppenberg 2015, p. 79 f.; Hosner et al. 2017, p. 86 f.). Diese ist auf länderspezifische institutionelle Restriktionen sowie vielfältige, erschwerte Ausgangsbedingungen zurückzuführen (vgl. dazu BIFFL et al. 2012; PFEFFER und SKrIVANEK 2013; UNHCR - Policy Development and Evaluation Service 2013, p. 31 ff.; Höhne und Schulze Buschoff 2015; Aschauer und GANN 2017).

Die erstmals im August 2015 in Wien durchgeführten Kompetenzchecks des AMS belegten die Heterogenität des Qualifikationsprofils Geflüchteter sowie beträchtliche Anteile von Niedrigqualifizierten (vgl. Arbeitsmarktservice 2016). Koppenberg (2015, p. 77 f.) stellte des Weiteren auf der Grundlage der Auswertung des Ad-hocModuls „Arbeitsmarktsituation von Personen mit bzw. ohne Migrationshintergrund“ des Mikrozensus-Grundprogramms (2014) fest, dass von den Erwerbstätigen, welche Flucht oder Asyl als Hauptgrund ihrer Migration nach Österreich angaben, immerhin 25,8 \% der Kategorie der Hilfsarbeitskräfte hinzuzurechnen waren (vgl. STATISTIK Austria 2015, p. 44). Die entsprechenden Tätigkeiten werden als für Inländer/innen unattraktive „4D-Jobs“ (,dull, demeaning, dirty and dangerous“) bezeichnet. Asylberechtigte und subsidiär Schutzberechtigte arbeiten in der Anfangsphase ihrer beruflichen Integration auch überproportional häufig in atypischen Beschäftigungsverhältnissen (vgl. Hosner et al. 2017). Die hohe Konzentration in Hilfstätigkeiten ist vor allem in den Bundesländern typisch. In Wien ist die Verteilung auf die Berufsfelder heterogener (ebd., p. 86 f.), was die politisch häufig kritisierte Binnenmigration von Geflüchteten nach Wien rational rechtfertigt. Erfahrungswerte der befragten Expert/inn/en belegen (vgl. empirischer Teil), dass Asylberechtigte und subsidiär Schutzberechtigte Afghan/ inn/en in hohem Ausmaß unter ihrem Qualifikationsniveau beschäftigt sind (vgl. auch UNHCR 2013, p. 3). Für Wien gelangten RiESENFELDER et al. (2010, p. 124) zu einem Anteil von mehr als $40 \%$ befragter Personen mit internationalem Schutz in dequalifizierenden beruflichen Positionen. ScheIBER (2007, p. 157) belegte für Oberösterreich auf Basis eines kleinen Samples von Geflüchteten mit Universitätsabschlüssen, dass kein/e einzige/ $\mathrm{r}$ der Befragten einer der Ausbildung angemessenen Beschäftigung nachging. Von den Personen, die eine höhere Schule mit Matura (oder vergleichbarem Abschluss) 
im Herkunftsland absolviert hatten, übte nur eine Studierende eine Beschäftigung aus, die ihrem Bildungsniveau entsprach. Integrationsverläufe zwischen Integration und Exklusion im Falle syrischer und afghanischer Geflüchteter stellte KöNIG (2016) für den steirischen Arbeitsmarkt fest. Eine generell hohe Überqualifizierungsrate unter Migrant/ inn/en in Österreich konstatierte SMOLINER (2011, p. 96).

Bock-Schappelwein und Huber (2015) sowie Verwiebe et al. (2019) gelangten zu dem Resultat, dass Abschlüsse, die in Österreich erworben wurden, für die Arbeitsmarktintegration einen höheren Wert besitzen als solche aus dem Herkunftsland. Zusätzlich spielen individuelle Faktoren wie landesspezifische Kenntnisse sowie „soft skills“ (Offenheit, Kommunikationsbereitschaft, Kontaktfreudigkeit, Flexibilität, Einsatzbereitschaft, Frustrationstoleranz etc.) auf dem Weg zur erfolgreichen Arbeitssuche eine Rolle (vgl. HöHne und Koopmans 2010; LanceE 2012 u.a.). Von großer Relevanz sind überall soziale Kontakte zu Einheimischen, die die Stellensuche unterstützen (Aguilera 2002, p. 868; LANCEe 2012; Verwiebe et al. 2019). Diskriminierung aus ethnischen bzw. religiösen Gründen ist ein weltweites Problem (vgl. Hugo 2013 für Australien; Valtonen 2001 für den finnischen Arbeitsmarkt; KaAS und Manger 2012 für Deutschland sowie Blommaert 2013 für die Niederlande). Hosner et al. (2017, p. 86 f.) stellten fest, dass trotz des im Schnitt niedrigeren formalen Bildungsniveaus von Geflüchteten aus Afghanistan die Arbeitsmarktintegration nicht schlechter oder langsamer als bei anderen Gruppen (z.B. aus Tschetschenien oder dem Irak) funktioniert. Afghan/inn/en arbeiten allerdings am häufigsten als Hilfsarbeitskräfte und selten in hochqualifizierten Tätigkeiten. Daten des Arbeitsmarktservice (2019) belegen, dass $44 \%$ der Geflüchteten einer Kontrollgruppe einen Job gefunden hatten bzw. dass von den Geflüchteten mit gültigem Asylstatus/subsidiären Schutz, die ihren Asylstatus 2015 erhalten hatten und von 2015 bis Mitte 2016 beim AMS registriert wurden 61,7\% seit Jahresbeginn 2015 eine selbständige oder unselbständige Beschäftigung gefunden hatten, die länger als acht Tage gedauert hatte (ADDENDum 2019). Die Arbeitsmarktintegration von Geflüchteten in Österreich hat ein Team um Roland Verwiebe und Bernhard Kittel anhand von mehr als 1.000 Personen, die zwischen 2013 und 2017 ins Land kamen, untersucht (VerwieBE et al. 2019). Wie die Arbeitsmarktintegration von Geflüchteten funktioniert beschreiben auch JESTL und LEITNER (2019).

\subsubsection{Analyse der Interviews}

\subsubsection{Berufswünsche und Erwartungen}

Die Berufswünsche umfassen ein sehr breites Spektrum (von manuellen Professionen wie Maurer oder Bauhilfsarbeiter, über Büroberufe, Tätigkeiten im Sozialbereich oder im IT-Sektor, als Einzelhandelskaufleute oder als Mediziner/in bis zu künstlerischen Berufen). Sie sind nicht immer realistisch in Bezug auf ihre faktische Umsetzbarkeit, dokumentieren den ausgeprägten Wunsch nach Arbeitsmarktintegration und den hohen Stellenwert der Erwerbstätigkeit im Wertesystem. Dem stehen aber zahlreiche Hindernisse in der Realisierung_gegenüber: 
„,... gefällt mir in der Baustelle, weil ich kenne das alles, ... im Iran habe ich so lange gearbeitet, aber leider kein Zertifikat oder so etwas. Aber hier muss ich lernen. Zuerst lernen und dann arbeiten. “ (I25, männlich, *1997, seit 2014 in Ö.)

„Ich würde in einem Büro arbeiten. Ich habe schon in Ferien gearbeitet einen Monat und Ferialjob. Und es war ein Pflichtpraktikum sozusagen und ich habe im Büro gearbeitet und das hat mir sehr gut gefallen. " (I18, weiblich, *1998, seit 2016 in Ö.)

Bezüglich der engen Verflechtung von Ausbildung und beruflicher Integration sei auf den Abschnitt zur Bildungsintegration verwiesen. Ganz analog wie bei Bildungswünschen muss es häufig zu einer Adaption an die Realität kommen. Dieser Prozess wurde vor allem von den bereits länger in Österreich lebenden Befragten beschrieben und vollzieht sich auch bei den rezent Geflüchteten nach ersten enttäuschenden Erfahrungen:

„,... ich bin eine Lehrerin, deshalb mein Wunsch immer war, eine Lehrerin zu bleiben. Aber leider hier ... meine Dokumente, und zwei Abschnitte von meinem Studium wurden anerkannt, dritten Abschnitt ich sollte hier absolvieren. Und damals meine Kinder waren klein, ich konnte das nicht. ... Deshalb ich konnte nicht studieren. Dann habe ich diesen Job gefunden und habe ich gesagt: ,O.K., das passt zu mir. " “ (I23, weiblich, *1949, seit 1988 in Ö.)

Viele Geflüchtete überschätzen die Möglichkeiten, die demokratische Systeme bezüglich der Bildungs- und Arbeitsmarktperspektiven offerieren. Im Zuge ihres Aufenthaltes stellen sie fest, wie schwierig eine Arbeitsaufnahme tatsächlich ist und wie hoch die formalen Anforderungen sind. Etwas, was sie vor dem Hintergrund der facettenreichen informellen Arbeitsmärkte in Afghanistan, in Pakistan oder im Iran in dieser Form noch nicht erlebt haben. Wunschvorstellungen müssen deshalb an die Gegebenheiten angepasst werden. Vielfach helfen hier die NGOs mit entsprechenden Beratungsangeboten.

„Naja, es wird halt realistischer. Also ich glaube schon, dass die Leute die Bildungschancen und auch die Demokratie ein bisschen überschätzen und die Möglichkeiten, die man hat. " (E2)

„Also man hat sehr hohe Erwartungen, weil ... man sieht ja Europa als Vorbild, wenn man nicht jetzt in Europa lebt, sondern in Afghanistan, ... die Menschen denken, sie fliehen, aber es wird dann alles gut gehen und sie können da arbeiten. Und dann umso größer sind die Enttäuschungen, wenn sie dann so viele Hindernisse sehen. "(E8)

„Am Anfang dachte ich, dass ich arbeiten werde. Nachdem ich aber hier war, sah ich die Schwierigkeiten. Es gibt viele Schwierigkeiten und wenig Arbeitsplätze. Zum Beispiel in den anderen Ländern, wenn du Sprache verstehst oder nicht, gibt es eine Arbeit, aber hier zuerst muss man die Sprache lernen, nachher sagen sie, 
dass man warten muss, bis man eine Arbeit gefunden hat. Ich denke, hier gibt es nicht viele Arbeitsplätze. " (I59, weiblich, *1999, seit 2016 in Ö.)

Es kommt des Weiteren zu einer Kollision zwischen der Vorstellung, dass in hochtechnisierten westlichen Gesellschaften einfache manuelle Tätigkeiten völlig überflüssig werden, und der Wirklichkeit. Diese Erwartung steht in einem krassen Gegensatz zu der häufigen Erfahrung Stellensuchender in Bezug auf die offenen Stellen, die vor allem in monotonen, untergeordneten Positionen und manuellen Tätigkeiten, etwa in der Gastronomie, der Reinigungsbranche oder der Produktion, bestehen.

„,Und dann sagen sie diesen Menschen, der einzige Arbeitsplatz, den wir für sie haben, ist als Abwäscher in einem Restaurant. Das heißt, man kommt nach Europa mit einem Traum, da gibt's nur Geschirrspülmaschinen ... Und dann arbeite ich da als Abwäscher in einem Restaurant und das klappt dann gar nicht ... Also, was viele dann mitbringen ist die Hoffnung, dass in Österreich alles gut wird. Dass Österreich alles schafft, dass wenn sie nach Europa kommen, nach Österreich kommen, dass sie nichts machen müssen. "(E8)

Es werden auch realistische Erwartungshaltungen berichtet, wobei der wichtigste Aspekt in der Möglichkeit zum Einkommenserwerb besteht, um Schulden abzuzahlen und monetäre Transferleistungen in die Herkunftsländer durchzuführen.

\subsubsection{Die Stellensuche}

Die Integration in den Arbeitsmarkt wird von allen Expert/inn/en aus ökonomischen und psychologischen Gründen sowie aus der Perspektive gesellschaftlicher Teilhabe als der wichtigste Aspekt der Inklusion klassifiziert. Arbeit und Einkommen wirken sich positiv auf das individuelle „Wellbeing“ aus, stärken das Selbstwertgefühl und vermitteln eine generell positive Lebenseinstellung. Es wird darauf hingewiesen, dass infolge von längeren Phasen der Erwerbslosigkeit das Risiko für psychische Probleme steigt. Dauer und Verlauf der Asylanerkennungsverfahren sind verbunden mit einem permanenten Unsicherheitsgefühl, welches die Betroffenen belastet und der Arbeitsintegration abträglich ist. Die große Zahl jener, die im Gefolge der „refugee crisis“ zuwanderten, sieht sich mit Problemen der Arbeitsintegration konfrontiert, mit welchen vorangegangene, zahlenmäßig viel kleinere Flüchtlingswellen aus Afghanistan in dieser Form nicht konfrontiert waren. Die meisten Befragten zeichnen sich aber durch hohe berufliche Flexibilität aus. Auch jene, die vor ihrer Flucht qualifizierte Positionen bekleideten, sind sehr häufig bereit, zumindest temporär in Tätigkeitsbereichen unterhalb ihres Qualifikationsniveaus aktiv zu werden. Die Wichtigkeit positiver „role models“ erfolgreicher Afghan/inn/en wird von Seiten der Expert/inn/en betont.

„,Also ich glaube die allerwichtigste Integrationsmöglichkeit ist irgendeine Form von Beschäftigung, ob das jetzt wirklich eine Arbeitsstelle ist oder nicht. Aber sozusagen aus psychosozialen Hygienegründen wäre es gut, wenn die Leute irgendwas zu tun haben, wo sie ein Selbstwertgefühl haben. ... Dass sie was zu tun haben, dass sie eine Tagesstruktur haben. Das kann jetzt gemeinnützige Arbeit 
sein, .... Oder man gibt ihnen halt Geld, dass sie das Gefühl haben, dass das, was sie tun, ist auch irgendwie eine Leistung oder bringt was. Da gibt's verschiedene Möglichkeiten. Das AMS hat da viel mehr Möglichkeiten, was zu machen. Die stecken die Leute halt in Kurse, aber für sie sinnvoller ist ab einem gewissen Deutschniveau, dass die Leute dann wirklich Arbeitstrainings bekommen, eine Nachqualifizierung bekommen, weil die gesellschaftliche Integration funktioniert am stärksten über den Arbeitsmarkt. " (E5)

„,... wenn man lang ohne Arbeit ist, das denke ich mir, jeder Mensch, vollkommen egal, ob Mann oder Frau, fühlst du dich wertlos, fühlst du dich einfach ... nicht integriert in die Gesellschaft. Also es ist wirklich ..., dass ein Job einfach das wichtigste ist. Und Sprache, damit sie integriert werden. Also diese zwei Aspekte hören wir ununterbrochen, dass sie einfach nach Arbeit suchen, egal was. " (E4)

„, Und deswegen ich habe schon acht Monate Depression, bleibe zu Hause. ... Und ja, das ist eigentlich sehr wichtig für mich, Arbeit. Wenn ich zu Hause bleibe, das ist für mich sehr, sehr ... " (I20, weiblich, *1964, seit 2013 in Ö.)

Die beruflichen Integrationsverläufe werden von einer Kombination unterschiedlicher „skills“ beeinflusst. Dies können ,social skills“ sein, mit dem Bildungsniveau einhergehende kommunikative Fähigkeiten oder das Wissen um institutionelle Abläufe und Erfahrungen mit institutionellen Strukturen. Genauso kann es sich aber um bereits aus dem Herkunftskontext mitgebrachte Kompetenzen handeln.

„... sie haben sehr hohe Skills gehabt, die Familie. Waren auch eine sehr angesehene Familie in Afghanistan, das ist eindeutig zu merken. Also die, die halt dann schon in Afghanistan aus gebildeteren Schichten kommen, die tun sich da halt schon hundertmal leichter. ... Also ich sehe immer das Hauptproblem ist ... das Bildungsniveau. Das macht den größten Unterschied. Und die tun sich dann auch leichter mit den Ämtern und alles. Die wissen dann: ,Ah, das muss man so machen oder so kommunizieren. 'Und je jünger und je bildungsferner die sind, desto schwieriger wird's. "(E9)

\subsubsection{Hindernisse und Schwierigkeiten der Arbeitsmarktintegration}

Die Hindernisse sind ähnlich wie bei anderen Drittstaatsangehörigen und nicht spezifisch für die afghanische Community: mangelnde Deutschkompetenz, Ausbildungsdefizite, fortgeschrittenes Alter, gesundheitliche Beeinträchtigungen, rechtliche Restriktionen und Xenophobie sind die häufigsten Probleme, welche ein Fußfassen auf dem Arbeitsmarkt behindern. Bei einigen Frauen kommen als zusätzliche Hürden das Tragen des Kopftuchs sowie Kinderbetreuungspflichten hinzu. Einige Befragte kritisieren die aus ihrer Perspektive mangelnde institutionelle Unterstützung, etwa durch das AMS, wobei hierbei nicht generalisiert werden kann.

„Mein großes Problem ist, ich kann nicht sprechen Deutsch ... Und ich viele Male AMS gehabt. Ich bin gegangen und gesagt: ,Bitte geben mir B1-Kurs wieder: Aber er hat gesagt: ,Nein, das ist nicht möglich, du musst arbeiten'. ,OK, ich 
möchte arbeiten, bitte einen Job finden für mich, weil ich habe ein Knieproblem. Ich kann nicht schwierig arbeiten. 'Nur sagt mir ,O.k. ' und bis jetzt ich habe keine B1-Kurs bekommen, Sprachkurs nicht bekommen und auch ich habe keine Arbeit. " (I20, weiblich, *1964, seit 2013 in Ö.)

„,... und für mich nicht Deutschkurs bekommen mehr. Das nur ein-oder zweimal bekommen. Und jetzt brauche ich einen B2-Kurs, weil ich weiß, wenn ich zum AMS gehe oder zum Sozial oder zu anderen Leuten, sie sagen mir: ,Nein, wir können dir nicht helfen. ' ... weil das ist von ÖIF, ich habe einen Berater. Er hat einmal für mich eine Aussage gesagt, wenn ich das alles Papier gemacht und zu ihm gegangen. Er sagt: ,Nein, wir brauchen nicht das. Du musst das machen, du musst das machen. "“ (I19, weiblich, *1973, seit 2011 in Ö.)

Analog zu Erfahrungen bei der Wohnungssuche berichten Asylberechtigte zudem, dass ihnen bei der Suche nach einer Beschäftigung Vorurteile entgegengebracht wurden. Das Image der Community und die Auswirkungen der medialen Berichterstattung spüren viele Befragte und da innerhalb der Projektlaufzeit auch die Wahlen zum Nationalrat stattfanden, ging auch der politisch-mediale Diskurs um Fluchtmigration nicht spurlos vorüber. Die Herausforderungen, mit denen sie bezüglich der Arbeits- und Wohnungsmarktintegration zu kämpfen haben, werden dadurch verschärft.

„Es ist, durch die ganzen negativen Sachen, was in den Zeitungen gestanden sind. Also Afghanen und Tschetschenen haben sehr schlechte Karten bei dieser Sache. Auch bei der Arbeit, ist genau dasselbe. " (E7)

„,Und am Arbeitsmarkt ist, glaube ich, weniger Diskriminierung wie am Wohnungsmarkt. Weil am Arbeitsmarkt, weiß ich nicht, habe ich so das Gefühl, dass da so mittelfristig die Leute dann schon Jobs finden ... “ (E2)

ich hoffe, dass wir Afghan/inn/en nach der Wahl leichter Wohnungen finden und leichter einen Job finden können. Ich hoffe, dass sie nicht so stark in den Medien [vertreten] sind. “ (E12)

„,... Job finden die Afghan/inn/en leider (nicht), weil sie ein anderes Bild haben innerhalb der Gesellschaft. Durch ORF-Sendungen „Am Schauplatz“, durch andere Artikel, die veröffentlicht wurden, durch andere Artikel, wo Afghan/inn/ en vorgestellt werden. Obwohl nur ein paar Personen so sind, nicht alle Afghan/ inn/en, alle sind in diesem Namen und die bekommen keinen Job, keine Wohnung, haben keine Perspektive, keine Möglichkeiten, sie sind einfach auf der Straße." (E12)

„Ich habe einen jungen Mann kennengelernt, der hat gesagt: ,Frau XX, ich möchte drei Sachen machen: Ich möchte meinen Namen ändern. Ich möchte auf meinem Lebenslauf nicht mehr stehen haben, dass ich aus Afghanistan komme und ... warum fragt mich jeder nach meiner Religion?' Er hat gesagt: ,Wissen Sie, es ist so schwer, XY zu heißen, aus Afghanistan zu kommen, Religionsgruppe Islam 
zu haben. Wissen Sie, das ist kaum möglich. Nirgends finden Sie einen Arbeitgeber, der eine Person mit diesen drei Möglichkeiten einstellt. “ " (E8)

Weitreichende Auswirkungen auf die Arbeitsintegration hat die „visibility“ in Form der Verhüllung von muslimischen Frauen. Die (wenigen) Befragten, die auf Verhüllung bestehen, sind sich des Handicaps bewusst, wollen die Konsequenzen aber auf sich nehmen.

„,... es ist nur beim Arbeiten suchen, es ist ein Problem. Mit meinem Kopftuch kann man nicht arbeiten, in vielen verschiedenen Beispielen, Bereichen. Aber ich kämpfe. " (I24, weiblich, *1996, seit 2015 in Ö.)

\subsubsection{Arbeitsmarkteinstieg: institutionelle Unterstützung}

Die Herausforderungen bei der Arbeitssuche sind vielfältig. Die Wichtigkeit der Unterstützung durch diverse NGOs und engagierte Freiwillige bei der Nutzung von Bildungsmöglichkeiten und der beruflichen Integration wird von Expert/inn/ en durchwegs betont. Wichtig sind die institutionellen Beratungsangebote, denn von zahlreichen Institutionen werden Programme angeboten, welche die Anrechnung von Bildungsabschlüssen sowie die berufliche Integration erleichtern.

„Einzig in Oberösterreich, die da lange Tradition haben die Arbeitsämter bei der Integration von Flüchtlingen und prinzipiell Offenheit. Da wiederholt sich das, was schon bei den Bosniern und so weiter sich abgespielt hat. Dass dort schon, durch die spezielle Struktur auch, dass das halt sehr wohlhabende Gebiete sind mit vielen Arbeitsplätzen. Dass da, glaub 'ich, schon mehr bleiben. ..., dass die Sozialpartner in Gemeinden gehen und dort sozusagen die Beschäftigung von Flüchtlingen anpreisen. Da wird viel proaktiv gemacht für die Integration. Und das ist außer in Oberösterreich und in Wien nirgends so massiv, diese Maßnahmen. " (E2)

„,... wenn man dann einen Asylbescheid hat, dann ist es natürlich einfacher dieses Programm durch AMS. ... Aber WAFF hat ein tolles Programm in Verbindung mit AMS, dass sie einfach diesen Check machen: Hatte diese Person in ihrer Heimat studiert? Kann man diese Dokumente anrechnen lassen hier? Die schauen das genau an, also die nehmen sich Zeit, begleiten diese Person. “ (E4)

„,... der dritte Schwerpunkt ist - da sind wir jetzt hauptsächlich bei den Afghanen - das Nützen der wenigen Möglichkeiten, die wir im Bereich Beschäftigung haben, .... Die beste Möglichkeit ... ist derzeit die Lehrstelle für Asylwerber in Bereichen, in denen Mangelberufe deklariert sind, und da schaut's recht gut aus in Oberösterreich. Da samma mittlerweile bei knapp 300 Lehrstellen und das Schöne daran ist, was mich sehr fasziniert, dass das zu einem ganz großen, überwiegenden Anteil Afghanen sind. Und davon sogar die ersten drei Mädchen aus Afghanistan, dieund das woll' ma halt-möglichst, möglichst pushen, möglichst stärken, weil das auch ein sehr tolles Thema ist, anzukommen und vor allem Lebensperspektive zu kriegen. "(E10) 
„,... sie gehen zu ihren AMS-Terminen und erwarten, dass AMS ihnen dann eine Stelle in die Hand gibt ... Nach der 50. Bewerbung bekommen sie noch immer keine Antwort. Das wird dann immer schwieriger und schwieriger werden. Die sind dann mit sich beschäftigt. " (E8)

„Integrationsfonds hat uns sehr geholfen und wir haben einmal in der Woche eine Sitzung und hat uns in den Bereichen Arbeitssuche, Spracherwerb beraten. " (I60, weiblich, *1960, seit 2006 in Ö.)

„I: Und wie hast du diesen Job bekommen? B: Auch durch Volkshilfe. ... Durch Kontakte, weil ich war ja dort immer aktiv. “ (I4, männlich, *1985, seit 2001 in Ö.)

„,... meine Sozialarbeiterin hat gesagt: ,Frau X. willst du einen Job suchen? Ich hab 'gesagt: ,Ja'. Sie hat mir Inserat gegeben ... Ich hab ' gesagt: ,Ja, das will ich einmal probieren. 'Sie hat mir gesagt: , Gehst du ins Büro und dann Telefon selber anrufen und selber vorstellen. 'Dann haben ich mich so gesetzt und mit vollem Mut gesprochen mit Chefin von dem X. [Lebensmittelkette] in 14. Bezirk und ich hab ' mich vorgestellt und dann hab'ich gesagt: ,Ich hab 'Interesse am Job, will ich probieren. 'Bin ich gegangen und sie hat mir Formulare gegeben. Habe ich sofort gegeben unterschreiben und sie hat freundlich gesagt: ,Kommst du Montag, Arbeit. 'Und dann nach zwei Wochen ich hab' auf meine Füße stehen in die Arbeit hab'ich alles gelernt, ... ich freue mich auf diesen Job. Nur wegen der Probleme wegen Schmerzen, vielleicht, aber ich will nicht gerne verlassen meinen Job. ... nächstes Jahr ist 15 Jahre, bin ich bei X. [Lebensmittelkette]. “ (I39, weiblich, *1967, seit 1999 in Ö.)

\subsubsection{Arbeitsmarkteinstieg: Unterstützung durch soziale Netzwerke}

Analog zu den beiden anderen strukturellen Integrationsbereichen der Ausbildung sowie des Wohnungsmarktes spielt auch bei der Stellenfindung die Unterstützung durch soziale Netzwerke eine wichtige Rolle. Einen Nachteil stellt der vergleichsweise geringe Organisationsgrad der afghanischen Community in Österreich dar. Dies unterscheidet die österreichische Situation von jener in UK oder Deutschland, wo das Vorhandensein zahlreicher Unternehmen der ethnischen Ökonomie es den Geflüchteten erleichtert, beruflich Fuß zu fassen. Dazu kommt in anglophonen Ländern, dass eine zusätzliche sprachliche Hürde wegfällt. Aufgrund des Mangels an einer ausdifferenzierten afghanischen Migrant Economy in Österreich sind für das Gros der Befragten Kontakte zu Österreicher/inne/n bei der Suche nach Arbeitsplätzen umso unentbehrlicher. Social Networking mit Österreicher/inne/n ermöglicht Hilfestellungen jeglicher Art. Auch Kombinationen mehrerer Suchstrategien sind relevant, z.B. Hilfestellungen durch Freund/e/innen, Inanspruchnahme von Beratung seitens der NGOs, das Checken von Online- und Printmedien-Stellenofferten.

„Es sei denn sie sind sehr intensiv betreut und es kümmern sich irgendwelche Mentor/inn/en oder Pat/inn/en ... oder sie kommen schon aus Familien mit einem 
massiven Bildungshintergrund. ... Ich kenne jetzt schon einzelne Fälle, wo sie Lehrplätze irgendwo gekriegt haben ... “(E2)

„Die brauchen Unterstützung von Anfang an, so wie in meinem Leben. ... Wenn sie die Unterstützung und die Hilfe nicht gehabt hätten, hätten sie die Chancen nicht gehabt, dass sie ihr Leben hier aufbauen, etwas Positives für Österreich zu tun und nicht arbeitslos werden. Es gibt schon Leute, die diese Möglichkeit nicht haben und die sind leider zwei Monate in einer Arbeit, drei Monate arbeitslos und sechs Monate einen Kurs besuchen, sechs Monate arbeiten, sechs Monate arbeitslos. ... die leben einfach so. " (E12)

„Es ist eine große Hilfe für einen, wenn man viele Kontakte hat. Und wenn man österreichische Freunde hat, ist dies auch sicherlich eine große Hilfe in Bezug auf Arbeit und vor allem beim Lernen. " (I65, männlich, seit 2015 in Ö.)

„Die Afghanen haben ja nicht so viel geholfen. Aber was die Lehre anbelangt, hat mich nur eine Österreicherin beraten und gesagt, dass es auch andere Lehrstellen gibt, weil ich unterschiedliche Praktika gemacht habe und keine gemocht habe ... das Praktikum als Orthopäde habe ich am Ende der Woche gemacht und habe mit den Leuten dort gesprochen und es gefiel mir. Da haben sie mich akzeptiert und dann habe ich begonnen hier zu arbeiten. “(I51, männlich, *1993, seit 2010 in Ö.)

\subsubsection{6 (Aus-)Bildungsdefizite}

Unter Expert/inn/en besteht Einigkeit, dass sich besonders das geringe Bildungsniveau vieler Asylberechtigter in der afghanischen Community negativ bemerkbar macht. Höhere Schul- und Berufsausbildungen eröffnen ein breiteres Spektrum an Möglichkeiten, steigern die berufliche Flexibilität und erlauben die Abwägung beruflicher Alternativen. Andererseits ist die Nachfrage im Bereich einfacher manueller Tätigkeiten in vielen Branchen vorhanden und entspricht somit eher dem, was viele Geflüchtete in den Herkunftsländern (v.a. im Iran) gemacht haben. Gebildete erleben vor allem Dequalifikation. Die Bandbreite an Problematiken wird auch in den Analysen zur Bildungsintegration angesprochen.

„,... die Arbeitsmarktintegration, das ist wirklich mit den Afghanen glaub 'ich die größte Herausforderung, weil viele halt einen sehr niedrigen Bildungsabschluss haben, manche gar keinen. Viele der Afghanen, die nach Österreich gekommen sind, kommen eigentlich aus dem Iran und nicht direkt aus Afghanistan und die waren ja dort auch ausgegrenzt oder zumindest hatten keinen einfachen Zugang zum staatlichen Bildungssystem. Dadurch kommen die halt relativ oft zu uns und sind ... auf Dari oder Farsi halt wacklig oder gar nicht alphabetisiert. Und je höher der Bildungsgrad ist, umso leichter finden sich die Leute natürlich zurecht. Die brauchen dann weniger Hilfe. Wenn jetzt jemand kommt mit einem Universitätsabschluss, den schickt's halt irgendwohin, der macht das schon. Also um den braucht man sich da irgendwie nicht so viele Sorgen machen. " (E5) 
„,Da ist zum Beispiel ein Projekt, wo sich Graz überlegt hat: ,Na ich habe ja viele Menschen, die zwar ... Analphabeten sind, aber wo kann ich sie einsetzen?' Und dann haben sie sie in der Forstwirtschaft eingesetzt und die sind auch glücklich und da waren glaub'ich zwei Menschen aus Afghanistan, einer oder zwei Menschen aus Syrien, die dann in dem Bereich arbeiten. Und die sind handwerklich begabt, brauchen nicht sehr viel die Sprache und sie arbeiten, sind glücklich. .... " (E8)

„Die Population von afghanischen Asylwerbern ist natürlich sehr breit gestreut. Das heißt, wir haben zum Teil sehr hochgebildete Personen, die in Top-Positionen in der Regierung oder in NGOs in Afghanistan waren und fliehen mussten. Und wir haben Analphabeten, die vielleicht Berufserfahrung haben, aber in Afghanistan gibt es auch nicht dieses System von Berufsschule und Arbeit in einer Firma und entsprechende Zertifikate. ... Und dazu kommt auch, dass das Segment des Niedrigstlohnbereiches ja eigentlich schon besetzt ist, auch von vielen EU-Bürgern aus Südosteuropa, so dass diese Jobs dann oft wegfallen. Und umgekehrt die Hochqualifizierten eine Dequalifizierung erleben, weil die Nostrifikation teuer und langwierig ist und man dann dennoch oft nicht den wirklichen, der Ausbildung entsprechenden Job finden kann. ... Das Arbeitsmarktservice geht davon aus, dass es mindestens fünf Jahre dauert, bis ein Teil der Afghanen in den Arbeitsmarkt integriert ist, wobei anscheinend Studien zeigen, dass die Afghanen weniger wie soll ich sagen? -selektiv sind, was Jobs angeht. Auch schlecht bezahlte Jobs übernehmen, was bei Syrern weniger der Fall ist. Also die haben eine höhere Arbeitslosenquote unter den anerkannten Asylwerbern als die Afghanen. " (E1)

\subsubsection{Der „Refugee Gap“}

Die komplexen Konstellationen, welche für den „refugee gap“ verantwortlich sind, werden in zahlreichen Interviews angesprochen. Eine vorgeschaltete legistische Hürde auf dem Weg zur Integration in den Arbeitsmarkt bilden die gesetzlichen Rahmenbedingungen der Anerkennung von Qualifikationen, deren Auswirkungen jedoch zusätzlich durch sprachliche Defizite und kulturelle Zuschreibungen verschärft werden. Die Akkumulation diverser Herausforderungen in strukturellen und sozialen Integrationsbereichen wird im Folgenden beschrieben:

„Als Erwachsener, sie haben dann mit so vielen Schwierigkeiten zur selben, zur gleichen Zeit zu kämpfen, dass da so wenig Platz bleibt, um die Sprache zu lernen in der Zeit. Das muss ja eigentlich alles parallel funktionieren, weil wir so schnell unterwegs sind. ... Das ist mal eine große Herausforderung mit den Terminen und so, die sie am Anfang erleben. ... Dann kommt der Druck, sie müssen Deutsch lernen, arbeiten. Und sie können nicht, wenn sie ein Zimmer haben und fünf Kinder haben, dass sie im Deutschkurs lernen. Dann können sie nicht nach Hause gehen, in einer Ecke sitzen und die Sprache weiterlernen. " (E8)

„,... Und ich fange hier mit Null. Das ist aber ganz schwer. Sprache lernen, Kultur lernen, das ist ... wenn ich in meinem Heimatland leben könnte, .... Was ich habe 
dort gelernt ..., das funktioniert hier einfach nicht. ... ich habe dort als Trainer gearbeitet, ich muss hier als Bausteller [Anm.: auf der Baustelle] arbeiten. Wenn ich Arbeit nicht finde, ich muss dann arbeiten, ja? Oder Putzmann oder ... ich weiß nicht, was ich finde, dann werde ich arbeiten. " (I8, männlich, *1989, seit 2012 in Ö.)

Auch bei hoch motivierten Geflüchteten ergeben sich Hindernisse in der Bildungslaufbahn, welche den Übertritt in den Arbeitsmarkt erschweren. Deutschkenntnisse sind eine wesentliche Voraussetzung für den Antritt einer Lehre, die dann wiederum Jahre dauert, bis eine dauerhafte Beschäftigung möglich wird. Bildungsmotivierte Befragte empfinden es daher als ungerecht, wenn in der Medienberichterstattung Geflüchteten pauschal vorgeworfen wird, sie würden arbeitsunwillig sein.

„Ich lese manchmal in den Zeitungen, dass die österreichische Regierung sich darüber beschwert, dass die Flüchtlinge nicht arbeiten wollen und dass sie arbeitslos sind. Ich möchte der österreichischen Regierung sagen, dass sie uns diese Möglichkeit erst nicht ermöglicht, dass eine Person eine Lehre machen kann. Und wie soll die Person denn arbeiten, wenn diese gar nicht arbeiten darf und der österreichischen Gesellschaft was zurückgeben? " (I54, männlich, *1996, seit 2015 in Ö.)

Hürden in der (Fortsetzung der) Bildungslaufbahn und der Verlauf der Arbeitsintegration sind eng verzahnt. Das gesamte Spektrum an Herausforderungen umfasst neben dem Erlernen der Sprache das Vertrautwerden mit einer unbekannten Kultur und deren Normenkodex, den Aufbau neuer sozialer Netzwerke sowie die Bewältigung der Schwierigkeiten eines Universitätsstudiums. Nach dessen Abschluss tritt oftmals eine krasse Dequalifikation ein, indem „klassische“ Beschäftigungen vieler Geflüchteter in der Gastronomie vor allem aus finanziellen Gründen gewählt werden müssen. Die Flucht bewirkt ohnehin regelmäßig eine Entwertung mitgebrachter Qualifikationen, dies gilt vor allem auch für Juristen und Mediziner, weniger für Techniker und Naturwisssenschafter.

„Zum Beispiel, wenn man Jus studiert hat in Afghanistan, islamisches Recht und so weiter, ist ganz ein Unterschied zu dem, und man konnte damit auch nicht viel arbeiten. Das ist eine Hürde gewesen. “ (E8)

Wiederkehrende Brüche in der Berufslaufbahn sind daher das Merkmal vieler Berufsbiographien von Geflüchteten:

„Es gibt schon Leute, die diese Möglichkeit nicht haben und die sind leider zwei Monate in einer Arbeit, drei Monate arbeitslos und sechs Monate einen Kurs besuchen, sechs Monate arbeiten, sechs Monate arbeitslos. Die leben einfach so. “ (E12)

Der Druck, der ausgeübt wird, um die Geflüchteten rasch in Beschäftigung zu bringen, führt ebenfalls dazu, dass Bildungswege abgebrochen werden. Seitens des 
AMS wird rasche Arbeitsmarktintegration gefördert, aber nicht der Abschluss einer Universitätsausbildung. Dies liegt außerhalb der Zuständigkeit des AMS, was für viele Geflüchtete schwer nachvollziehbar ist.

„, Und mein Problem, dass ich gedanklich immer daran hing unbedingt also eher intellektuell tätig zu sein und ... meine Dissertation zu Ende bringen. ... Aber das wurde von Behörden in Österreich nicht akzeptiert. Die haben eigentlich von Anfang an von mir verlangt, dass ich unbedingt arbeiten muss. Allerdings in dem Bereich, wo ich wollte. Egal was, ich musste arbeiten. Und das ist auch der Grund, warum ich nicht weiter studieren konnte. " (I37, männlich, *1952, seit 1988 in Ö.)

„Ist besser AMS wegen arbeiten, wegen Job, wegen Kurs ..., aber ... dann ich will nicht Kurs machen ... mach 'ich auch weiter in Universität, studieren, lernen und so. Aber AMS ... kann nicht helfen in diesem Bereich. " (I41, weiblich, *1998, seit 2015 in Ö.)

Als Gegenstrategie gegen den „refugee gap“ wird es von Expert/inn/en positiv bewertet, Geflüchtete höher zu qualifizieren, als sie rasch und dequalifizierend zu beschäftigen. Dies ist längerfristig zum Vorteil der betroffenen Person und der Volkswirtschaft. Dazu kommt, dass in Wien die Konkurrenz um Arbeitsplätze im niedrigqualifizierten Sektor hart ist.

„, Und der Ansatz ist eher der, wenn man sagt, die bringen Schulbildung mit oder bringen Berufserfahrung mit, dass man sagt: ,Ok - beim AMS - ok, du gehst jetzt nicht Hilfsarbeiterjob ,hackeln', sondern wir versuchen dich auf dein maximales Niveau zu qualifizieren. '... Das dauert zwar länger, aber macht mehr Sinn für die Person und macht auch mehr Sinn volkswirtschaftlich. Also der Druck am Arbeitsmarkt ist in Wien auch grad im niedrigqualifizierten Bereich viel höher als bei Facharbeitern. “(E5)

Mitunter tun sich weniger Qualifizierte leichter bei ihrer beruflichen Verankerung in Österreich. Sie sind es gewohnt, schwere Arbeiten zu übernehmen, für die hierzulande ohnehin nur wenige Bewerber/innen zu finden sind.

„Im Iran haben sie auch zwar keinen Status gehabt, aber sie waren überall Hilfsarbeiter. Sie haben zwar wenig schulische Ausbildung, aber sie können sehr gut arbeiten, weil sie das gewöhnt sind. ... mit der Gruppe, die dann nach Europa gekommen sind, alle wollen arbeiten. Sie schaffen es auch, nur sie tun sich sehr schwer, die Sprache dann zu lernen. "(E8)

\subsubsection{Erfolgreiche Berufsbiographien}

Den Gegenentwurf zum Refugee Gap bilden Beispiele erfolgreiche Berufsverläufe bereits im Rahmen früherer Zuwanderungswellen nach Österreich migrierter Afghan/ inn/en. Aus ihnen kann geschlossen werden, dass auch die rezente Zuwanderung vielfach in stabile Berufsverläufe mündet, wenn persönliche Voraussetzungen sowie positive 
Rahmenbedingungen dies ermöglichen. Wesentliche Faktoren hierfür sind Bildungsressourcen, Aufstiegsmotivation, Durchhaltevermögen etc. Stabile Beschäftigung und Einkommen schaffen die Grundlage für einen friktionsfreien Integrationsverlauf.

„,Dann sind wir kontaktiert worden: , Wollt ihr diese Ausbildung machen-Maschinenschlosser?' Dann haben viele zugesagt und zirka 200 Leute an dieser Maschinenschlosserausbildung haben teilgenommen in Mödling und ... alle haben diese Ausbildung fertiggemacht und viele arbeiten noch immer... Ich habe selber auch das gemacht, hab 'dann auch zehn Jahre als Maschinenschlosser gearbeitet ... Ich habe meinen Taxischein gehabt und bin Taxi gefahren am Wochenende und dann bin ich zum Taxifahrer geworden ... Da habe ich mich selbständig gemacht, eine Konzessionsprüfung gemacht, habe dann auch mehrere Taxis gekauft ... “ (E7)

„Ich habe zuerst beim XX [Lebensmittelkette] gearbeitet. Und dann habe ich bei YY [Lebensmittelkette] angefangen. Und ich arbeite als Stellvertreter von Feinkost. Es ist gut. Ich verdiene besser. Aber trotzdem ist es nicht mein Traumjob. Ich mache das nur für die Familie. Ja, aber es ist gut, wenn man arbeitet, was man will in dem Bereich. Dann macht man seine Arbeit gerne. Aber jetzt ... " (I1, männlich, *1989, seit 2013 in Ö.)

\subsubsection{Arbeitsbezogene Werthaltungen}

Arbeits- und Erwerbsmotivation

Die Arbeitsmotivation der Geflüchteten ist durchwegs sehr hoch. Die weit verbreitete Praxis der Kinderarbeit in Afghanistan sowie in Iran und Pakistan prägt in der afghanischen Community durchgehend ein traditionelles Arbeitsethos, welches ein Leben ohne Arbeit als nicht vorstellbar erscheinen lässt. In keinem Fall wurde die Aufnahme einer Beschäftigung negativ bewertet. Die ausgeprägte Bildungs- und Arbeitsmarktorientierung wird auch von HosNER et al. (2017, p. 88) bestätigt, wobei die Erwerbsmotivation durch bereits erwähnte Barrieren gebremst wird. Bei Frauen zusätzlich durch Kinderbetreuungsverpflichtungen oder tradierte Rollenmuster.

„Weil bei den Afghanen ist das Arbeiten, Beschäftigung einfach etwas, das wichtigste Thema. Es gibt keinen Kurs, wo wir reingehen, unsere Referenten hingehen, wo die nicht sagen: ,Wie komm ich zu einem Job? ... Ich will kein Geld, ich will nur beschäftigt sein. 'Weil die einfach gewohnt sind als Kind zu arbeiten und das Leben ohne Arbeit, ohne Beschäftigung, ohne irgend aktiv zu sein ist nicht lebenswert. "(E4)

„Weil es ist ja wichtig, dass man arbeitet ... genug verdient.“ (I15, weiblich, *1993, seit 2001 in Ö.)

„,Meinen Mann hat das auch überzeugt und hat er sich dazu entschlossen nach Österreich zu kommen. Dann bin ich nachgekommen. Und es hat mir sehr gut gefallen hier. Das Einzige, was mir nicht so gut gefallen hat war, dass ich hier nicht arbeiten kann. “... „Ich mag kein mindestsicherungsabhängiges Leben, ich 
will keine soziale Hilfe in Anspruch nehmen. Ich fühle mich sehr wohl, da in Österreich Sicherheit herrscht, ... jeder muss in Österreich arbeiten und Steuern zahlen und nicht von Mindestsicherung leben. " (I57, weiblich, *1966, seit 2015 in Ö.)

Die Geflüchteten wollen beruflich aktiv sein, werden aber in der Realität mit beschränkten Chancen konfrontiert. Hervorzuheben ist, dass kaum ein Absinken der Erwerbsmotivation im Verlauf des Aufenthalts in Österreich oder bedingt durch Frustrationen im Rahmen der Arbeitsfindung verbalisiert wurde. Die Erwartungen werden lediglich an die realen Möglichkeiten angepasst und sukzessive reduziert.

Expert/inn/en sehen Veränderungen gegenüber früheren Zuwanderungswellen, denn heute wird mehr institutionalisierter Druck zur Aufnahme einer Beschäftigung ausgeübt. Die Sozialhilfe war in früheren Jahrzehnten für einen quantitativ nicht bestimmbaren Teil der Geflüchteten als Lebensgrundlage ausreichend.

„Und nachdem sie nicht alle Asyl bekommen, bekommen sie subsidiären Schutz oder Visum oder irgendwas, da sind sie auch gezwungen zu arbeiten. ... Aber früher waren sie nicht so motiviert, weiter zu arbeiten, weil Sozialhilfe ihre Bedürfnisse gedeckt hat und 1150 Euro als Gehalt für 40 Stunden war uninteressant. ... Jetzt wollen sie alle arbeiten. “ (E3)

\section{Erwerbstätigkeit und Gender Role Models}

Bei traditionell denkenden Afghan/inn/en mit einem konservativen Rollenverständnis kann eine Kollision von Wertvorstellungen eintreten, wenn die Erwerbstätigkeit von Frauen das tradierte Rollenverhalten in Frage stellt. Die finanziellen Vorteile werden zwar wahrgenommen, aber es benötigt Zeit, bis dies Selbstverständlichkeit gewinnt. Gegenwärtig dominieren bei einem Teil der Befragten traditionelle Rollenkonzepte, wobei diese von urbaner oder ländlicher Herkunft, vom Bildungsniveau und spezifischen Gepflogenheiten in der Herkunftsfamilie abhängen. Die Veränderung der Rollenbilder in Bezug auf die Erwerbstätigkeit ist stark von der Dauer des Aufenthaltes abhängig. Hohe Erwartungen bezüglich der beruflichen Möglichkeiten werden auf ein realistisches Niveau reduziert (vgl. dazu auch das Subkapitel zu den Erwartungen). Dies gilt für beide Geschlechter, wenn erlebt wird, wie schwierig die Arbeitsmarktsituation für Männer und Frauen gleichermaßen ist:

„Nur die Afghanen haben doch eher die Tendenz, dass sie sehr konservativ sind und ihre Frauen dann nicht arbeiten lassen. Und wie weit Heimarbeit möglich ist ... Also viele Afghan/inn/en haben ja früher auch daneben verdienen müssen. Die haben halt zu Hause genäht, zum Beispiel. Aber dieses Segment ist eigentlich besetzt, weil die meisten Schneider in Wien, zumindest die so Reparaturschneider sind, sind Türken. " (E1)

„,Und ich glaube die Menschen tun sich genauso schwer, wenn sie nach Österreich kommen. ... mittlerweile kommen zu mir ... junge Frauen, die gearbeitet haben, studiert haben, ... Für die ist überhaupt kein Thema, dass sie arbeiten. ... dass 
es für denen schon so klar, dass die Arbeitsmarktsituation so ist, dass sie einfach keine Arbeit finden. ... und das ist dann schwer für mich dann zu sagen, wie sie jetzt sich dann in diesem Rollenbild ... “ (E8)

„,... ich glaube, jeder möchte, dass seine Frau zu Hause ist und er viel Geld verdient und bringt das Ganze nach Hause und so und die Frau passt auf die Kinder auf. ... Und die werden dann auch irgendwann einmal merken, wenn sie da leben, wenn er selber alleine arbeitet, der wird mehr z'sammbringen, wenn die Frau dann mithilft, dann wird es besser werden. Also sie werden da sicherlich irgendwann einmal draufkommen. ... Aber zurzeit ist irgendwie das Traditionelle - Frau zuhause und so. Aber das wird sich dann anders entwickeln. Ich kenne schon sehr viele Frauen von Flüchtlingen, die arbeiten. “(E7)

„Ich kenne viele afghanische Frauen, die hier mehr tätig sind, aktiver sind als die Ehemänner. Die Ehemänner zu Hause bleiben, weil die keine Möglichkeit, Arbeitsmöglichkeit bekommen haben und die Frauen arbeiten. ... Wenn eine Stadt das bietet, nutzen sie es auch. “(E4)

„, [Atmet tief durch] Ich wollte gerne hier arbeiten. Einfach Arbeit oder Lehrstelle von Friseur. Das ist mein Wunsch. I: Und finden Sie alle Frauen sollten arbeiten? B: Natürlich, ja es ist besser [geflüstert]. “ (I42, weiblich, *1992, seit 2014 in Ö.)

Die Erwerbstätigkeit der Frauen stellt für diese durchwegs ein wesentliches Empowerment dar. Dies gilt auch dann, wenn die Tätigkeit in Branchen mit wenig Berufsprestige und auf unteren Ebenen der Beschäftigungshierarchie erfolgt. Das eigene Einkommen macht sie unabhängiger, in manchen Fällen werden sie auch zur Alleinverdienerin im Haushalt. Mit der Stärkung ihrer ökonomischen Position geht ein Statusgewinn einher. Dies kann zu einem Problem in den Geschlechterrelationen, welches sich für die Männer in Form eines Machtverlustes manifestiert, führen. Die Frauen sind also trotz Doppelbelastung die Profiteurinnen der Arbeitsmarktintegration.

„, Sobald sie in Europa sind, dann merken sie: ,Aha, ich bin auch ein Mensch, mit jedem Recht, das ein anderer hat, mit jeder Verpflichtung. ' Verpflichtung haben die Frauen immer in Afghanistan, aber die Rechte haben sie nicht. Und das ist schon für die Frauen von großem Vorteil. ... Und wenn sie hierherkommen, haben sie keine Scheu, im McDonalds als Putzfrau zu arbeiten. Das ist ein Luxus für sie, das ist ganz normal. Sie verdienen ... zum ersten Mal in ihrem Leben ein selbständiges Geld auf ihrem eigenen Konto. Die Männer machen das nicht, haben es schwer. Die Männer, die dort eine Arbeit hatten, die ältere Generation, sagen wir mal über 30, haben es schwer, weil sie dort immer der Befehlshaber waren sozusagen, Befehlsgeber. Und jetzt haben sie es schwer, jetzt befehlen sie nicht mehr. Auf der Straße als Straßenkehrer zu arbeiten ... Für die Frau ist es kein Problem, weil sie immer Befehlsempfänger war. Und daher ist es eine große Errungenschaft für die Frau. "(E3) 
Die Plädoyers für die weibliche Berufstätigkeit sind zahlreich und nicht ausschließlich ökonomisch legitimiert. Einige Respondent/inn/en beziehen sich auf die in Österreich geltenden Normen:

„,Wir sind nicht in Afghanistan, wir sind in Österreich, und wir müssen so machen, wie das ist hier. ..., wie das ist in einem Land. Z.B. hier muss meine Frau arbeiten, sie kann arbeiten. Ich bin nicht gegen das. Sie hat Deutsch gelernt und weiter sie kann arbeiten. Ich arbeite auch, und zu Hause ich helfe immer meiner Frau. " (I26, männlich, *1974, seit 2013 in Ö.)

Viele junge Männer haben aus ökonomischer Perspektive nichts gegen eine berufliche Tätigkeit ihrer Frau einzuwenden. Bei älteren Männern spielen nicht nur Traditionen, sondern auch realistische Einschätzungen eine Rolle, da die Berufschancen schlecht ausgebildeter Frauen auf dem österreichischen Arbeitsmarkt gering sind. Inwieweit sich dahinter auch ein nicht offen artikuliertes, traditionelles Rollendenken verbirgt, muss unbeantwortet bleiben.

„,Manche Männer umgekehrt, wenn sie kommen und sagen: ,... Meine Frau soll auch bitte arbeiten und dass sie dann ihr auch ein bisschen ... sagen wie das System in Österreich funktioniert und so. '... Also ich hab'viele, viele junge Männer erlebt, auch ältere ... bei den älteren ist schwieriger, weil sie wissen, sie kennen den Arbeitsmarkt ja und sie wissen, wenn sie sich so schwer tun, dass die Frau, die keine Schule besucht hat, noch schwieriger haben. Aber bei den jungen Männern ist es ganz normal ... " (E8)

Im Kontext der Wertehaltungen, welche für die Arbeitsintegration relevant sind, sind jene Rollenschemata wichtig, die Ausbildungs- und Berufswahl determinieren. So ist es für gebildete Frauen zwar selbstverständlich, eine berufliche Tätigkeit anzustreben, allerdings besteht, wie bei Inländerinnen, eine Distanz zu technischen Berufen. Es werden eher klassische weibliche Berufsfelder im sozialen und erzieherischen Bereich präferiert. Nur vereinzelt entscheiden sie sich auch für als „Männerberufe“ konnotierte Beschäftigungen.

„Junge Frauen, die gearbeitet haben, studiert haben, gearbeitet ... Für die ist überhaupt kein Thema, dass sie arbeiten. Natürlich, man kann ja sagen in vielen Berufen ist es ein Unterschied. Wenn ich dann sage, Frauen und Technik. Es sind wenige Frauen, die sagen, Ok'. Aber diese Entwicklungen erleben wir auch in Österreich. Viele Frauen kommen, die sagen: ,Ich will als Kindergartenlehrerin oder Kindergartenpädagogin oder Volksschullehrerin arbeiten, weil ich ja Lehrerin gewesen bin. 'Also so typische Frauenberufe. " (E8)

Eine wichtige Rolle spielen Bildungsangebote und die Möglichkeiten zu deren Nutzung durch Frauen. Sofern infrastrukturelle (v.a. Kinderbetreuungseinrichtungen, denn nur über deren Vorhandensein ist weibliche Erwerbstätigkeit möglich) und bildungsmäßige (Deutschkurse) Rahmenbedingungen geboten werden, werden diese von den Frauen genutzt. Das Spektrum der Angebote hat sich im Vergleich zu vorange- 
gangenen Zuwanderungsphasen erweitert und die Nutzung ist auch den Frauen heute in einem höheren Maß möglich:

„,Und dann hängt's natürlich auch davon ab, dass auch die Schwierigkeit ist die Kinderbetreuung. Also sagen wir in Afghanistan, wenn da jemand arbeitet, auch außer Haus zum Beispiel in gebildeteren Familien, dann gibt es noch die Großfamilie. ... Da gibt es immer eine Infrastruktur und die gibt es da nicht. " (E1)

„Jetzt Gott sei Dank werden Deutschkurse mehr auch für Frauen angeboten, dass die Frauen auch Deutsch lernen. Wenn ich vergleiche, vor 30 Jahren haben Männer sofort Deutschkurse besucht und in die Arbeit gekommen. Es gibt Frauen, die seit 20 Jahren hier sind. Wenn die einfach Hausfrauen waren, nie Deutsch gelernt haben und bekommen die Möglichkeit nicht. ... Je mehr Möglichkeiten der Staat bietet, umso mehr ... können die Frauen das auch nutzen. Wenn Kindergartenplätze, mehr Kindergartenplätze, kostenlose Kindergartenplätze angeboten werden, natürlich können die Frauen auch arbeiten. Und wollen auch. ... Die, die jetzt gekommen sind seit 2015 haben die Information, dass das letzte Jahr Kindergarten gratis ist. Die können dann benutzen, die können arbeiten gehen. Die freuen sich schon. " (E4)

Hinsichtlich der Vermittlung von Frauen besteht eine spezielle Problemsituation bei den sehr jungen Frauen im Alter zwischen 17 und 21 Jahren, die ihre Schulbildung nicht in Österreich absolviert haben, sowie bei den Älteren ab dem 50. Lebensjahr, die aufgrund ihrer Altersgruppenzugehörigkeit per se eine Problemgruppe auf dem Arbeitsmarkt repräsentieren. Trotz hoher Arbeitsmotivation dieser Frauen existiert kein adäquates Stellenangebot.

„Ich hab' zwei, also ich hab eher ganz junge Frauen, die eher so zwischen 17 und 21 sind, die beim AMS Jugendliche sind, oder Frauen ab 40, also 50 und plus. Und das sind genau die zwei Gruppen, wo wir auch in Gesamtösterreich Schwierigkeiten haben, was Arbeitsplatzvermittlung geht. Weil die 50-plus kommen zu mir, wenn es um gesundheitliche Geschichten geht .... Und die ganz jungen, die zwar keine Schule in Österreich besucht haben, aber möchten irgendeine Ausbildung oder so ... “(E8)

\section{Berufliche Integration und Wertewandel}

Die berufliche Integration repräsentiert einen wichtigen Faktor im Kontext des Wertewandels. Die Einbettung in ein europäisch-säkulares Wertesystem ist für Personen in höherem Lebensalter schwieriger als für Jüngere. Kann eine Integration in den Arbeitsmarkt erfolgen, so erleichtert diese als „learning by doing“ die Involvierung in ein soziales Netz auf dem Arbeitsplatz und somit auch das Kennenlernen westlicher Werte.

„Also ich denke mir, es ist schwer, wenn jemand mit 50 Jahren nach Österreich auswandert, ihm die wirklichen Werte der Demokratie beizubringen ... vor allem, 
wenn man nicht in der Arbeitswelt Fuß fassen kann oder nicht sehr viele Menschen hat und diesen Diskussionsrahmen hat, sehr schwer verstehen, als die Kinder, die dann hier aufwachsen, oder Jugendliche, die hier aufwachsen in den Systemen. ... Man erlebt ja diese politischen Systeme, indem man ein Teil des Systems wird. ... Sie erleben auch am Arbeitsplatz, sie erleben das mit den Freunden, überall. Und dann wissen sie, wo die Werte sind. " (E8)

Ältere Befragte tendieren in realistischer Einschätzung ihrer geringen Chancen dazu, den Wert der Erwerbsarbeit umzuinterpretieren. Sie geben sich mit geringqualifizierten Berufen zufrieden, um Einkommen zu lukrieren und ihren Kindern den beruflichen Aufstieg zu ermöglichen. Sie motivieren ihre Kinder zur Absolvierung guter Ausbildungen und raten vom raschen Eintritt in wenig prestigeträchtige Branchen ab. Der sozioökonomische Aufstieg in der Aufnahmegesellschaft wird auf diese Weise in die nächste Generation transferiert.

„I: Und würden Sie gerne was mit Elektromechanik machen, wenn sie könnten, oder weiterstudieren? B: Ja, will ich, aber jetzt ist zu spät. ... ich bin 50 Jahre alt, ... ich wollte nicht bei XX [Lebensmittelkette] anfangen, sondern ich wollte meine Richtung, Fach weiterlernen etwas, ja. Aber leider. ... ich habe drei Kinder und ich will nicht meine Kinder in XX [Lebensmittelkette] arbeiten, oder Putzfrau werden oder in andere kleine Job oder so - wie sagt man? - uninteressante Job zum Beispiel. " (I39, weiblich, *1967, seit 1999 in Ö.)

\subsubsection{Regionale Arbeitsmarktchancen}

Hinsichtlich der variierenden Arbeitsmarktchancen im urbanen bzw. ländlichen Raum wurden divergierende Einschätzungen abgegeben. Faktum ist, dass eine Palette an Maßnahmen existiert, die auch die Binnenmobilität im Sinne der Arbeitsaufnahme fördert, z.B. das Pilotprojekt „Überregionale Vermittlung“ der WKO, BMWFW, BMASK und des AMS sowie „FIT“ zur Vermittlung in den Pflegesektor und andere Maßnahmen. ${ }^{107}$ Im ländlichen Raum bestehen Integrationsmöglichkeiten auf dem Arbeitsmarkt vor allem in den prosperierenden Regionen mit ein- oder zweisaisonalem Tourismus und zahlreichen Jobofferten in der Gastronomie, kaum hingegen in peripheren, agrarisch strukturierten Abwanderungsregionen. Einerseits werden von Expert/inn/en die positiven Jobperspektiven in den Tourismusgemeinden Westösterreichs hervorgehoben, andererseits zeichnen sich die urbanen Agglomerationen Wien sowie der oberösterreichische Zentralraum um Linz und das Einzugsgebiet von Graz durch ein breites und vielfältiges Angebot auf den Arbeitsmärkten aus. Wo die Jobperspektiven wirklich besser sind, kann nicht generalisierend beantwortet werden:

„,... dass die Leute sich entgegen der Realität [lacht] eigentlich mehr Arbeitschancen erhoffen in einer großen Stadt als am Land, ... was man ja grad über Wien so pauschal überhaupt nicht sagen kann. Ja, weil du in Tourismusgebieten oder in

107 Vgl. $<$ http://www.ams.at/vbg/service-arbeitsuchende/arbeitsuche/konventionsfluechtlingesubsidiaer-schutzberechtigte> 
Oberösterreich oder in Salzburg oder auch in Vorarlberg eigentlich mehr offene Stellen hast als in Wien, grad im niedrigqualifizierten Bereich. “(E5)

Positiv hervorgehoben wird, dass in Wien im Vergleich mit den anderen Bundesländern viele Projekte für die Arbeitsmarktintegration und die (Höher-)qualifizierung der Geflüchteten gestartet wurden sowie als Alternative auch die Selbständigkeit besonders gefördert wird. ${ }^{108}$

„Also viele wollen direkt nach Wien kommen, teilweise weil viele Bundesländer wahrscheinlich denen das Gefühl geben und andererseits, weil sich in Wien sehr viel tut. Politisch auch sehr viel tut. Auch es gibt viele Projekte, auch vom Arbeitsmarktbereich." (E8)

Es wird von Expert/inn/en allerdings auch betont, dass die Arbeitsintegration in Wien schon aus quantitativer Perspektive besondere Schwierigkeiten bereitet, da der Zuzug aus anderen Bundesländern die Zahl der Stellensuchenden erhöht und die Konkurrenz auf dem Stellenmarkt verschärft. Ein grundsätzliches Hindernis für die Arbeitsmarktintegration, welches unabhängig vom räumlichen Kontext besteht, manifestiert sich im niedrigen formalen Bildungsniveau vieler Afghan/inn/en.

„Also das betrifft natürlich stärker je niedriger das Bildungsniveau, umso schwieriger der Eintritt in den Arbeitsmarkt. Also das sieht man ja. Also Leute mit nur Pflichtschulabschluss haben auch unter den Österreichern mit Abstand die höchste Arbeitslosenquote. Das wird sich auch nicht ändern. ... Das ist sicher ein großes Problem. Noch dazu in Wien, hast du halt durch den Zuzug aus den anderen Bundesländern, weil die die Mindestsicherung kürzen, hast du noch zusätzlich Druck auf den Arbeitsmarkt, auf den Wohnungsmarkt, natürlich auch bis zu einem gewissen Grad, aber ich glaube das ist managbar, im Bildungssystem. " (E5)

In einigen Interviews wird auch auf das Phänomen der Rückwanderung im Rahmen der Binnenmigration in Österreich hingewiesen. Es handelt sich hierbei um Geflüchtete, die nach Erhalt des Asylbescheids mit hohen Erwartungen nach Wien gezogen sind, ihre Meinung ändern und aufgrund der besseren Chancen auf dem Arbeitsmarkt in eines der Bundesländer zurückkehren, in welchem sie bereits gelebt hatten.

„,... junge Männer, die zwar nach Wien kommen und sich zum Beispiel eine Lehrstelle wünschen oder denken, dass sie eine Ausbildung haben - weil vielleicht die einzige Möglichkeit, die sie in Wien derzeit haben ist eine Baustellenarbeit ... Und dann gibt es ja Betriebe, vor allem Tourismusbereich, in der Gastronomie, ..., die eigentlich Leute suchen. Und das AMS-Wien arbeitet ja auch schon jetzt ... intensiver mit den Bundesländern. Wenn jemand bereit ist und sagt: ,Ich möchte, ich bin alleine, hab' keine Familie, keine Verpflichtungen, Hauptsache ich bekomme eine gute Ausbildung und guten Arbeitsplatz. 'Dass sie dann wieder zurückgehen. Dass sie bekommen dann eine Ausbildung zum Beispiel, eine Lehre

108 Vgl. $<$ http://www.vienna.at/weg-vom-ams-fluechtlinge-als-unternehmer-am-arbeitsmarkt $5613026>$. 
als Koch in Bad Ischl zum Beispiel. Und ... einige junge Männer, die mich dann anrufen und sagen: ,Ja, ich bin jetzt dort und hab ' eine Lehre und ich mach das. "“ (E8)

Analog zu anderen Arbeitnehmer/inne/n wird die Wohnsitzwahl in einem hohen Ausmaß von der Lokalisation des Arbeitsplatzes determiniert. So finden sich Beispiele für mehrere relativ kurzfristige Wechsel des Wohnsitzbundeslandes jeweils im Gefolge der Arbeitsplatzwechsel:

„, Als ich neu hier in Österreich angekommen bin, wurde ich von Traiskirchen nach Steiermark geschickt. In Steiermark habe ich in einem Heim gelebt. Dann hatte ich dort eine eigene Wohnung bis 2005. Dann nach 2005 bin ich nach Linz gezogen. ... Weil ich dort meine Arbeit verloren habe, bin ich dann nach Linz gezogen. Dann habe ich wieder eine Arbeit in Steiermark gefunden und habe meine Arbeit in Linz gekündigt. Dann habe ich im Jahre 2008 wieder eine Stelle in Wien gefunden und bin hierhergezogen. Aber ich bin mit all meinen Wohnorten zufrieden. " (I63, männlich, *1965, seit 2001 in Ö.)

Während die besseren Chancen, außerhalb Wiens eine ungelernte Beschäftigung zu finden, in einigen Interviews hervorgehoben wurden, wird nur selten das Argument der Relevanz der afghanischen Community als Grund für die Arbeitsplatz- und Wohnsitzwahl in Wien angesprochen.

„Es ist zurzeit ein bisschen, was Arbeit betrifft, glaube ich, außerhalb [auf dem Land] besser, nur viele wollen eigentlich da, damit die Community hier ein bisschen stärker ist, dass sie versuchen, hier zu arbeiten. Was ich weiß, eigentlich auf dem Land ist zurzeit ein bisschen besser. In manchen Großstädten, zum Beispiel in Graz oder in Linz glaube ich, da gibt es schon Stellen, wo man dann eigentlich leicht Arbeit findet. ... Keine Facharbeiter-, sondern normale Hilfsarbeiterjobs gibt es schon dort ein bisschen mehr als in Wien. In Wien ist ein bisschen zurzeit schwierig. " (E7)

\subsection{Bildung und Ausbildung}

\subsubsection{Empirischer Forschungsstand}

Formale Schulbildung und berufsspezifische Weiterbildung repräsentieren zentrale Voraussetzungen für die Arbeitsmarktintegration, da unterschiedliche Dimensionen der Integration zueinander in enger Wechselwirkung stehen (vgl. TITELBACH et al. 2013; Hosner et al. 2017). Bildung und das Erlernen der Landessprache determinieren unter anderem auch die Teilhabe am gesellschaftlichen Leben (vgl. Alam 2008, p. 36; Neuwirth 2005, 2007; Dustmann und Glitz 2011; Chiswick und Miller 2015). Trotz klarer Rechtsgrundlagen ${ }^{109}$ bestehen zahlreiche Barrieren, die bewirken,

109 Die EU-Richtlinie zur Festlegung von Normen für die Aufnahme von Personen, die internationalen Schutz beantragen, legt in Artikel 14 fest, dass die Mitgliedsstaaten ,minderjährigen 
dass Asylwerber/innen, Asylberechtigte und subsidiär Schutzberechtigte nur erschwert Zugang zu Bildung haben (STEINER et al. 2018). Das UNHCR (2013, p. 38) zählt eine Reihe von Faktoren auf, welche die Bildungschancen von Geflüchteten beeinflussen: fluchtbezogene Traumata, Orientierungsprobleme in der westlichen Gesellschaft infolge religiöser und kultureller Unterschiede (NeuwIRTH 2007), gesundheitliche Probleme (STUBNIG und LACKNER 2007) sowie Diskriminierungserfahrungen (SCHEIBER 2007). Spezielle Herausforderungen stellen die Anerkennung im Ausland erworbener Qualifikationen (BIFFL 2012), noch mehr aber fehlende Ausbildungsnachweise dar (DIE Presse 2019).

In Österreich existieren zwar detaillierte Daten zur Bildungsbeteiligung von Personen mit Migrationshintergrund im Allgemeinen, aber kaum zu Geflüchteten im Speziellen (vgl. StatistiK Austria 2015; STEINER et al. 2018). Hervorzuheben ist, dass Geflüchtete erhebliche Integrationsbereitschaft mitbringen, bestrebt sind Deutsch zu lernen und berufliche Qualifikationen zu erwerben (KOHLBACHER et al. 2017; BUBERENNSER et al. 2016). Ohne Kenntnisse der Landessprache können die Platzierung auf dem Arbeitsmarkt und in der Folge berufliche und soziale Inklusion kaum erfolgen (Alam 2008, p. 72; MAhLFLEISCH 2015; ExPERTENRAT FÜR INTEGRATION 2017). Allerdings stellt selbst hohe Lernbereitschaft keine Garantie für ein in der Folge rasches Fußfassen auf dem Arbeitsmarkt dar. In Anbetracht des laut Kompetenzchecks des AMS äußerst heterogenen Qualifikationsniveaus (vgl. ARBEITSMARKTSERVICE 2016), des Faktums, dass die Afghan/inn/en im Vergleich der Herkunftsgruppen im Durchschnitt das niedrigste Bildungsniveau mitbringen (50 \% keinen oder nur einen Grundschulabschluss) sowie des mangelnden ,Systemwissens“ ist davon auszugehen, dass die Integration noch Zeit in Anspruch nehmen wird (vgl. EXPERTENRAT FüR INTEGRATION 2017, p. 37).

Völlig unbestritten ist die Wichtigkeit des Erlernens der Landessprache für die Inklusion und Integration (EssER 2006; PLuTZAR 2010). Gerade für Asylberechtigte und subsidiär Schutzberechtigte mit geringer formaler Bildung oder ohne Alphabetisierung stellt das Erlernen der deutschen Sprache eine besondere Hürde dar (KuHN 2013). Hier kommt Freiwilligen eine wichtige Rolle zu. Vor allem Ehrenamtliche kompensierten während der „,refugee crisis“ die Defizite des überforderten Bildungssystems (vgl. EXPERTENRAT FÜR INTEGRATION 2017, p. 46) sowie Informationsdefizite über Bildungsoptionen. Die Wichtigkeit des Ehrenamtes resultiert auch aus dem Faktum, dass das Regelschulsystem für geflüchtete Personen nicht der passende Rahmen ist, denn viele haben aufgrund ihres Alters hierzu keinen Zugang. Von professioneller Seite haben hier auch Sozialarbeiter/innen und das AMS Erhebliches geleistet (FETz 2011b).

Geflüchtete scheitern häufig nicht an fehlender Bildungsbereitschaft, sondern an den institutionellen Hürden, räumlichen und logistischen Rahmenbedingungen des Bildungszugangs oder am finanziellen und institutionellen Druck, möglichst rasch eine bezahlte Beschäftigung annehmen zu müssen. Benötigte Bildungsangebote sind vor Kindern von Antragstellern und minderjährigen Antragstellern in ähnlicher Weise wie den eigenen Staatsangehörigen den Zugang zum Bildungssystem“" zu gewähren haben (MAYRHOFER o.J.). 
allem in kleineren Gemeinden nicht vorhanden, daher ist die Frage der Mobilität und der Finanzierung von Bildungsangeboten wichtig (STEINER et al. 2018). Resümierend sei aber festgestellt: „Nicht jede Aus- oder Weiterbildung garantiert automatisch einen [attraktiven] späteren Arbeitsplatz ... Dies sollte geflüchteten Personen bewusstgemacht und kommuniziert werden“" (EuropäISCHES Forum AlPBACH 2016, p. 28).

\subsubsection{Analyse der Interviews}

\subsubsection{Bildungsorientierung}

In der afghanischen Community ist ein hohes Ausmaß an Bildungsmotivation weit verbreitet. Die Integration in den Arbeitsmarkt und Gelderwerb sind primäre Motivationsfaktoren, um sich (Weiter-)Bildung anzueignen, aber Bildung wird auch per se als relevant erachtet. Die Wichtigkeit der Beherrschung der Landessprache wird durchwegs betont. Allerdings finden sich auch viele Statements, die die Schwierigkeiten beim Erlernen der deutschen Sprache hervorheben; dies vor allem seitens älterer Personen, solcher mit geringer formaler Bildung und Frauen mit Kinderbetreuungspflichten. Ein in zahlreichen Bildungsbiographien auftretendes Problem manifestiert sich in den langen Wartezeiten auf den Asylbescheid, die eine Fortsetzung vor der Flucht eingeschlagener Bildungswege verzögern.

„Die Angebote werden total gut angenommen, also ich halte von diesem Motto ..., man muss die Menschen zu allem zwingen, halte ich für einen völligen Unsinn. Die Leute sind total motiviert, sie wollen, sie sind engagiert. Sie wissen, dass das ein Zeitfenster in ihrem Leben ist, das sie nützen wollen und müssen und die Motivation ist total da. " (E10)

„Ich meine, Bildung ist bei den Afghanen überhaupt so ein ganz großer ... Also der Auftrag, den sie von den Familien haben, heißt nicht unbedingt-oder in vielen Fällen nicht unbedingt - Geld verdienen und Geld nach Hause schicken, sondern einmal auch Bildung zu akkumulieren. ... Schon um Geld zu verdienen, aber auch ... Es gibt schon sehr hohe Wertschätzung von Bildung, auch in den Familien, die schon länger hier sind. " (E2)

„Also ich erlebe schon jetzt, Kinder, die zwar keine Volksschule in Afghanistan besucht haben, in Österreich dann ... die letzte Volksschulklasse gehabt und dass sie mittlerweile schon in der Gymnasium-Oberstufe sitzen. Sie haben auch extrem viel nachgeholt ... und die interessieren sich mehr für berufsbildende höhere Schulen, weil sie dann auch schneller wahrscheinlich arbeiten möchten. " (E8)

„,Der Expertenrat hat auch gesagt: Der Wille ist groß, aber noch kein Garant dafür, dass es schnell gehen wird aus zweierlei Gründen: 1) Die strukturellen Gegebenheiten, dass mehr Aufholbedarf da ist. Jugendliche haben ja noch nie in einem funktionierenden Staat gelebt. Außerdem lange Fluchtwege, vorher im Iran usw. Wir sind eine, Schein-Gesellschaft' in dem Sinn, dass man für alles einen Schein braucht. Sehr stark formalisierter Arbeitsmarkt, wo auch die Ausbildung 
stark formalisiert ist, was eine Herausforderung für die Flüchtlinge darstellt. 2) Das Systemwissen ist nicht so da. 97\% wollen Deutsch lernen, 83\% der Afghanen wollen bald einen Beruf ausüben, aber nur knapp die Hälfte findet eine fehlende Schulbildung oder Berufsausbildung als ein Problem für den Einstieg in den Arbeitsmarkt. "(E13)

„Also ich kenn' einen jungen Mann ... und nach eineinhalb Jahren ist er im Gymnasium, macht Matura. Also das muss man einmal schaffen, dass sie die deutsche Sprache gar nicht können und dann sie kommen und sie lernen zuerst einmal das. Und dann bringen sie sich auf dieses Niveau, dass sie dann die Matura fertigmachen und dann studieren! ... Aber man muss so denken, dass sie nicht alle studieren hier. " (E7)

„,... ich glaub ' die allermeisten haben begriffen, dass es wichtig ist für sie, dass sie die Sprache so schnell wie möglich lernen müssen .... Bildung ... ich glaub' dieser Wert, der ist eh vorhanden. Ja, das muss man natürlich irgendwie in die richtige Richtung lenken, weil sie das System bei uns nicht kennen und die Möglichkeiten, die es gibt. Also ich merk' da oft ein bisserl eine Ungeduld. ... Integration im Bereich Arbeitsmarkt, Sprache, Bildung. Das ist, ich glaub', den allermeisten klar. " (E5)

\subsubsection{Bildungserwartungen: Prozess der Modifikation und Adaption}

In den Interviews spiegelt sich ein hohes Level an Erwartungen bezüglich des Bildungserwerbs in Österreich sowie der Verwertung angeeigneter Kenntnisse wider. Diese Erwartungen müssen an die Realität angepasst werden. Denn Qualifikationen werden in Österreich häufig nicht anerkannt, die baldige Aufnahme einer Erwerbsarbeit ist nötig. Erwartungen haben mitunter auch wenig bis gar nicht Gebildete, die sich erhoffen, ihre Bildungsbenachteiligung nun endlich aufholen zu können. Eine Beschäftigung wird aus finanziell-rationalen Erwägungen zur Finanzierung des eigenen Lebensunterhalts vor allem aber auch für „remittances“ an die Familie und aufgrund institutionellen Drucks (z.B. seitens des AMS) aufgenommen, womit die angestrebte höhere formale Bildung häufig zurückgestellt werden muss. Dies geschieht kaum jemals freiwillig und wird von den Befragten ex post unterschiedlich bewertet. Faktisch ist durch die gesetzlichen Rahmenbedingungen fast ausschließlich der Zugang sogenannten Mangelberufen möglich, in Bezug auf eine Lehrausbildung vor allem dann, wenn eine Firma nachweist, keinen inländischen Lehrling zu finden.

Eine problematische Phase folgt häufig nach den Deutschkursen bzw. dem Pflichtschulabschluss. Trotz ausgeprägter individueller Motivation, den Pflichtschulabschluss nachzuholen, stockt in einigen Fällen die weitere Bildungskarriere, da der Sprung in weiterführende Schulen nicht für alle leicht zu bewerkstelligen ist. An dieser wichtigen Schnittstelle kommt es häufiger auch zum Abbruch von Bildungslaufbahnen. Von Expert/inn/en wird hervorgehoben, dass der Übergang vom Bildungs- in das Beschäftigungssystem besonders problematisch ist und vielfach an den Besuch der Deutschkurse unrealistische Hoffnungen geknüpft sind. 
„Dann sind sie raus aus der Schule, dann ist natürlich die Frage: Was können sie jetzt machen? Da gibt's dann verschiedenste Basisbildungsangebote. Brückenkurse, das sind so Vorbereitungskurse für den Pflichtschulabschlusskurs, und dann gibt's Pflichtschulabschlusskurse bei der VHS zum Beispiel. Dort können sie einen Bildungsabschluss nachholen. Das ist eine Möglichkeit, aber das Problem dann für die Asylwerber, ja wohlgemerkt ist jetzt, dass sie keine Lehrstelle aufnehmen können beziehungsweise nur in sogenannten Mangellehrberufen. Das ist jedes Jahr eine neue Liste von relativ wenigen Berufen, wo man sich bewerben kann und genommen werden kann, unter der Voraussetzung, dass der Arbeitgeber oder der Lehrbetrieb eine Meldung ans AMS macht und sagt: ,Ok, ich finde keinen Inländer für diesen Lehrberuf. Ich brauch genau den. 'Und dann kriegen die das genehmigt. ... Also alle von den UMFs, viele haben das Ziel Pflichtschulabschluss. Die wollen das alle machen, sind auch ziemlich ungeduldig, weil sie natürlich viel aufholen müssen plus die Sprache lernen. ... Aber das ist für die schon mal ein großes Ziel und wenn sie das erreicht sehen, dann sind die natürlich ungeduldig: Wie geht's jetzt weiter? Und da sind wir dann sehr eingeschränkt in den Möglichkeiten, die wir haben. Wir können sie unter Umständen auf eine Abend-HTL oder Abend-HAK oder Abendgymnasium vermitteln, weil da gibt's auch ein bisschen ein Angebot in Wien. " (E5)

„,... in ihren Bildungsanstrengungen behindert werden, massivst! Weil es dazu kommt, dass selbst wenn sie Deutschkurse machen bis B1 oder so was und den Pflichtschulabschluss, was viele Jugendliche ja in dieser Zeit schaffen, dass dann nichts darauffolgen kann. " (E2)

Viele Befragte plan(t)en, in Österreich ein Studium zu absolvieren, aber Defizite bei den Deutschkenntnissen und die Notwendigkeit, eine bezahlte Arbeit aufzunehmen, machen es vielfach notwendig, von den gewünschten Studien Abstand zu nehmen. Aufgrund der erlebten Probleme mit der Realisierung eigener Bildungserwartungen werden diese oft in die nächste Generation „verschoben“.

„Die Möglichkeiten schauen nicht gut aus, ich weiß es. Mathematik würde ich studieren. "(I60)

„Also mein Ziel war es, Arzt zu werden. Damit ich anderen helfen kann. Aber das kann ich nicht, weil mein Deutsch noch nicht so weit ist, dass ich weitermachen kann. Ich habe auch drei Semester das Gymnasium besucht, dann habe ich aufgehört. Dann habe ich eine Lehre gefunden und habe schon damit angefangen. " (I17, männlich, *1997, seit 2013 in Ö.)

\subsubsection{Bildungsniveau}

Typisch für die afghanische Community ist das weitgehende Fehlen einer bildungsmäßigen Mitte. Die Befragten verfügen entweder über einen Universitätsabschluss oder (nahezu) keine Schulbildung. Dies unterscheidet sie von Geflüchteten aus Syrien, dem 
Irak oder dem Iran, von denen ein hoher Anteil Zeugnisse mittlerer bzw. höherer Schulen vorweisen kann (ARBEITSMARKTSERVICE 2016).

Das mitgebrachte Bildungsniveau hängt von zahlreichen Faktoren ab, wie etwa dem sozioökonomischen Status der Familie, einem urbanen Wohnort oder vom Zuwanderungszeitpunkt. Im Verlauf der Flüchtlingswellen hat sich das Bildungsniveau verschoben. So waren die Afghan/inn/en der 1970er Jahre gut ausgebildet und kamen häufig des Studiums wegen nach Europa. Nach der sowjetischen Okkupation von 1980 flüchteten eher westlich orientierte Intellektuelle, nach dem Sturz des sowjetfreundlichen Regimes und der Machtübernahme durch die Mudschahedin 1992 die säkularkommunistisch orientierte, ebenfalls eher gebildete Schicht. Der Exodus religiöser Minderheiten sowie jener Gebildeten, die der von den Taliban repräsentierten konservativen Religiosität distanziert gegenüberstanden, verstärkte sich mit der Errichtung des Islamischen Emirats Afghanistan 1996. Die Bildungsintegration all dieser Gruppen verlief durchwegs unproblematischer als bei jenen Jahrgängen, die während der TalibanHerrschaft keinen Zugang zu Bildung hatten und daher immense Bildungsdefizite akkumulierten. Die „refugee crisis“ ließ dann erstmals in großer Zahl Personen ohne oder mit geringer Schulbildung nach Europa aufbrechen. Dazu beigetragen hat auch die Situation der afghanischen Flüchtlinge im Iran, die von der Partizipation am staatlichen Schulwesen ausgeschlossen sind.

„, Also die Leute, die aus Afghanistan kommen, haben entweder ein wirklich gutes Bildungsniveau, haben vielleicht studiert oder haben zumindest Matura, sprechen Englisch ... oder gar nichts. Also so in der Mitte gibt's da relativ wenig und es ist ziemlich wurscht, ob das jetzt Hazara oder Paschtunen sind .... Also da gibt's keinen Unterschied. "(E5)

„,... es gibt leider keine genauen Zahlen dazu, aber man kann so schätzungsweise sagen, o.k., zwei Drittel der Afghanen, die nach Österreich kommen, sind vielleicht Analphabeten oder haben kein höheres Bildungsniveau. Das kann auch stimmen, weil ich kenn ' das auch aus der persönlichen Erfahrung, dass man Menschen hier in der Beratung sieht und wenn wir bestimmte Übersetzungen vorlegen und sagen: ,Können Sie das lesen?', dann kommt auch die Antwort: ,Nein!'. Sie können nicht lesen. Und das zeigt eben, dass sie keine Schule besucht haben oder besuchen konnten. Weil einfach in einem Land, wenn Krieg herrscht, wird alles zerstört. " (E6)

\subsubsection{Wechselwirkungen mit anderen Feldern struktureller Integration}

Der State of the Art der Analysen in Österreich dokumentiert, dass die formale Bildung den Integrationsverlauf maßgeblich beeinflusst (KUHN 2013; HosNER et al. 2017; Manahl 2017). Laut Expert/inn/en besteht ein Konnex zwischen Bildung und jenen Kompetenzen, die die Integration erleichtern. Gebildete sind flexibler und finden sich im Umgang mit Institutionen eher zurecht. Ihre Systemintegration verläuft rascher und konfliktfreier. Dies wirkt sich auch positiv auf ihre soziale Integration aus. 
Personen, die muttersprachlich nicht alphabetisiert wurden, tun sich am schwersten mit dem Erlernen des Deutschen. Die Absolvierung des Pflichtschulabschlusses stellt für nicht wenige Geflüchtete eine große Herausforderung dar. Valide Prognosen zum Verlauf der Bildungsbiographie für jene, die vor ihrer Flucht noch keine Schulbildung genossen, zu stellen ist schwierig, da Langzeitstudien fehlen.

„,... die Arbeitsmarktintegration, das ist wirklich mit den Afghanen glaub'ich die größte Herausforderung, weil viele halt einen sehr niedrigen Bildungsabschluss haben, manche gar keinen. Viele der Afghanen, die nach Österreich gekommen sind, kommen eigentlich aus dem Iran und nicht direkt aus Afghanistan und die waren ja dort auch ausgegrenzt oder zumindest hatten keinen einfachen Zugang zum staatlichen Bildungssystem. Dadurch kommen die relativ oft zu uns und sind in Dari oder Farsi halt wacklig oder gar nicht alphabetisiert. Und je höher der Bildungsgrad ist, umso leichter finden sich die Leute natürlich zurecht. Die brauchen dann weniger Hilfe. Wenn jetzt jemand kommt mit einem Universitätsabschluss, den schickt's halt irgendwohin, der macht das schon. Also um den braucht man sich da irgendwie nicht so viele Sorgen machen. " (E5)

„Also ich habe selber einen guten Bekannten, ... Mitte 20 ist er gekommen und jetzt ... ich glaube nach sieben oder acht Jahren hat er mit 31 Jahren endlich den Pflichtschulabschluss geschafft. Und das ist-glaube ich-kein Einzelfall, die es ganz schwer haben, das zu schaffen. " (E2)

Personen ohne formale Bildung werden mit einer Unzahl an Problemen konfrontiert. Eine gewisse Kompensation bieten die Wertekurse, die praktische Informationen und Orientierungsmöglichkeiten darüber, wie „Österreich im Alltag funktioniert“ und welche Institutionen und Systeme existieren und wie sie genutzt werden können, beinhalten. Darüber hinaus ist das zusätzliche Beratungsangebot der Beratungsstellen und Vereine wichtig, die integrationsrelevante Informationen zum Bildungssystem in Österreich anbieten. Die Qualität der Angebote ist jedoch laut Expert/inn/en manchmal umstritten.

„Das ganze österreichische Schulsystem und Bildungssystem, wie das aufgebaut ist, ... Das ist ja umso schwieriger für Personen, die dann einfach ... in Österreich leben, die Sprache nicht können, da in der Bildungsschiene mal Fuß zu fassen. Mittlerweile hat sich sehr viel getan, ... Es ist halt aber eine sehr, sehr große... Hürde, auch für den Arbeitsmarkt, diese Situationen zu lösen. “(E8)

„,Aufjeden Fall, Deutsch zu lernen, auch für Analphabeten gibt es die Möglichkeit, indem sie Alphabetisierungskurse besuchen und dann weiterführende Kurse ... Wertekurse ..., das der ÖIF österreichweit anbietet, ... ist auch eine Basis, für Menschen, die neu in Österreich sind, dass sie auch einen Gesamtüberblick haben, wie man in Österreich sein Leben starten kann. So was hat mir zum Beispiel vor 15 Jahren gefehlt. Also ich musste immer da und dort Leute fragen: Was mache ich, wenn ich beim Gesundheitsthema eine Frage habe oder irgendwas nicht weiß? 
Wie muss ich mich bei Arbeitsamt melden? Wie muss ich mich verhalten, wenn ein Österreicher ... Bei diesem Wertekurs, in acht Stunden wird einfach ein großer Überblick gegeben, ... O.k., die Gebildeten wissen das vielleicht, aber die weniger Gebildeten oder Analphabeten wissen das nicht. Und damit sie das wissen, eben der Wertekurs hilft ihnen. “(E6)

„Also Bildung, da können die Vereine schon was helfen. Das ist auch eines der Dinge, die diese Interessengemeinschaft der afghanischen Schüler/innen und Studierenden massiv macht, Vorträge zum österreichischen Bildungssystem ... In anderen Bereichen, also zum Beispiel was Rechtsberatung und so anbelangt, das funktioniert gar nicht. " (E2)

Wie bei der formalen Schulbildung divergieren auch die Levels berufsbezogener Ausbildung. Damit hängen aber auch die Möglichkeiten des Transfers mitgebrachter Kenntnisse, z.B. in der Baubranche, zusammen. Während z.B. Baufacharbeiter aus dem wirtschaftlich besser entwickelten Iran bereits nach kurzen Einschulungen bei österreichischen Baufirmen im Einsatz sein können, ist die Integration ehemals in Afghanistan tätiger Facharbeiter schwieriger, da sie ihr „training on the job“ anhand sehr veralteter Techniken genossen. Defizite bestehen auch bei ehemals in der Landwirtschaft tätigen Menschen, welche viele Qualifikationen, die in der technisierten Agrarproduktion Österreichs gefordert werden, nicht aufweisen.

„,Aber ein Unterschied ist, wenn ein Bauer oder nehmen wir einen Tischler in Afghanistan gewesen ist; mit welchen Materialien er gearbeitet hat und so. Also da sind Riesenunterschiede auch in den Entwicklungs- und Arbeitsprozessen. Viele, die im Iran gearbeitet haben oder als Hilfsarbeiter, ... die kennen sich viel besser aus mit den Materialien und so, aber natürlich Iran hat auch Fortschritte gemacht in den Bereichen. Und wenn sie im ... Baubereich und grad in der Industrie, werden die Menschen, die aus dem Iran kommen, sie werden auch sofort bereits eingestellt. Sie tun sich nicht schwer. Die lernen ein bisschen die Sprache, aber Arbeitsmethoden, Technik, Maschinen kennen sie.... Also Afghanistan hat ja kaum Industrie, ... natürlich hat man sich dann in diesen Bereichen gar nicht entwickeln können. “(E8)

„,Aber ich arbeite. Gefällt mir in der Baustelle, weil ich kenne das alles. Kann ich schon arbeiten, kann ich, aber im Iran habe ich so lange gearbeitet, aber leider keine Zertifikate oder so etwas noch. Aber hier muss ich lernen. Zuerst lernen und dann arbeiten. “ (I25, männlich, *1997, seit 2014 in Ö.)

„Die Bildungsdrehscheibe ist so eine Art Mini-AMS für Asylwerber. Also wir erheben halt Kompetenzen, Bildungsbedarf in verschiedenen Bereichen, von Sprache über Praktika, Basisbildungskurse. Je nachdem, ... was die Leute halt brauchen. "(E5) 


\subsubsection{Abwertung von Bildungsabschlüssen}

Die Abwertung formaler Bildung sowie berufliche Dequalifikation stellen ein in Bildungs- und Berufsbiographien von Geflüchteten häufig auftretendes Phänomen dar. Dies gilt auch für Qualifikationen in Mangelberufen, da diese nicht eins zu eins auf die österreichischen Verhältnisse übertragbar sind. So sind trotz eines Mangels an Allgemeinmediziner/inne/n im ländlichen Raum Österreichs die bürokratischen Hürden der Nostrifikation von Universitätsabschlüssen erheblich. Die meist als temporär betrachtete Annahme einer dequalifizierenden Beschäftigung ist riskant. Das lukrierte Einkommen ist zwar erwünscht, der zeitliche Aufwand der beruflichen Tätigkeit verhindert aber häufig eine Fortsetzung der ursprünglich intendierten Bildungslaufbahn.

„... da stellt sich bei uns vor allem das Thema Nostrifizierung. Wie läuft die Anerkennung der Ausbildungen, die sie in den Herkunftsländern realisiert haben? Da haben wir extreme Schwierigkeiten, was die Zeit, was die Finanzierbarkeit betrifft, auch was die berufsspezifische Sprachausbildung betrifft ... “(E10)

„, Und mein Problem, dass ich gedanklich immer daran hing, unbedingt also ein bisschen eher intellektuell tätig zu sein und vielleicht die fertiggestellte AufgabeStichwort: meine Dissertation - zu Ende bringen. Ich hatte nur mehr eine kürzere Zeit, damit ich mit dem fertig werde. Aber das wurde von Behörden in Österreich nicht akzeptiert. Die haben eigentlich von Anfang an von mir verlangt, dass ich unbedingt arbeiten muss .... Egal was, ich musste arbeiten. Und das ist auch der Grund, warum ich nicht weiter studieren konnte. Ich habe aufgehört. Dann habe ich den Job angenommen. Und natürlich, wenn man einen anderen Job hat, total in eine andere Richtung, dann kann man das nicht machen, was man ursprünglich geplant hat. " (I37, männlich, *1952, seit 1988 in Ö.)

\subsubsection{Bildungsintegration: Herausforderungen und Barrieren}

Relevanz des Erlernens der deutschen Sprache

Von allen interviewten Personen wird die Wichtigkeit des Erlernens der deutschen Sprache betont. Dies deckt sich mit der Bedeutung, welche der Sprache in allen Integrationsmaßnahmen beigemessen wird. Das Erlernen des Deutschen repräsentiert einen wichtigen Schritt, um am österreichischen Bildungssystem und weiterführenden Bildungsangeboten partizipieren zu können. Bei allen Befragten haben Bemühungen zum Spracherwerb stattgefunden.

„Es war für mich sehr wichtig, dass ich die Sprache lerne. Aber aufgrund von einigen Problemen, habe ich mir sehr wenig Zeit gelassen damit, und es war nicht so, wie ich es mir erhofft hatte. Ich bin der Meinung, dass für ein besseres Leben hier, ist die Sprache der Schlüssel dafür. Und deswegen gebe ich umso mehr dafür, die Sprache zu lernen. “ (I48, männlich, *1991, seit 2014 in Ö.) 
„Im Allgemeinen ist die deutsche Sprache sehr schwierig, aber wir leben hier, deshalb müssen wir auch die Sprache lernen. “ (I55, männlich, *1998, seit 2014 in Ö.)

\section{Deutscherwerb in Kursen}

Die Problematik der Deutschkurse ist vielschichtig. Sehr häufig wurde Kritik bezüglich der langen Wartezeiten auf den Asylbescheid und auf geeignete Kurse verbalisiert. Dies betrifft primär den institutionellen Zugang. Andere waren mit der Qualität der Kurse nicht zufrieden. Sie kritisierten vor allem, dass diese zu selten und in zu geringer Stundenzahl angeboten wurden, um wirklich nachhaltige Lernerfolge erzielen zu können. Dies galt aber durchwegs für das Angebot in kleineren Gemeinden, wo Geflüchtete zusätzlich mit Problemen der Erreichbarkeit und beträchtlichem Fahrtaufwand konfrontiert waren. Zum Teil wird der Nachweis des Besuchs der Deutschkurse irrigerweise als Entscheidungsfaktor im Asylverfahren interpretiert. Seitens der Respondent/inn/ en wird die in den Deutschkursen erreichbare Sprachbeherrschung realistisch eingeschätzt. Alle betonen, dass Geläufigkeit erst in der Kommunikation mit Einheimischen erworben wird, da diese ,... takes place in 'real life', which makes social contacts crucial” (UNHCR 2013, p. 37). Anhand der Expert/inn/eninterviews wird deutlich, dass sich mit Bewältigung der „refugee crisis“ viele positive Veränderungen bezüglich des Zugangs zu Deutschkursen, des Bildungsangebots generell und der Beratungsleistungen des AMS vollzogen.

„Na, es hat sich verbessert, ... Ich habe das oft von den jungen Burschen gehört, die ... sagen: ,Wir haben keine Beschäftigung. Wir haben Deutschkurs einmal in der Woche zwei Stunden, das bringt uns gar nichts. .... Ich lern dort gar nichts. ' Und die lassen sich gehen dann. ... Dann passiert es, dass sie überhaupt keine Lust zu gar nichts mehr haben. Sagt er: ,Ich verzichte auf diesen Deutschkurs auch, dann schlaf" ich lieber. "“(E4)

„, Wobei jetzt in den letzten zwei Jahren vom AMS doch mehr getan wird, auch bildungsmäßig. Also zumindest in Wien [ist] die Situation besser geworden. “ (E2)

„Und dass sie generell also an Bildung leiden. Also Bildung fehlt bei ihnen, wie gesagt. Sprachförderung wurde angesprochen, betrifft aber nicht Asylberechtige und subsidiär Schutzberechtigte, weil für diese zwei Zielgruppen gibt es eben jetzt genügend Fördermittel und Fördermöglichkeiten. Wenn sie diese nutzen, natürlich können sie auch integriert werden ... Und heutzutage Flüchtlinge können von Alphabetisierung beginnen bis A1 bis A2 bis B1 und B2. Das heißt, es gibt schon die Möglichkeit. Wenn man diese nutzt, dann kann man auch integriert werden. " (E6)

„, Aber in Österreich am ersten Tag wir haben die Möglichkeit gehabt. Zum Beispiel in Bad Kreuzen gibt es nicht einen Deutschkurs, aber Deutsch zu lernen, gibt es 
eine Möglichkeit und in Traiskirchen auch gibt es eine Möglichkeit Deutsch zu lernen. “ (I26, männlich, *1974, seit 2013 in Ö.)

„In irgendeinem Dorf da brauchte ich eine halbe Stunde bis zu einem Zug und halbe Stunde bis Hauptstadt und halbe Stunde bis irgendwo einen Kurs oder so zu besuchen. Das heißt, wir hatten jeden Tag eine halbe Stunde Kurs, OK, aber ich war fünf Stunden pro Tag unterwegs. Also eineinhalb, eineinhalb hat sicher gedauert. " (I11, männlich, *1988, seit 2011 in Ö.)

Die Absolvierung der Kurse stellt keine Garantie für ausreichende Sprachkompetenz dar, da das mitgebrachte Bildungsniveau, unterschiedliche Lerngeschwindigkeiten und persönliche Talente zum Erlernen von Sprachen in den Kursen kaum berücksichtigt werden können. Es wurden auch Klagen darüber verbalisiert, dass seitens des AMS der Besuch gewünschter Kurse nicht bewilligt wurde und auch die Leistbarkeit der Kurse stellt eine Hürde dar.

„,Mein größtes Problem ist, ich kann nicht sprechen Deutsch ... Und ich ... viele Male AMS gehabt. Ich bin gegangen und gesagt: ,Bitte geben mir B1-Kurs wieder . Aber er hat gesagt: ,Nein, das ist nicht möglich, ich muss arbeiten '... bis jetzt ich habe keine B1-Kurs bekommen, Sprachkurs nicht bekommen und Arbeit auch. Ich habe keine Arbeit. " (I19, weiblich, *1973, seit 2011 in Ö.)

Deutscherwerb auf Eigeninitiative, mittels , learning on the job “ und sozialer Integration

Es wird auch die Wichtigkeit der Eigeninitiative hervorgehoben. Die in den Kursen vermittelten Kenntnisse können nur eine Basis bereitstellen, Fortschritte müssen durch eigene Anstrengungen und Kommunikation erzielt werden. Eine wichtige Möglichkeit zur Kommunikation ist der Arbeitsplatz, des Weiteren wird auch das Internet, z.B. über das Videoportal YouTube, zum Lernen genutzt.

„, Und ich habe den Job im ersten Monat ... Das war wirklich, wirklich schwer. Wegen der Sprache ... Und dort kannst du nicht ruhig bleiben. Du musst immer mit den Mitarbeitern reden: 'Das musst du so machen, das musst du so machen.' ... Aber es hat mir wirklich gut geholfen. Jetzt kann ich fast fließend sprechen. " (I1, männlich, *1989, seit 2013 in Ö.)

„... ich suche immer im YouTube diese Dialoge von Deutsch. Ich möchte damit meine Sprache besser werden. ... Ja, Deutsch ist für mich sehr richtig. Wenn ich Deutsch lerne, dann kein Problem für mich. " (I19, weiblich, *1973, seit 2011 in Ö.)

Von Expert/inn/en wird auf die Problematik des Deutschlernens in Kursen mit beschränkter Praxisorientierung hingewiesen. Für wichtig halten sie die Einbindung des Deutschlernens in das Erlernen eines Berufs sowie die Verbesserung der Sprachgeläufigkeit durch die Kommunikation am Arbeitsplatz. Die Kombination von Arbeitstrai- 
nings und berufsbezogener Ausbildung mit Deutschförderung erfüllt auch den Zweck einer rascheren sozialen Integration.

„Ja, man sollte die Afghanen mehr ausbilden, mehr Fachausbildung anbieten. ... es gibt auch Fachschulen, wo sie im Laufe der Zeit auch ihr Deutsch verbessern können. Sie können früher anfangen mit Praxis und Theorie. Zum Beispiel, wenn sie auf A2-Niveau sind, können sie gleich mit einem Facharbeiterkurs Schweißer zu lernen beginnen. Gleichzeitig aber nicht nur Schweißer lernen, sondern damit auch die deutsche Sprache. " (E3)

„Das AMS hat da viel mehr Möglichkeiten, was zu machen. Die stecken die Leute halt in Kurse, aber für sie sinnvoller ist ab einem gewissen Deutschniveau, dass die Leute dann wirklich Arbeitstrainings bekommen, eine Nachqualifizierung bekommen. Weil sozusagen die gesellschaftliche Integration funktioniert ohnehin am stärksten über den Arbeitsmarkt. Das ist klar. “ (E5)

Die Beherrschung der Landessprache und soziale Integration stehen gemäß Forschung in einem engen Interdependenzverhältnis, was sich im Sample sowohl in Statements der Expert/inn/en widerspiegelt als auch den Einschätzungen vieler Respondent/ inn/en entspricht. Befragte, die offen sind für Interaktionen mit Deutsch sprechenden Personen, verbessern damit sukzessive ihre Sprachkompetenz und etablieren damit ein tragfähiges soziales Netz, welches ihnen auch im Rahmen ihrer diversen Bemühungen im Rahmen der strukturellen Integration zugutekommt.

„,... wenn man jetzt zum Beispiel nur rein ... die Sprachkenntnisse sich anschaut. Natürlich, je mehr und intensiver ein Kontakt zu ... ich will jetzt nicht sagen Österreichern, sondern halt einfach alle Leute, die deutschsprachig oder muttersprachlich oder gut Deutsch sprechen, desto besser sprechen die natürlich Deutsch. “(E5)

„,Die Sprache habe ich nicht durch einen Deutschkurs gelernt. Nein, ich habe es viel mehr durch die Kommunikation mit Leuten gelernt. Ich habe manchmal sogar alte Leute auf der Straße angehalten, um mit ihnen Deutsch sprechen zu können. Immer wenn ich die Wohnung verlassen habe, sei es um spazieren zu gehen, oder um trainieren zu gehen, habe ich immer versucht, mit Leuten in Kontakt zu kommen, bis ich dann immer besser wurde. Großteils habe ich die Sprache auch in der Arbeit gelernt. " (I63, männlich, *1965, seit 2001 in Ö.)

Das Level der Deutschkenntnisse beeinflusst den Integrationsverlauf, da dies auch das persönliche Gefühl fördert, in der österreichischen Gesellschaft angekommen zu sein und einen Teil dieser Gesellschaft zu bilden. Dieses Bewusstsein steigt mit der Geläufigkeit der Landessprache an, benötigt aber vor allem Zeit.

„Ich habe das Gefühl, ein Teil dieser Gesellschaft zu sein, aber noch nicht 100\%, vielleicht 50\%. Ich kann jetzt leicht mit den Menschen kommunizieren und fühle mich auch als Teil dieser Gesellschaft. Aber früher, wie ich die Sprache noch nicht gut genug konnte, fühlte ich mich fremd. Ich meine, wenn ich irgendwohin wollte 
und Fragen hatte, konnte ich nicht leicht meine Antworten erhalten. Aber jetzt kann ich es. " (I48, männlich, *1991, seit 2014 in Ö.)

Die bereits in früheren Dekaden nach Österreich migrierten Afghan/inn/en können als Kontrollgruppe herangezogen werden. Von ihnen wird durchwegs die Sprache als Schlüssel zur Integration in Österreich hervorgehoben und die Wichtigkeit des Zeitfaktors betont. Ein Nachteil war, dass es früher ein geringes Angebot an Deutschkursen gab und man Kurse auf eigene Kosten besuchen musste. Im Vergleich zur Zeit ihrer Zuwanderung, als man ihnen vorurteilsfrei begegnete, stellen sie aber zunehmend auch sprachliche Integrationshürden infolge einer steigenden Verschlossenheit der Bevölkerung ohne Migrationshintergrund bis hin zur Xenophobie fest. Mit dieser zunehmenden Ablehnung werden nicht nur Erwachsene, sondern auch bereits Kinder im Schulalter konfrontiert.

Das Deutschlernen bildet bei drei Gruppen eine Barriere: 1) bei Älteren, 2) jenen mit geringem Bildungsniveau und 3) familiär beanspruchten Frauen. Es handelt sich hierbei um dieselben Personen, denen die Partizipation an Bildungsangeboten generell sowie auch das Fußfassen auf dem Arbeitsmarkt erhebliche Probleme bereitet.

„Für mich war es sehr schwierig. Bis jetzt habe ich es nicht geschafft, die Sprache zu lernen. Es ist jetzt mittlerweile Jahre her. Russisch kann ich sehr gut, aber Deutsch nicht. Meine Tochter hat es sehr schnell gelernt und innerhalb von einem Jahr konnte sie sehr gut die Sprache, wie die Österreicher/innen. ... Im jüngeren Alter ist das Erlernen leichter ... “ (I28, weiblich, *1977, seit 2013 in Ö.)

\section{Einfluss von Gender Role Models}

Im Sample berichtete zwar keine Frau über gravierende Probleme mit ihrem Ehemann in Bezug auf ihre Teilnahme an Bildungsangeboten, aber die Einschätzungen der Expert/inn/en hinsichtlich der Auswirkungen geschlechtsspezifischer Rollenmuster auf die Partizipation an Bildungsangeboten, an Deutschkursen im Speziellen und bezüglich der Verwertungsmöglichkeiten erworbener Bildung divergieren. Einige Expert/inn/en erwähnen sehr wohl Probleme mit konservativ-traditionell orientierten Männern, andere sehen nur wenig Konfliktpotential. Eine Vielzahl an soziodemographischen und sozioökonomischen Determinanten, wie Alter, Bildungsniveau, Sozialschichtzugehörigkeit, aber auch individuelle Charakteristika wie konservative Wertorientierungen, Vertrautheit mit westlichen Normensystemen, das persönliche Toleranzlevel, Selbstbewusstsein und Durchsetzungsvermögen etc. spielen eine Rolle.

„,... also der Hauptgrund war ein Nähkurs. Alphabetisierung war daneben. Und es ist natürlich auch um Empowerment der Frauen gegangen. Das hätten die Männer nicht erlaubt, ... Dass eben das dann manche Männer ablehnen. Also man kann das jetzt auch nicht pauschalieren. " (E1)

„Und da die Frauen zum Teil eben sehr ambitioniert sind, die kommen. Also die sagen: ,Ok, ich will in die Schule gehen! Ich will studieren!", was manchmal die Männer ein bisschen überfordert. “(E2) 
„,... jetzt nur mit den Leuten, mit denen ich zu tun habe, mir noch nie zu Ohren gekommen ist, dass irgendein Afghane sagt: ,Ja, meine Tochter oder meine Frau soll nicht den Deutschkurs machen oder soll nicht in die Schule gehen oder soll keine Bildung bekommen. 'Also ich glaub' das eher weniger. “(E5)

Das Bildungsangebot in Österreich und die Teilnehmenden stehen in einem dynamischen Interaktionsgeflecht. Religiosität kann nicht monokausal der primäre Erklärungsfaktor für die Nichtpartizipation an Bildungsangeboten sein, denn:

„Ich habe oft Leute gehabt, die vollen Hijab hatten und trotzdem bei mir in der Beratung waren und wollten in einen Deutschkurs und auch andere Maßnahmen besuchen. Also ich würde das nicht mit jetzt grad der Religion und Überzeugung oder so verbinden, sondern eher wenn, dann nur mit Alter und mit Bildungsniveau. Ein in zahlreichen Bildungsbiographien auftretendes Problem manifestiert sich in den langen Wartezeiten auf den Asylbescheid, die allzu oft eine Fortsetzung eingeschlagener Bildungswege verhindern. " (E6)

Eine spezielle Situation liegt in Kursen vor, in denen eine Männermajorität besteht, was manchem Ehemann missfällt. Diese Situation kann auch bei Frauen, die an koedukative Strukturen nicht gewöhnt sind, Unbehagen hervorrufen:

„Wo sie manchmal schon ein Problem damit haben ist, ... wenn du jetzt einmal einen Deutschkurs bei uns hast, da sitzen zehn Männer und eine Frau oder zwei Frauen drin, ... also das wollen manche nicht. “ (E5)

„Also in den Wertekursen die Frauen sagen selten was, bei den Afghanen ... und haben irgendwie selten einen Beitrag gebracht, sondern geredet haben eigentlich immer nur die Männer. " (E5)

Es ist hervorzuheben, dass vor allem die Frauen den Wert von Deutschkenntnissen und höherwertigen Ausbildungen erkennen und bestrebt sind, das Angebot zu frequentieren. Eine wesentliche Hürde manifestiert sich in Kinderbetreuungsverpflichtungen. Viele Frauen entwickeln mit längerer Dauer ihres Aufenthaltes in Österreich immer mehr Aktivitäten in Richtung Weiterbildung, Ehemänner sind zumeist damit einverstanden und übernehmen temporär auch die Kinderbeaufsichtigung.

„Sie sehen es halt wirklich als enorme Chance auch als Frauen zu Bildung zu kommen und irgendwie da auch nicht so durch traditionelle Strukturen behindert zu werden, wie es halt im Herkunftskontext ist. Da habe ich schon den Eindruck. “ (E2)

„Das ist schon ein Problem, wie viele Afghaninnen in Deutschkurse oder Alphabetisierungskurse gehen können, wenn sie kleine Kinder haben. Das heißt, diese Kurse müssen eigentlich so organisiert sein, dass auch sichergestellt ist, dass während die Mutter Deutsch lernt oder einen Alphabetisierungskurs macht, es auch eine Kinderbetreuung gibt. " (E1) 


\section{Ältere Geflüchtete}

Ältere haben generell mehr Probleme, mit den Herausforderungen der Situation nach der Flucht umzugehen und beim Erlernen neuer Inhalte. Zwar wird viel Bildungsund Arbeitsmotivation auch von Befragten höheren Alters artikuliert, sie schätzen ihre limitierten Chancen aber durchwegs realistisch ein. Expert/inn/en berichten, dass ältere und wenig gebildete Personen generell im strukturellen Integrationsprozess mit zusätzlichen Herausforderungen konfrontiert werden. Dazu kommt eine ohnehin langfristig hohe Rate an Langzeitarbeitslosigkeit auch bei den Inländer/inne/n im Alter von über 50 Jahren.

„Schwierig wird's dann bei den Leuten, die ... älter sind, wenig bis keine Schulbildung haben, egal aus welchem Land sie sind. Also, dass die dann einen Fuß in die Tür kriegen am Arbeitsmarkt, das ist ein bisserl schwierig, dauert manchmal länger. Und der Ansatz ist eher der, wenn man sagt, die bringen Schulbildung mit oder bringen Berufserfahrung mit, dass man sagt: ,Ok - beim AMS - ja o.k., du gehst jetzt nicht Hilfsarbeiterjob, hackeln', sondern wir versuchen dich auf dein maximales Niveau zu ... zu qualifizieren. '... Das dauert zwar länger, aber macht mehr Sinn ... für die Person und auch volkswirtschaftich ... Also der Druck am Arbeitsmarkt ist in Wien auch grad im niedrigqualifizierten Bereich viel höher als bei Facharbeitern oder Akademikern. " (E5)

„Aber mit dem Alter ist es ein Problem. Die älteren Menschen, vor allem Ungebildete, haben es sehr schwierig zu verstehen. Also zum Beispiel es gibt bestimmte Arbeitsschritte, die man ihnen erklären muss. Also man sagt: ,Das und das und das. 'Obwohl du das in der Muttersprache gemacht hast, da merkst du schon die Person versteht nicht richtig. “ (E6)

\subsection{Soziale Beziehungen und Netzwerke}

„,... die haben mir das Gefühl gegeben, egal was ich brauche, sie sind da.“

\subsubsection{Einleitung}

Das folgende Kapitel widmet sich den facettenreichen sozialen Beziehungen und Netzwerken der Respondent/inn/en dieser Studie. Zunächst wird auf die Rolle sozialer Netzwerke und das daraus gewonnene soziale Kapital im Allgemeinen und dessen Stellenwert im Besonderen eingegangen. Im Weiteren werden drei Kategorien von Sozialkontakten genauer betrachtet: soziale Beziehungen zu Personen der österreichischen Mehrheitsgesellschaft, jene zu Personen der eigenen ethnischen/religiösen Community sowie solche zu Personen aus anderen Minderheiten in Österreich. Dabei wird jeweils auf den Zugang, den Nutzen sowie die möglichen Herausforderungen der Einbindung in das Netzwerk der jeweiligen Gruppe eingegangen. Abschließend werden soziale Kontakte als Basis für die Entwicklung eines Gefühls der Zugehörigkeit und die Integration in die österreichische Gesellschaft diskutiert. 


\subsubsection{Soziales Netzwerk - Soziales Kapital}

Individuen sind eingebunden in ein Netzwerk unterschiedlichster sozialer Beziehungen und können daher nicht isoliert voneinander betrachtet werden. Wie auch Williams in ihrer ethnographischen Studie zu sozialen Netzwerken Asylsuchender in Großbritannien betont, können Asylwerbende nicht als passive Unterstützungsempfänger/innen betrachtet werden, sondern zeigen sich als aktive, sich soziale Kontakte zu Nutze machende und Unterstützung suchende sowie leistende Akteur/inn/e/n (WiLliams 2006). Manche Beziehungen sind schwächer (weak ties), z.B. Kontakte in Vereinen oder mit Arbeitskolleg/inn/en, andere intensiver (strong ties), wie z.B. in der Familie oder mit Freunden. Sie alle stellen eine Verbindung zwischen unterschiedlichen Akteur/inn/en dar und ermöglichen es Individuen oder Kollektiven, Ressourcen aus dem Netzwerk zu nutzen (DeINDL 2005). Insbesondere in der Migrations- und Fluchtforschung erweist sich die Betrachtung von Netzwerken und der damit verbundenen Ressourcen als relevant.

Die Stärke des Netzwerkansatzes liegt darin, dass er eine Verbindung zwischen der individuellen Ebene (Mikro-) und übergeordneten Strukturen (Makro) ermöglicht (LIN 2001). Wir gehen dabei nicht von geographisch abgesteckten Einheiten aus, sondern von Beziehungen zwischen Menschen, wodurch sich eine Perspektive eröffnet, die sich zeitlichen und räumlichen Veränderungen, z.B. durch Migration, anpasst (BOYD und NowAK 2012). Anstatt Migrant/inn/en als isolierte Akteur/inn/e/n zu verstehen, hilft die Analyse von Netzwerken dabei, sowohl ,ties“ zwischen Migrant/inn/en und Personen in Herkunfts- und Aufnahmeländern zu erkennen als auch den daraus entstehenden Nutzen dieser Ressourcen zu analysieren (BoyD und NowaK 2012, p. 80). Neben diesen Netzwerken sind insbesondere auch jene innerhalb Österreichs relevant. Das soziale Netzwerk einer Person inkludiert unterschiedliche soziale Beziehungen, wodurch soziales Kapital generiert wird und Ressourcen zur Verfügung stehen (z.B. Unterstützung, Informationen), die hilfreich sein können (DEINDL 2005; LIN 2001). Nach HAUG ist soziales Kapital in Anbetracht von Migration insbesondere für folgende Aspekte relevant: „Zum einen kann soziales Kapital Migrationsentscheidungen beeinflussen und zur Entstehung von Kettenmigration führen. Zum Zweiten hängt mit dem sozialen Kapital die soziale Einbettung und die Integration am Zielort zusammen“ (HAUG 2007, p. 90). Netzwerke bieten jedoch nicht nur Hilfe an, sondern sind gekoppelt an den eigenen Input in das Netzwerk, der sich z.B. anhand von Gegenleistungen oder normkonformem Verhalten zeigt. Sie verfügen jedoch nicht nur über positive Ressourcen, sondern können z.B. auch sozialen Druck ausüben (ebd. 2007). Es stellt sich daher die Frage, inwiefern unterschiedliche Dynamiken der Netzwerkeinbindung und Veränderungen der sozialen Beziehungen einen Einfluss auf die Lebensgestaltung, Integrationsbemühungen und das allgemeine Wohlbefinden haben. 


\subsubsection{Soziale Beziehungen zu Personen aus der österreichischen Mehrheits- gesellschaft (privat und institutionell)}

Kontakte zur österreichischen Mehrheitsgesellschaft werden als essentiell für vielfältige Aspekte des Ankommens und Zurechtfindens in Österreich betrachtet. Einen Zugang zu dieser Personengruppe zu finden gilt für die Geflüchteten aufgrund einer erwarteten leichteren Integration in die österreichische Gesellschaft als besonders erstrebenswert, wenngleich dies teilweise mit Schwierigkeiten verbunden ist. Analysiert man dies anhand der Aufenthaltsdauer und des Asylstatus, so werden Schwierigkeiten der Kontaktaufnahme vor allem auf die Wohnsituation in Grundversorgungsquartieren zurückgeführt. Die meisten Kontakte haben Asylwerber/innen dort mit Bewohner/inne/n desselben Quartiers oder auch mit Sozialarbeiter/inne/n und Freiwilligen: ,Im Heim war es schwierig [Kontakt zu Österreicher/Inne/n zu finden], weil dort nur Flüchtlinge gewohnt haben und der Spracherwerb war auch schwierig. " (I28, weiblich, *1977, seit 2013 in Ö.)

Auch in den Kursen, die im Laufe des Integrationsprozesses eingefordert werden (wie z.B. Sprach- oder Wertekurse), ist der Aufbau sozialer Beziehungen hauptsächlich auf Personen aus der eigenen Community oder jene mit Fluchthintergrund aus anderen Herkunftsländern beschränkt.

„I: Also war es leicht für dich dann Leute kennenzulernen? B: Am Anfang nicht. War schwierig, weil ich war in einer Klasse und ich hab ' nur Persisch gesprochen und alle anderen konnten so ein bisschen Deutsch. Und alle anderen Kinder können ganz gut Deutsch und ich war nur allein. Aber es wird schon besser, weil es ist ein anderes Mädchen gekommen und sie spricht auch Persisch und wir waren zusammen - Freundschaft und so etwas - und es wird schon besser glaub " ich. I: Und hast du auch viele Österreicher kennengelernt? B: Österreicher nein, weil in meiner Schule und meiner Klasse gibt es überhaupt kein Österreicher. “ (I44, weiblich, seit 2015 in Ö.)

Kontaktpersonen aus der österreichischen Mehrheitsgesellschaft sind überwiegend Buddys, also Freiwillige aus der Zivilgesellschaft, die einzelne Geflüchtete beim Ankommen unterstützen, Mentor/inn/en oder Kursleiter/innen. Erst durch die Eingliederung in reguläre Wohnsituationen, Schulen, Arbeitsstellen oder z.B. die Teilnahme an Initiativen von Freiwilligen wird der Aufbau sozialer Beziehungen mit Personen der Mehrheitsgesellschaft einfacher.

„Also in Kärnten und Leoben [im Asylheim] war nicht erlaubt in die freie Gesellschaft rauszugehen. Wir waren immer in einem Heim geschützt sozusagen, wir waren immer in einem Heim. Wir konnten nicht rausgehen. Ja schon, wenn wir Termine hatten. Zum Arzt konnten wir gehen, ansonsten im Bazar war ich nicht. Ich kann auch nicht sagen, ob wir durften, weil ich selber nicht dorthin gegangen bin. Aber hier als ich in Eferding war, habe ich Kontakte knüpfen können. In der Schule oder mit Freiwilligen, die zu uns gekommen sind. Die Tanzschule habe ich besucht und ich fahre ab und zu nach Wien, Konferenzen zu halten, und dort habe 
ich viele Leute gefunden und viel mehr internationale Kontakte geknüpft. “ (I18, weiblich, seit 2016 in Ö.)

„I: Hast du Kontakte zu Österreicherinnen? B: Also früher nicht, aber jetzt, weil ich arbeite, habe ich Kontakt zu meinen Kollegen. ... Mit denen gehen wir schon manchmal am Wochenende spazieren oder in einen Café-Shop. Einfach, um zu reden und zu sitzen und so was. Und es ist so, wenn du keinen Kontakt zu den Leuten hast, weißt du nicht, wie die Leute sind. " (I1, männlich, seit 2013 in Ö.)

Doch auch wenn im alltäglichen Leben objektiv betrachtet einfache Kontaktmöglichkeiten gegeben sind, bestehen weiterhin Hürden des Beziehungsaufbaus. Diskriminierungserfahrungen sowie Vorurteile von Seiten der Mehrheitsgesellschaft gegenüber Geflüchteten im Allgemeinen und gegenüber Afghan/inn/en und Muslim/inn/en im Besonderen erschweren den Aufbau sozialer Beziehungen mit Österreicher/inne/n:

„Es ist auch manchmal schwierig in Österreich, die Leute sind eher verschlossen. “ (I2, männlich, *1998, seit 2015 in Ö.)

„Ich fühle mich in die Gesellschaft integriert und ich fühle mich auch wohl. Aber wenn manche Leute manche Sachen sagen, dann denke ich: Ich bemühe mich und trotzdem gehöre ich nicht dazu. Das tut schon weh. " (I32, weiblich, *1975, seit 1995 in Ö.)

Meist werden Diskriminierungserfahrungen in Österreich von afghanischen Geflüchteten, welche einen Teil ihres Lebens im Iran verbracht haben, mit den dortigen Erfahrungen in Verbindung gebracht und damit relativiert.

„Ja, ich habe auch Lebenserfahrung von Iran. Obwohl Sie meine Sprache sprachen, wurde ich als ein Afghane verachtet und als minderwertig angesehen. Hier ist es anders. Die Leute wissen, dass ich ein Ausländer bin, aber trotzdem respektieren sie mich und nehmen mich als einen Menschen wahr. Wenn man ihre Sprache spricht, dann stellt man fest, dass sie kommunikativ sind und sich sehr gerne mit dir unterhalten und dir helfen möchten. " (I50, männlich, seit 2015 in Ö.)

Neben Diskriminierungs- und Ausgrenzungserfahrungen werden auch sprachlich und kulturell bedingte Verständigungsprobleme als anfängliche Hürden der Kontaktaufnahme genannt. Die Überwindung derselben benötigt Zeit und setzt ein Erlernen der deutschen Sprache sowie einen kulturellen Austausch zwischen Herkunfts- und Aufnahmegesellschaft voraus.

„I: Haben Sie das Gefühl, dass Sie ein Teil dieser Gesellschaft sind? Fühlen Sie sich wohl in dieser Gesellschaft? Wieso? B: Noch nicht zur Gänze, aber ich gebe mein Bestes, dass ich die Sprache und Kultur kennenlerne, aber noch bin ich nicht zur Gänze ein Teil. I: Wieso? B: Weil Dinge zwischen zwei Kulturen unterschiedlich sind. Und es ist schwierig, die neue Kultur so schnell aufzunehmen. Es braucht seine Zeit. " (I54, männlich, seit 2015 in Ö.) 
Personen aus der österreichischen Mehrheitsgesellschaft als einen Bestandteil des eigenen sozialen Netzwerks bezeichnen zu können, kann für Geflüchtete einige positive Aspekte mit sich bringen. Besonders erwähnenswert sind hierbei: Hilfe bei Amtswegen/ Bürokratie, Erlernen der deutschen Sprache, Vermittlung von Jobs/Wohnungen, Familienersatz, Freundschaften als Beitrag zum Wohlbefinden sowie Kulturaustausch, wobei einander diese Bereiche meist oder oft überlappen.

\subsubsection{Hilfe bei der strukturellen Integration (Amstswege Behörden, Arbeitsver- mittlung Wohnen)}

Vom Asylantrag über die Anmeldung zu Kursen, Arztbesuchen bis zu AMS-Terminen und Mietvertragsunterzeichnungen, die bürokratischen Anforderungen in Österreich stellen für geflüchtete Afghan/inn/en nicht nur sprachlich, sondern auch aufgrund einer fehlenden Sozialisation in diesen Strukturen eine anfängliche Überforderung dar. Personen aus der Mehrheitsgesellschaft repräsentieren als Freund/e/innen, Buddies, Sozialarbeiter/innen oder Mentor/inn/en eine wesentliche Unterstützung. Personen, die in der Anfangszeit in Österreich über NGOs, wie das Rote Kreuz oder die Caritas, kennengelernt wurden, werden oft zu wichtigen und langfristigen Bezugspersonen und Unterstützer/inne/n im Alltag.

„,Weil meine gute Erfahrung war, als ich mit dieser Österreicherin Freundin geworden bin. Ich habe sie schon beim Roten Kreuz gefunden ... Die Frau heißt $X X$, und sie war sehr nett und sie ist einmal pro Woche zu uns gekommen und hat beim Papier viel geholfen und unsere Papiere vom Sozialamt, AMS oder so beantwortet. Und sie hat mir viel geholfen bei der Sprache und beim Lernen und meinen Kindern auch viel geholfen. Wir sind seit fünf oder sechs Jahren Freundinnen und ich liebe diese Frau und sie mich auch. " (I19, weiblich, *1973, seit 2011 in Ö.)

Neben der Unterstützung bei Amtswegen und Behördengängen gilt auch die Vermittlung von Arbeitsplätzen und Wohnungen als wichtiger Unterstützungseffekt durch Personen der österreichischen Mehrheitsgesellschaft, welche die Geflüchteten damit an ihrem eigenen sozialen Netzwerk teilhaben lassen.

\subsubsection{Erlernen der deutschen Sprache}

Neben fehlenden Möglichkeiten während der Unterbringung in einem Gemeinschaftsquartier soziale Beziehungen zu Personen der österreichischen Mehrheitsgesellschaft aufzubauen, wird auch die eingeschränkte Möglichkeit Deutsch, abseits von Sprachkursen und Eigeninitiativen (z.B. durch Youtube-Videos), im Alltag zu sprechen und zu üben als integrationshemmend bewertet (,, Und in der Zeit, in der wir warten mussten, habe ich eine Seite im Internet gefunden und habe dort Deutsch gelernt "; I17, männlich, *1997, seit 2013 in Ö.). Im Gegensatz zu einer Unterbringung bei österreichischen Familien oder in Privatwohnungen mit Nachbar/inne/n aus der Mehrheitsgesellschaft werden in Grundversorgungsquartieren hauptsächlich bereits erlernte Sprachen oder die Erstsprachen (z.B. Englisch, Farsi/Dari/Paschtu) zur Kommunikation einge- 
setzt. Das Erlernen des Deutschen wird von den Geflüchteten als einer der wesentlichsten Einflussfaktoren für die Eingliederung in die Gesellschaft angesehen:

„I: Wie fühlen Sie sich in Österreich? Fühlen Sie sich den Österreichern zugehörig?

B: Ja, ich fühle mich den Österreichern zugehörig, aber noch nicht ganz. Erst nachdem ich die Sprache perfekt gelernt habe und nachdem ich eine Arbeit habe, werde ich mich den Österreichern angepasst fühlen. " (I57, weiblich, seit 2015 in Ö.)

Daher wird es auch als hilfreich - wenn auch herausfordernd - empfunden, sowohl in der Nachbarschaft als auch bei Freizeitaktivitäten die Möglichkeit zu haben, Deutsch als Alltagssprache zu benutzen.

„,Und wenn man österreichische Freunde hat, ist dies auch sicherlich eine große Hilfe in Bezug auf Arbeit und vor allem beim Lernen. Neben dem Deutschkurs bin ich noch in einer anderen Gruppe, mit der wir auch Ausflüge machen. Die Gruppe hilft uns sehr. Sogar als ich mein Interview hatte, sind sie mit uns gegangen. Sie haben uns auch, was das Lernen angeht, sehr unterstützt. Immer wenn ich Probleme dabei hatte oder etwas nicht wusste, haben Sie mir geholfen." (I65, männlich, seit 2015 in Ö.)

Neben der freundschaftlichen Unterstützung in schwierigen Situationen ist die Hilfe beim Spracherwerb ein wichtiger Aspekt der Sozialkontakte zwischen afghanischen Geflüchteten und Österreicher/inne/n. Die deutsche Sprache zu erlernen wird meist als wichtigster Faktor für das Ankommen in Österreich beschrieben.

„I: War es wichtig für Sie, die deutsche Sprache zu lernen? B: Zu 100\%, weil die Sprache der Schlüssel für den Einstieg in die Gesellschaft ist, und wenn man diesen Schlüssel nicht hat, egal wie gebildet man sein mag, kann man sich nicht ausdrücken und mit den anderen kommunizieren. Für mich war es schwierig, die Sprache zu lernen am Anfang, aber ich habe mein Bestes gegeben, durch einen guten Freund die Sprache so rasch wie möglich zu lernen. Ich habe eine Frau über die Universität Wien kennengelernt, die aus Luxemburg kommt und die Dari lernen möchte. Ich helfe ihr und sie hilft mir. Und ich kann die deutsche Sprache viel besser als einige Jugendliche, die mit mir im Jugendcollege sind, weil sie keinen Kontakt zu den Österreicher/inne/n haben, deswegen fällt es ihnen schwerer. " (I54, männlich, seit 2015 in Ö.)

\subsubsection{Familienersatz und Freundschaften}

Sozialkontakte und Netzwerkeinbindungen können nicht rein aus zweckorientierter Perspektive betrachtet werden. Freundschaftliche Unterstützung und familiärer Beistand in einer neuen Lebenssituation sind ein wesentlicher Beitrag zum Wohlbefinden und damit ein Grundbaustein für weitere Integrationsbestrebungen. Neben Kontakten in der eigenen Community werden enge Beziehungen zu Personen der österreichischen Mehrheitsgesellschaft als wünschenswert eingestuft bzw. das Fehlen derselben negativ bewertet. 
„, Wien ist für mich eine sehr ruhige Stadt und ich fühle mich hier wohl. Es kann sein, dass ich mich manchmal alleine fühle. Es liegt daran, dass ich hier nicht viele Bekannte oder Freunde habe, aber ich habe hier eine österreichische Familie, die ich als meine Familie bezeichne. Der größte Wert, der mir entgegengebracht wird in dieser Stadt ist, dass man mich als Mensch wahrnimmt und mich respektiert. Und wenn du Ziele hast und dafür arbeitest, erreichst du sie auch. " (I48, männlich, seit 2014 in Ö.)

Ein wichtiger Effekt von freundschaftlichen Beziehungen mit Österreicher/inne/n und dem intensiven Kontakt mit dieser Personengruppe bildet der Austausch zwischen unterschiedlichen kulturellen Kontexten.

viele Freundinnen sind von hier. Ja, aber weniger afghanische. ... Es war ein gutes Gefühl für mich, weil ich konnte neue Kultur sehen. Zum Beispiel neue Kultur verstehen, neue Religion. Obwohl ich hatte schon ein bisschen Information darüber. Aber es ist anders, wenn man erzählt. Ein Beispiel: Die Freundinnen sitzen oder erzählen von zwei verschiedenen Ländern. Das habe ich gut gefunden. Und jetzt auch wir sind alle in Kontakt miteinander. Zu manchen Festen laden wir unsere Kolleginnen oder unsere Freundinnen ein oder die werden uns einladen. " (I24, weiblich, seit 2015 in Ö.)

\subsubsection{Soziale Beziehungen zu Angehörigen der eigenen ethnischen bzw. religiösen Community}

Die Einbindung in Netzwerke der eigenen ethnischen und/oder religiösen Community spielt eine wesentliche Rolle für das Ankommen in der österreichischen Gesellschaft, aber auch für die Aufrechterhaltung der Verbindungen zum Herkunftsland.

„,Das ist eigentlich für die Integration - denke ich -, dass es gut ist, wenn man mit den Einheimischen viel Kontakt hat und viel zu tun hat als nur in der Gesellschaft in der eigenen Community ... Ich persönlich meine, es ist beides wichtig, sowohl der Kontakt zu der ... Aufnahmegesellschaft oder Österreichern, aber auch Kontakt zu der eigenen Community, weil hier braucht man auch ein bisschen soziale Kontakte, ... Kontakte aus der Heimat. Auch für die Anerkennung, weil dann weiß man, ok, ich bin nicht hier alleine, sondern es gibt auch andere, die was gemacht haben, aber auch als Rollenbild, ,role models', also zum Beispiel ... Bin neuer Flüchtling, komme nach Wien, dann plötzlich treffe ich einen Afghanen, der bei diesem Amt oder dort als Arzt arbeitet ... Dann plötzlich werde ich auch motiviert und sage: ,Ok, was hast du gemacht? Und wie hast du es geschafft?" (E6)

Hinsichtlich sozialer Beziehungen zu Personen der eigenen ethnischen Community können neben ähnlichen Effekten wie beim Netzwerk mit Österreicher/inne/n (Vermittlung von Wohnungen/Jobs, familiärer Beistand etc.) weitere positive sowie 
negative Auswirkungen festgestellt werden. Die Aspekte der sozialen Unterstützung, des Informationsaustausches, der Teilhabe an wichtigen Lebensereignissen sowie des kulturellen Ankers (u.a. durch afghanische Kultur- oder Moscheevereine) spielen eine wesentliche Rolle. Des Weiteren kann eine teils starke Distanzierung von der eigenen ethnischen Community beobachtet werden.

\subsubsection{Soziale Unterstützung - Informationsaustausch}

Ähnlich wie bei der Einbindung in österreichische Familien (z.B. Patenschaften) werden Sozialkontakte mit Personen der eigenen ethnischen Community oft mit einem Familienersatz in Verbindung gebracht. Viele leben fern ihrer Familienangehörigen und engen Freund/inn/e/n. Diese befinden sich teils noch im Herkunfts- oder in einem Transitland oder haben nicht in Österreich einen Asylantrag gestellt, womit eine weltweite Vernetzung mit Personen der eigenen ethnischen Community zu beobachten ist.

„Als mein Vater weg war, sind dann die großen Geschwister auch nach Deutschland, nach Niederlande, nach Amerika einfach so weg von zuhause. Bis mein Bruder mich nach Österreich geholt hat. " (I22, männlich, seit 1986 in Ö.)

Aufgrund der anfänglichen sprachlichen Schwierigkeiten wird der Kontakt zu Personen der afghanischen Community besonders in der Anfangszeit in Österreich als hilfreich für das persönliche Wohlbefinden eingeschätzt.

„I: Und war es dann irgendwie auch wichtig für euch, dass andere Afghanen dort [in Linz] leben? B: Na, anfangs war's halt so, es gab nur uns und zwei weitere Familien. Jahrelang war das so. Selbst die ersten 10 Jahre als wir da gelebt haben, kannten wir gar keine Afghanen, und ich muss schon sagen, dass man sich schon sehr oft einsam gefühlt hat und man hat sich auch gefreut, wenn man andere Afghanen kennengelernt hat. Zum Beispiel meine Eltern hätten sich definitiv gewünscht, dass mehr Afghanen hier leben, in unserer Stadt. Die ersten Jahre waren definitiv sehr einsam. “ ( I45, weiblich, seit 1992 in Ö.)

Unterschiede hinsichtlich des Bedürfnisses nach Kontakten zu Personen mit afghanischem Migrationshintergrund können nicht nur an der Dauer des Aufenthalts in Österreich, sondern unter anderem anhand von Generationsunterschieden festgemacht werden. Vor allem ältere Personen, die, anders als jüngere, nach ihrer Ankunft in Österreich vermehrt kommunikative Schwierigkeiten mit Deutsch sprechenden Personen äußern, schätzen den sozialen Austausch mit der eigenen ethnischen Community und ein Verbundenheitsgefühl zum Herkunftsland. Es wird auch deutlich, dass Integrationsmaßnahmen, wie etwa Deutsch- und Wertekurse, an das Alter sowie den Bildungshintergrund der Teilnehmer/innen angepasst werden müssen. In Anbetracht psychisch belastender Situationen und Probleme wird soziale Unterstützung innerhalb der eigenen ethnischen Community vor allem auf Basis eines geteilten Erfahrungsschatzes als Familienersatz geleistet. 
„I: Aber es ist für dich wichtig, dass du da Kontakt zur afghanischen Community hast? ... B: ... Halt, wenn ich was machen will, dann mein Gehirn funktioniert nicht, darüber nachzudenken. Brauche ich Hilfe von ihm, von einem anderen Freund von mir. Dann helfen sie mir. Und das ist natürlich sehr gut. I: Also sie helfen dir auch Entscheidungen zu treffen, wenn du nicht weiter weißt oder wenn du Probleme hast? B: Ja, weil wir da keine Familie haben. " (I16, männlich, seit 2012 in Ö.)

Des Weiteren kann eine Unterstützung in der Erstsprache bei anfänglich schlechten Deutschkenntnissen das Verständnis für die Strukturen der österreichischen Gesellschaft und eine Integration in den Bildungssektor und den Arbeitsmarkt erleichtern (vgl. Wohnund Arbeitsmarktintegration). Erfahrungen von bereits länger in Österreich lebenden Afghan/inn/en können auf diese Weise besser mitgeteilt werden, als es ein Angehöriger der Mehrheitsgesellschaft vermag.

„B: ... Die Beste war die Freundin von diesem afghanischen Studentenverein. Die haben mir wirklich sehr viel geholfen ... Uni, Anmelden, Wohnung finden in Wien. Ich habe sogar mit einer von denen in einer WG gelebt am Anfang in Wien. Und ich habe bis drei Monate nicht gezahlt, bis ich wieder mein Geld bekomme vom Staat. Die haben mir wirklich sehr viel geholfen, und auch mit Integration ..., weil von denen kann ich mehr lernen am Anfang als von den Österreichern. Weil es gibt viele Gemeinschaften [Gemeinsamkeiten] und auch Sprache usw. Weil am Anfang, wenn mir jemand über ein bekannteres Restaurant in Wien auf Deutsch erzählt, das werde ich gar nicht verstehen. Aber auf Farsi-ok-das ist ein gutes Restaurant, dann geht es in mein Unterbewusstsein. Und ich werde mir sagen: ,Das muss ich besuchen'. Das ist ein Schritt von Integration. “ (I3, männlich, seit 2014 in Ö.)

Der Austausch von Informationen kann - im Gegensatz zu den weitgehend formalisierten Informationsflüssen in österreichischen Behörden oder NGOs - innerhalb der Community auf informelle Weise geschehen und mit Freizeitaktivitäten verbunden werden.

„Kulturvereine bei Afghanen ist nicht so richtig ausgeprägt. Es gibt nicht so viele und die sind nicht so richtig aktiv. Aber die afghanischen Communities, also dieses Netzwerk, das sie haben, funktioniert sehr gut. Also dieser Informationstausch, unterstützende Sportvereine, welche die haben, also die funktionieren sehr gut." (E4)

Häufig werden in diesem Zusammenhang ähnliche Erfahrungen als Basis für ein gegenseitiges Verständnis erwähnt, wodurch von Anfang an Gefühle einer gewissen „Verbundenheit“ untereinander bestehen.

„, Weil bei ihm war es z.B. so, dass wir uns in der Unterstufe schon kennengelernt haben. Und da war es so, er war ein Afghane und ich war ein Afghane und da war es so: ,Hey, du bist Afghane, cool! Alles klar! Da haben wir uns halt viel verbundener gefühlt und so. " (I5, männlich, seit 2006 in Ö.) 
Eine Unterstützung hinsichtlich des Aufbaus eines neuen Lebens in Österreich und bei Integrationsmaßnahmen ist jedoch nicht nur auf informeller Basis vorhanden, sondern auch in institutioneller Ausprägung, z.B. durch NGOs oder Vereine. Geflüchtete fungieren hier sowohl als Empfänger/innen als auch als aktiv Mitwirkende von Unterstützungsangeboten.

„I: Mit den afghanischen Leuten haben Sie auch Kontakt? B: Ja, nicht so viel, aber beim Deutschkurs und Nachbarin oder Nachbar, ja, wir haben Kontakt. Ich habe immer allen geholfen. Dolmetschen im Asylheim in Bad Kreuzen, ja, in Linz, immer. " (I26, männlich, seit 2013 in Ö.)

„B: Ja, es gibt halt einen afghanischen Verein hier. Und dort engagiere ich mich, dort bin ich auch aktiv und zwar, dass ich immer, ich helfe dort auch manchmal mit. ... Aber ich unterrichte halt immer die Flüchtlinge. Und das mach ich schon seit, ich glaub', seit letztem Jahr Mai oder April. Wir versuchen einfach die Flüchtlinge zu fördern, ihnen zu helfen, in jeglicher Hinsicht, ihnen zu helfen und so weiter, Alphabetisierungskurse und ja ... man merkt einfach, dass man sich damit gegenseitig unterstützt. Die veranstalten zum Beispiel auch Fußballspiele oder mehrere Feiern und man merkt, dass man irgendwie dann die Afghanen zusammenbringt. “ (I45, weiblich, seit 1992 in Ö.)

Die Integrationsunterstützung durch die eigene Community schließt auch die Möglichkeit mit ein, einen Wertekurs bei einem der afghanischen Vereine in Österreich zu absolvieren. Dadurch können Inhalte des Kurses aus der Perspektive der eigenen ethnischen/religiösen Community vermittelt werden.

\subsubsection{Distanzierung gegenüber Angehörigen der eigenen ethnischen bzw. religiösen Community}

Weitgehend simultan zur positiven Bewertung der Unterstützung und des „Haltgebens“ während der Phase des Ankommens in Österreich ist eine teils starke Distanzierung gegenüber Personen der eigenen ethnischen/religiösen Community zu beobachten. Diese Ambivalenz wird auf der Grundlage unterschiedlicher Motivationen begründet. Dabei ist eine Distanzierung basierend auf z.B. religiösen Unterschieden, dem sozialen Druck innerhalb der Community sowie konservativem versus modernem Denken zu erkennen. Häufig wird ein dem traditionell afghanischen Verhaltenskodex entsprechendes normkonformes Handeln eingefordert. Trotz der Distanzierung im Alltag besteht bei den Respondent/inn/en aber weiterhin ein Gefühl der Verpflichtung zur Teilnahme an Feierlichkeiten, wie z.B. Hochzeiten oder Trauerfeiern.

„Ich hab' eigentlich wenig Kontakt mit Afghanen. Ist mir egal, wenn ein Afghane gut ist, nicht viel so bla, bla, bla. ... Ja, ist kein Problem für mich. Manchmal machen Afghanen Probleme wegen allem. Wegen Kleidung, wegen wieso du so, so. Deswegen ich muss ein bisschen aufpassen. " (I42, weiblich, seit 2014 in Ö.)

Der Wunsch, sich seinen eigenen Vorstellungen gemäß zu verhalten und sich z.B. für die eigene Kleiderwahl oder die praktizierenden Freizeitaktivtäten gegenüber der 
Community nicht rechtfertigen zu müssen, führt in einigen Fällen zu einem Rückzug bzw. zu einer Reduzierung der Sozialkontakte in der ethnischen und religiösen Community.

„,Wenn du mit denen zusammen bist, dann musst du das machen, was die wollen. Wenn du was Anderes machst, dann wird über dich gesprochen, wird über dich geschimpft. Aber wenn du dich jetzt weg von denen hältst, dann hast du ein freies Leben. " (I7, weiblich, seit 2001 in Ö.)

Die Erwartung sich normenkonform zu verhalten wird auch mit der religiösen Praxis in Verbindung gebracht. Moscheen werden zwar für Feierlichkeiten genutzt, eine starke Einbindung wird jedoch vielfach abgelehnt und oftmals in Hinsicht auf einen Schutz der jungen Generation vor radikalen Denkweisen vermieden.

„I: Und besucht ihr oft die Moschee in Österreich? B2: Nein, ich gehe nicht, weil dort sind sie ein bisschen streng mit Frauen. Sie sagen: ,Warum hast du Hose getragen? Du musst einen Rock haben. Langen Rock haben. Warum hast du das getragen? Warum hast du so gemacht? Warum hast du Nagellack gehabt? ... Deswegen ich will nicht dorthin gehen. Ich mache meine Gebete zu Hause, wenn ich Zeit habe. ... ich habe keinen Kontakt mit diesen Leuten, weil sie sprechen über Frauen und deshalb mache ich es nicht. Ich habe nur wenige Freunde. Alle sind open minded. ... B1: Weil ich gehe nicht, weil das ist gefährlich für unsere Kinder. Wenn sie einmal zu dieser Religion gehen, dann ist das ein dem traditionell afghanischen Verhaltenskodex entsprechendes normkonformes Handeln eingefordert ... Wenn sie zur Moschee gehen, sagt der Mullah: ,Machst du so, machst du so '. Dann nützen sie unsere Kinder aus. Wir lassen unsere Kinder nicht in die Moschee, nie. “ (I19, weiblich, seit 2011 in Ö.; I20, weiblich, seit 2013 in Ö.)

Daneben muss auch die Wahrnehmung afghanischer Geflüchteter durch die Mehrheitsgesellschaft in die Analyse der Distanzierungstendenzen miteinbezogen werden. Das negative Bild der Afghan/inn/en im öffentlichen Diskurs führt zu einer vordergründigen Distanzierung gegenüber der afghanischen Community. Man möchte mit den Vorurteilen und negativen Eigenschaften, die afghanischen Geflüchteten zugeschrieben werden, nicht in Verbindung gebracht werden.

„Aber im Ort, wo ich lebe, mag ich, dass keine Afghanen leben. Ich meine, es sollten jene leben, die gut sind. Weil, egal ob Österreicher oder Afghane sind sowohl gut oder schlecht. Und in meinem Wohnort ist es mir wichtig, was die Österreicher/innen darüber denken und wenn es positiv ist, würde ich mit ihnen wohnen, und wenn nicht, dann würde ich mich fernhalten. " (I56, männlich, seit 2015 in Ö.)

Eine Abwendung von der Community wird außerdem mit einer Distanzierung von ethnischen Konflikten begründet.

„Ich muss ehrlich gestehen, traurigerweise ist es zum größten Teil immer noch so .... ,Ah du bist Hazara!' oder ,Ah, du bist Tadschikin!' - was ich persönlich nicht mag. Weil wir sind jetzt in Europa, mittlerweile sollten wir schon aufhören damit! 
Weil ... ich hab' noch nie von Österreichern gehört: ,Ah, du kommst aus Tirol!', ,Ah, du kommst aus der Steiermark! - weißt du, was ich meine? ... Aber man kann ihnen nicht eine Gehirnwäsche geben und sagen: ,Menschen sind alle gleich!" Vielleicht solltet ihr das, was ihr dort gemacht habt, nicht mitnehmen und diesen Hass wieder hier neu beginnen! “ (I10, weiblich, seit 2006 in Ö.)

Es gibt auch jene, die aufgrund angenommener Differenzen und ethnischer Konflikte ausschließlich mit ihrer eigenen Volksgruppe Sozialkontakte pflegen.

„Es gibt schon in Afghanistan sehr viele Probleme immer mit diesen Leuten ... Aber ich kann nur gut mit Hazara-Leuten. Ich habe Kontakt mit anderen afghanischen Leuten, aber es gefällt mir nicht mehr. ... Die sind andere Gruppe von Leute, ganz anders. Kann ich nicht auf Deutsch sagen. Aber es gibt Hazara-Leute, wenn du etwas brauchst, gehst du zu [ihnen] [unverständlich]. " (I25, männlich, seit 2014 in Ö.)

Eine Distanzierung gegenüber der eigenen ethnischen Community kann also aus verschiedenen Gründen und in unterschiedlicher Ausprägung vorhanden sein.

\subsubsection{Wichtige Lebensereignisse}

Die Teilnahme an Feiern zu wichtigen Lebensereignissen wie Hochzeiten oder Begräbnissen kann als wichtiger Aspekt des Zusammenhalts betrachtet werden. Sich auf die Unterstützung der eigenen Community verlassen zu können, führt zu sozialer Absicherung und einem starken Gemeinschaftsgefühl.

„, Weil bei gewissen Sachen ist der Zusammenhalt auch groß, und man will auch dazu gehören irgendwie. Was z.B. Trauer betrifft, was so Hochzeiten betrifft, wenn es Schwierigkeiten, Lebensereignisse betrifft, dann sind sie da ... Nicht die Vereine, die Community. Aber die sind automatisch die Vereine. Und diese Vereine ermöglichen es der Community zusammenzuhalten. “ (I4, männlich, seit 2001 in Ö.)

Neben der Unterstützung seitens der Community in Österreich sind auch die transnationalen Vernetzungen zu den Angehörigen im Herkunftsland bzw. in Transitländern und anderen Aufnahmeländern relevant. Soziale Kontakte innerhalb der Community sind nicht geographisch auf Österreich abgegrenzt $\mathrm{zu}$ betrachten, sondern müssen in einem größeren räumlichen Kontext - oft international - eingebettet verstanden werden. Die Teilhabe an wichtigen Lebensereignissen bildet einen wesentlichen Faktor des Netzwerks. Der Aspekt der sozialen Verpflichtung innerhalb der Community wird hierbei deutlich.

„Aber wir haben schon Kontakt mit vielen Afghanen und wenn z.B. ein Fest oder eine Hochzeit oder eine Trauerzeremonie oder so ist, gehen meine Eltern z.B. schon oft hin, weil das gehört sich irgendwie sozusagen. Das gehört sich, dass die Älteren sich dann besuchen oder zu den Trauerfeiern oder zu den Hochzeiten und so gehen. " (I5, männlich, seit 2006 in Ö.) 


\subsubsection{Rolle der afghanischen Kultur- und Moscheevereine}

Afghanische Vereine und Moscheevereine spielen eine wesentliche Rolle im Rahmen der Ausübung bestimmter Feierlichkeiten, wie Trauerfeiern oder religiöser Feiertage, spielen. Zugleich tragen Kulturvereine zur weiteren sozialen Vernetzung innerhalb der Community bei, unterstützen bei Integrationsbestrebungen wie dem Spracherwerb, stärken die Community durch Veranstaltungen und tragen zum Kulturaustausch bei. $\mathrm{Zu}$ unterscheiden sind hier Kultur- und Moscheevereinen, wobei letztere sich hauptsächlich auf die Ausübung der Religion fokussieren.

„,... eigentlich alle Vereine, die ich kenne, die engagieren sich immer wegen Kultursachen usw. und nicht wegen der Religion. Es gibt schon vielleicht einige, die auch in der Religion tätig sind, weil sie ihr Budget von irgendwo anders bekommen ... Weiß ich nicht, aber wir dürfen auf keinen Fall einen Kulturverein mit einem ... Moscheeverein verwechseln ... " (I3, männlich, seit 2014 in Ö.)

Moscheevereine bieten einen räumlichen Rahmen für die Ausübung der religiösen Praxis sowie einen Ort des Zusammenkommens und des gemeinsamen Zelebrierens religiöser Feiertage und Trauerfeiern, wodurch ein gemeinschaftsstärkender Charakter hinzugewonnen werden kann.

„Die Moscheen spielen eine Rolle, dass die Leute dorthin gehen. Ja, vor allem ... die Totengebete werden dort abgehalten. Die Leute beten, die in der Moschee beten wollen, ältere Personen, gehen hin. So weit ist nichts gegen die Moscheen zu sagen. Das ist immer eine Institution - Moschee oder Verein - das hat eine bestimmte Rolle in der Gesellschaft, für den Zusammenhalt der Gesellschaft. “ (E3)

Moscheen als Orte des Zusammenhalts und der Solidarität mit Angehörigen der eigenen ethnischen und religiösen Community werden auch von nichtpraktizierenden Personen als solche genutzt, eine Abgrenzung zur religiösen Praxis wird jedoch vielfach betont.

„I: Und Moscheen und so, besuchen Sie eh nicht so oft oder? B: Zum Beten nein. Aber falls ich zu einer Trauerfeier eingeladen sein sollte, dann schon. ... Die Mullahs, die dort predigen, kennen mich alle und wissen auch wofür ich stehe und wie meine Haltung zur Religion ist. ... Nein, mit denen habe ich überhaupt keinen Kontakt. Und egal welche gute Tat ich vollbringe, ich tue dies im Namen der Menschlichkeit und nicht der Religion willen. Wenn ich sehe, dass jemand meine Hilfe braucht, dann helfe ich dieser Person im Namen der Menschlichkeit. Und ich tue dies ohne eine Gegenleistung zu verlangen. Ich bin auch seit 25 Jahren Mitglied in einem afghanischen Kulturverein. Seit 25 Jahren helfe ich afghanischen Flüchtlingen. " (I64, männlich, seit 1983 in Ö.)

Die Abgrenzung zum Kulturverein dessen Fokus nicht auf der Religion liegt, spielt eine wesentliche Rolle beim Engagement in den Vereinen. Anhand der Gründungsintentionen und der Organisationsstruktur werden die Ziele und Aufgaben der Vereine deutlich. 
„Hauptziel von dem war, dass die Afghanen, die Flüchtlinge, da eine Art Zentrum haben und über dieses hinaus durch Kontakt mit Österreichern gewissermaßen eine Art Integration stattfindet. Aber leider wurde dieser Verein gewissermaßen immer politisiert und dann habe ich nicht mitgemacht. Und dann hat man den Verein aufgelöst. Aber irgendwie durch Kontakt mit so vielen anderen Vereinen versucht man, dass irgendwie eine Art, ein Netzwerk da ist, dass die Leute sich gegenseitig verstehen bzw. etwas mitteilen, also Feste vereinbaren und Einiges gemeinsam tun. ... die Vereine, wenn sie wirklich für die Integration arbeiten, die sind wunderbar. Die sind gute Institutionen, die sollte man nur unterstützen. “ (I37, männlich, seit 1988 in Ö.)

Anlass für die Gründung der Kulturvereine bildete die Vermittlung eines Bilds der afghanischen Kultur und Gesellschaft, das sich von dem stark konservativ orientierten Leitbild des Talibanregimes abgrenzt und die kulturelle Vielfalt Afghanistans in Österreich sichtbar macht. Weiterhin standen Organisation und Angebot von diversen Freizeitveranstaltungen (wie z.B. Sportaktivitäten oder Musikveranstaltungen) und Beschäftigungskursen, die sowohl einen strukturellen als auch sozialen Charakter aufweisen (u.a. Alphabetisierungskurse für Frauen) im Fokus. Ein Dialog innerhalb der heterogenen afghanischen Community sowie ein interaktives Zusammenkommen mit der Mehrheitsgesellschaft sollen einen Kulturaustausch initiieren und soziale Kontakte stärken.

„Darum sage ich: Nein, keine Politik, sondern Kultur zu präsentieren. Was kann man den Österreichern von unserer Kultur präsentieren, weil wenig Österreicher haben Informationen über uns gehabt und was kann man hier machen, was kann man von der österreichischen Kultur nehmen? ... und auch etwas Besseres von uns kann man geben. "(E11)

Zusätzlich schaffen die Vereine auch einen Raum, der es ermöglicht, eine Verbindung zur Kultur des Herkunftslandes zu pflegen sowie einander soziale Unterstützung anzubieten.

„Im Kulturverein war es z.B. unsere Aufgabe, unsere Muttersprache nicht zu vergessen. ... Wir sollten nicht das Jahresfest, das wir in unserem Heimatland gemacht haben, ... vergessen. Weil irgendwie wir brauchen das ja? Wir haben das als Gewohnheit. “ (I8, männlich, seit 2012 in Ö.)

Zusätzlich bieten die Kulturvereine neben den Angeboten des Österreichischen Integrationsfonds (ÖIF) die Möglichkeit Wertekurse oder Sprachkurse zu absolvieren, Informationen bereitzustellen oder bildungsbezogene Beratung durchzuführen.

„Das Ministerium, Innenministerium, Integrationsfonds, stellt den Vereinen Unterstützung zur Verfügung, dass sie selber, als afghanische Vereine, bestimmte Integrationsmaßnahmen haben, wie Wertekurse und so weiter, selber gestalten oder die Deutschsprachkurse gestalten. “(E3) 
Trotz der positiven Funktionen der Kulturvereine wird mit ihnen häufig die Problematik ethnischer Konflikte in Verbindung gebracht. Die Entwicklung mancher Kulturvereine und deren ethnische Fokussierung werden vor allem von Gründungsmitgliedern kritisch betrachtet.

„,Die Vereine wurden immer entlang der ethnischen Linien gegründet. Zum Beispiel dort findet man einen Verein von den Schiiten, einen Verein von den Paschtunen, einen Verein von den Tadschiken, und vor allem politische Organisationen. Und das ist etwas, was sehr bedauerlich ist. ... Es sollte eigentlich einen Verein für alle da sein. Dass alle, unabhängig von ihren politischen, religiösen oder kulturellen Identitäten zusammentreffen, dass sie gemeinsam etwas organisieren für mehr Zusammenhalt, für mehr Zusammenarbeit. Hilfe für die Neulinge, die herkommen. Und vor allem für die Erleichterung des Lebens, vor allem Hilfestellung. “ (I37, männlich, seit 1988 in Ö.)

An dieser Beschreibung ist eine Tendenz von vormals bestehenden Vereinen für die afghanische Community generell hin zu Vereinen für spezifische ethnische Gruppen zu erkennen. Das heißt aber auch, dass bei unterschiedlichen Gruppen divergierende Einstellungen zu einem gemeinschaftlichen Leben in Österreich vorhanden sind, wobei sich diese Problematik insbesondere in den Kulturvereinen manifestiert. Grundsätzlich lässt sich jedoch sagen, dass sich sowohl die Kultur- als auch die Moscheevereine durch eine Stärkung des Zusammenhalts innerhalb der Community auszeichnen, die Intention des Kulturaustausches betonen und die Ausübung von Feierlichkeiten ermöglichen.

\subsubsection{Soziale Beziehungen zu Angehörigen anderer österreichischer Minderhei- tengesellschaften}

Die Diversität der österreichischen Gesellschaft spiegelt sich zudem in den sozialen Netzwerken der Respondent/inn/en wider. Auch die Kontakte zu anderen Minderheiten sind mit den Bedingungen in den Asylwerber/innen-Unterkünften und den nachfolgenden Entwicklungen verwoben. Erste soziale Kontakte in Österreich bestehen daher meist zu Bewohner/inne/n des Asylquartiers sowie zu anderen Kursteilnehmer/inne/n.

„Nach meiner Ankunft habe ich ca. ein Jahr im 19. Bezirk gelebt. Dort waren meine farsisprechenden Freunde. Mit meinen Freunden aus dem Tanzkurs habe ich auch oft Kontakt. Ich gehe oft mit ihnen raus und auf Partys. Mit meinen Freunden und Bekannten, die ich noch aus dem Heim kenne, habe ich auch noch oft Kontakt. Mit denen fühl' ich mich am angenehmsten, da ich sie am längsten kenne. " (I62, männlich, seit 2015 in Ö.)

Die je nach Aufenthaltsdauer wachsenden Möglichkeiten der Eingliederung in reguläre Wohnsituationen, Arbeitsverhältnisse, Ausbildungsplätze und Freizeitaktivitäten lassen den zunehmenden Aufbau eines sozialen Netzwerks mit Personen aus unterschiedlichsten Herkunftsländern erkennen.

„Mein Freundeskreis, also ... eine Mischung aus allen. Meine beste Freundin ist aus Ägypten. Dann habe ich noch andere Freunde aus der Türkei, dann habe ich 
noch Freunde aus dem Balkan. Ich habe auch afghanische Freunde, ich habe auch österreichische Freunde, ja. “ (I12, weiblich, seit 2006 in Ö.)

Anzumerken ist hier, dass die Mehrheit der RespondentInnen über ein diverses und multikulturelles soziales Netzwerk verfügt. Dies wird sowohl mit der Schwierigkeit, soziale Kontakte zu autochthonen Österreicher/inne/n zu knüpfen, als auch mit der Annahme, dass Personen mit Migrationshintergrund ähnliche Lebenseinstellungen teilen, begründet.

„... Bei Ausländern ist es leichter, weil sie fast gleich denken wie wir. Aber mit Österreichern kannst du keinen guten Kontakt haben, bis du nicht dieselbe Arbeit, denselben Platz ... Du kannst eigentlich nicht so schnell Freund werden mit Österreichern. Es ist schwerer. Es dauert länger als mit Ausländern. " (I1, männlich, seit 2013 in Ö.)

Hinsichtlich der Zusammensetzung des individuellen sozialen Netzwerks und bezogen auf die unterschiedlichen Herkunftsländer, insbesondere was den engen Freundeskreis betrifft, werden unterschiedliche Begründungen angegeben. Als Basis werden z.B. ähnliche kulturelle Hintergründe, eine offenere Grundhaltung im Gegensatz zu Migrant/inn/en aus Afghanistan oder auch Ähnlichkeiten in der religiösen Praxis herangezogen.

„Ja, also jetzt habe ich wirklich selten Freundinnen, die aus meinem Land kommen. [lacht] Es sind meistens Freundinnen, die aus der Türkei kommen, aus der Slowakei kommen, die aus Ungarn kommen, die z.B. aus Kroatien oder Serbien oder Rumänien kommen. “ (I10, weiblich, seit 2006 in Ö.)

Allgemein wird die Wichtigkeit sozialer Kontakte jeglicher Herkunft hinsichtlich des individuellen Wohlbefindens in einem neuen Lebenskontext und der damit verbundenen Herausforderungen betont.

„Soziale Kontakte ... Ich glaube, alle sind wichtig. Weil wenn ich ein Problem habe, wenn ich nicht gut lernen kann ... würde ich es meinen Freundinnen oder aus meinem Heimatland oder aus anderen Ländern sagen. Deswegen sind alle für mich wichtig. Es ist nicht unterschiedlich. “(I2, männlich, seit 2015 in Ö.)

\subsubsection{Zugehörigkeit und Ausgrenzung}

Soziales Kapital, welches innerhalb sozialer Netzwerke akkumuliert werden kann, ist für die Einbettung in neue gesellschaftliche Verhältnisse und die Integration im Aufnahmeland ausschlaggebend (Haug 2007). Durch die Einbindung in soziale Netzwerke kann sich ein Gefühl der Zugehörigkeit entwickelt. Das Zugehörigkeitsgefühl der Geflüchteten ist stark geprägt von den Sprachkenntnissen, beruflicher Integration sowie der Intensität der sozialen Beziehungen zu Personen der österreichischen Mehrheitsgesellschaft.

„Ich habe das Gefühl ein Teil dieser Gesellschaft zu sein, aber noch nicht 100\%, vielleicht 50\%. Ich kann jetzt leicht mit den Menschen kommunizieren und fühle 
mich auch als Teil dieser Gesellschaft. Aber früher, als ich die Sprache noch nicht gut genug konnte, fühlte ich mich fremd. Ich meine, wenn ich irgendwohin wollte und Fragen hatte, konnte ich nicht leicht meine Antworten erhalten. Aber jetzt kann ich es. " (I48, männlich, seit 2014 in Ö.)

Infolge der Gewährung des Asylstatus, einer Verbesserung der Sprachkenntnisse oder der strukturellen Integration (z.B. Arbeitsmarkt, Bildungsmöglichkeiten) lässt sich auch ein steigendes Zugehörigkeitsgefühl erkennen.

„I: OK, fühlen sich heute in Österreich wohl und als Österreicher? B: Ja, ja, ich muss gestehen, ja. ... ich habe ein bisschen Glück gehabt sowohl bei der Arbeit als auch wo ich wohne. Ich habe eigentlich sehr liebe Leute, sehr angenehme Menschen kennengelernt. Mit denen verstehe ich mich sehr gut. ... z.B. unsere Nachbarinnen haben sich immer um unsere Kinder gekümmert. Unsere Nachbarin ist z.B. in die Schule gegangen, mit dem Deutschlehrer meiner Kinder z.B. unterhalten und auch Nachhilfe gegeben, oder versucht immer sie in verschiedenen Sportvereinen anzumelden. Oder mit ihnen gemeinsame Exkursionen zu organisieren. Oder auch gemeinsam mit eigenen Kindern Ausflüge zu machen. Also diese ganzen Erfahrungen sind sehr positiv. “ (I37, männlich, seit 1988 in Ö.)

Dennoch muss erwähnt werden, dass die Frage nach einem Gefühl der Zugehörigkeit zugleich mit der Erfahrung von Ausgrenzung einhergeht. Trotz einer Einbindung in soziale Netzwerke der österreichischen Mehrheitsgesellschaft und einer beruflichen Integration kann eine Zugehörigkeit niemals einseitig sein, sondern benötigt die Reaktion der Gruppen, denen man sich zugehörig oder nicht zugehörig fühlt.

„Ich fühle mich dazugehörig, aber, wenn ich an manche Situationen denke, fühlt man sich dann schon wieder als Ausländer. Ich habe letzte Woche ein Erlebnis in der Arbeit gehabt - wir sind seit zwölf Jahren zusammen, und dann sagt der eine zu mir: ,Ich werde für immer nach Polen ziehen, dort gibt es keine Muslime!' Und das tut dann schon weh. Oder so Ausländersprüche, wo man denkt: Wir arbeiten zusammen, ich habe mich integriert ... Also es gibt so gewisse Situationen, aber ansonsten ist alles ok. ... Ich fühle mich in die Gesellschaft integriert und ich fühle mich auch wohl. " (I32, weiblich, seit 1995 in Ö.)

Zugehörigkeit kann somit nicht auf einen bestimmten Status (Asylbescheid, Staatsbürgerschaft etc.), strukturelle Integrationserfolge oder die Einbindung in soziale Netzwerke reduziert werden. Sie ist abhängig von einem Konglomerat an Einflussfaktoren, welche wiederum stark mit einem respektvollen Umgang miteinander und gegenseitiger Anerkennung zusammenhängen.

\subsubsection{Zusammenfassung}

Wie sich gezeigt hat, ist die Einbindung in unterschiedliche Netzwerke - diese umfassen Angehörige der österreichischen Mehrheitsgesellschaft, der eigenen ethnischen/religiösen Community oder Personen aus anderen Minderheiten in Österreich - 
wesentlich für das Zurechtkommen in der Aufnahmegesellschaft. Aus jedem Netzwerk werden unterschiedliche Ressourcen gewonnen und in selbiges eigene Erfahrungen und Engagement eingebracht.

Unterstützung seitens aller drei beschriebenen Personengruppen bezüglich der strukturellen Integration (Arbeitsmarkt, Wohnen, Spracherwerb) wird insbesondere in der Anfangszeit in Österreich dringend benötigt. Nach der Phase der Orientierung und des Fußfassens in Österreich spiegelt sich die Reziprozität der Netzwerke unter anderem in der Unterstützung von Neuankömmlingen aus der eigenen ethnischen Community wider. Es lässt sich allgemein eine Offenheit für neue Perspektiven und multikulturell ausgerichtete Netzwerke erkennen, welche teils auch bewusst gewählt werden, um sich von der eigenen ethnischen Community zu distanzieren und damit sozialen Druck hinsichtlich normkonformen Verhaltens zu vermeiden. Je nach aktueller Lebenssituation und individuellen Bedürfnissen lässt sich eine Veränderung der Intensität und Unterschiedlichkeit der sozialen Kontakte erkennen, denn: "Networks of weak ties are flexible, practical, tactical and adaptive, but may not be durable.” (WiLliams 2006, p. 876). Die Einbindung in unterschiedliche Netzwerke offeriert den Respondent/inn/en vielseitige Möglichkeiten und Ressourcen, wie z.B. die Wohnungsvermittlung oder den Transfer von praktischen Informationen durch andere Afghan/inn/en, Hilfe beim Spracherwerb durch Österreicher/innen oder die Eröffnung neuer Perspektiven durch Angehörige anderer Minderheitengesellschaften. Beziehungen zu unterschiedlichen Personengruppen und damit verbundene positive Erfahrungen können schließlich ein Gefühl der Zugehörigkeit entstehen lassen bzw. verstärken (vgl. auch LiN 2001; DeINDL 2005). Trotz der erheblichen Relevanz und positiven Bewertung von sozialen Beziehungen $\mathrm{zu}$ Personen der österreichischen Mehrheitsgesellschaft hat sich die Wichtigkeit von Beziehungen zur eigenen ethnischen Community gezeigt. Ein kollektiver Erfahrungshintergrund und ein gemeinsamer kultureller Kontext können in einer neuen Lebenssituation für sozialen Halt und ein Heimatgefühl sorgen. Die Verpflichtung, an wichtigen Lebensereignissen von Angehörigen der eigenen ethnischen Community teilzuhaben, hat sich auch bei jenen gezeigt, welche sich bewusst von dieser Personengruppe distanzieren. Die starke Einbindung in Netzwerke der afghanischen Community, welche nicht getrennt von transnationalen Kontakten betrachtet werden kann, wird somit deutlich.

\subsection{Wertvorstellungen, Einstellungen und Handlungsregeln}

\subsubsection{Gender Roles und Genderbeziehungen}

\subsubsection{Gender als Schlüsselkategorie - Veränderungsprozesse der Wertvorstel- lungen und Erwartungshaltungen}

Bundesweit lebten in Österreich am 01.01.2019 43.073 Afghan/inn/en. Der Frauenanteil dieser Immigrant/inn/engruppe lag bei 32\% (vgl. StATISTIK Austria 2020). Aufgrund der unterschiedlichen, kulturell determinierten Geschlechterrollen 
in Afghanistan und Österreich legt die österreichische Politik ein Hauptaugenmerk auf die Emanzipation der afghanischen Frau, die im Rahmen eines strukturellen und sozialen Integrationsprozesses erfolgen soll. Dabei werden jedoch nicht nur emanzipierteres Verhalten, sondern auch ein Hinterfragen und ein Wandel der kulturell bedingten Geschlechterrollen und Geschlechterverhältnisse angestrebt. Das folgende Kapitel widmet sich der Rolle der afghanischen Frau in Österreich, den damit zusammenhängenden Rechten und Pflichten des Frauseins sowie den Veränderungen und Einflussfaktoren von Geschlechterrollen und Geschlechterverhältnissen, die Flucht und Aufenthalt im Aufnahmeland mit sich bringen.

Im Sinne einer eingehenden Analyse der qualitativen Daten ist Gender als Kategorie nicht nur im Zusammenhang mit diversen Fluchtgründen, sondern auch als wichtige Variable für den Verlauf integrativer Prozesse heranzuziehen. Obwohl bei der Formulierung der Genfer Flüchtlingskonvention (GFK) die Kategorie „Geschlecht“ ausgeklammert wurde, stellt sie einen wichtigen Faktor im Rahmen der Fluchtursachen dar. Fluchtgründe differieren zwischen den Geschlechtern und stehen in einem engen Zusammenhang mit der ökonomischen, sozialen und kulturellen Rolle der Frau im Herkunftsgebiet. Gesellschaftliche Strukturen im Herkunftsland können somit relevante Fluchtmotive darstellen, da sie für Frauen eine erhebliche Benachteiligung darstellen, wie z.B. Zwangsheirat (,arranged marriage"), Gewalt in der Ehe, außereheliche Schwangerschaften, sexuelle Übergriffe etc. Frauen sind daher spezifischen Formen der Verfolgung ausgesetzt, die auch im Rahmen des Integrationsprozesses in der Aufnahmegesellschaft nicht außer Acht gelassen werden dürfen (BINDER 2004). Gender repräsentiert somit eine Schlüsselkategorie im Rahmen der Analyse von Prozessen, die in einen ökonomischen, politischen, sozialen und kulturellen Kontext eingebettet sind und mit bestimmten Machtverhältnissen einhergehen (BINDER 2004; LuTZ 2010). Eine Auseinandersetzung mit Geschlechterrollen und -verhältnissen in Herkunfts- und Aufnahmeländern kann anhand von sozialen Praktiken erfolgen, die einander gegenübergestellt werden und die im Interesse einer österreichischen Integrationspolitik aufzeigen, ob und welche Veränderungen stattfinden und welche Einflussfaktoren eine übergeordnete Rolle spielen. Eine erfolgreiche Integration in Österreich impliziert die rasche Selbsterhaltungsfähigkeit sowie ein aktives Bemühen um das eigene Fortkommen und das Sich-Einbringen in die österreichische Gesellschaft (BMEIA 2015; EXPERTENRAT FÜR Integration 2017). Roggeband und Verloo (2007) sowie Kofman et al. (2015) thematisieren ungleiche Geschlechterverhältnisse in Minderheitsgesellschaften als zentrales Problem des Integrationsprozesses. Die oftmalige politische Zuschreibung der männlichen Täter- und weiblichen Opferrolle kollidiert mit den divergierenden kulturspezifischen Vorstellungen im Herkunfts- und Aufnahmestaat. Der „Status quo der Integration“ einer Community wird daher nicht nur anhand von finanzieller Selbständigkeit und gesamtgesellschaftlichem Mitwirken gemessen, sondern auch am Grad der Integration und Emanzipation migrierter Frauen. Die Emanzipation der Migrantin wird als Schritt „,nach vorne“ hin zu einer generellen Emanzipation der „migrant community“ interpretiert. Die geschlechtsspezifische Differenzierung von ökonomischen und sozialen 
Rahmenbedingungen im Asylland und die damit assoziierten Voraussetzungen, die für eine erfolgreiche Integration erforderlich sind, verstärken den Bezug zur Analysekategorie Gender im Integrationsprozess.

Die Migration afghanischer Frauen nach Österreich geht mit hinzugewonnenen Rechten einher. Nicht nur der im Sample wiederholt genannte Sicherheitsfaktor trägt $\mathrm{zu}$ einer durchwegs als besser bewerteten Lebensqualität bei, es ergeben sich auch neue Möglichkeiten der Mitwirkung am gesellschaftlichen Zusammenleben. Der erste Abschnitt der vorliegenden Analyse erläutert wie afghanische Asyl- und Schutzberechtigte die österreichische Gesetzes- und Rechtslage bewerten und welche Auswirkungen diese auf tradierte Geschlechterkonzepte hat. Der Fokus liegt auf einschränkenden Verhaltensnormen, welche die Gewalt an Frauen sowie Verhüllungsvorschriften betreffen. Das Subkapitel „Einstellung zu Bildung“ skizziert Einstellungen bezüglich der strukturellen Einbindung ins österreichische Bildungssystem, wie etwa zum Bildungszugang beider Geschlechter und zum koedukativen Unterricht. Des Weiteren werden Zukunftspläne von Eltern sowie ein daraus resultierender bildungsbedingter Gewinn an emanzipiertem Denken betrachtet. Sprach- und Bildungserwerb repräsentieren im Rahmen der strukturellen Integration primär relevante Faktoren, die den Frauen den Weg zu einer autonomeren Lebensweise ebnen. Jedoch weisen Afghaninnen heterogene Voraussetzungen auf, die auf unterschiedliche sozioökonomische Realitäten zurückzuführen sind (z.B. Herkunft, kultureller, politischer sowie biographischer Hintergrund, Alter, sozioökonomischer Status, Aufenthaltsdauer, Bildungsniveau) und ein erwünschtes „integratives" Fortschreiten innerhalb der Aufnahmegesellschaft beeinflussen (BINDER 2004; ZULFACAR 2006). Diese Voraussetzungen variieren nicht nur innerhalb heterogener Frauengruppen, sondern auch stark zwischen den Geschlechtern. Während Männer in Afghanistan eine ökonomisch und sozial privilegierte Rolle einnehmen, sind die Rechte der Frauen eingeschränkt (vgl. BFA 2017). So ist traditionell der alltägliche Arbeitsbereich der Männer außerhalb des Hauses verortet, während Frauen für die Hausarbeit zuständig sind. Die geschlechterspezifischen Aufgabenverteilungen und Verantwortungsbereiche müssen zwar nach Herkunftsgebiet (urbaner bzw. ländlicher Raum) und Bildungsstatus differenziert werden, jedoch existiert eine generalisierte kulturelle Geschlechternorm, die patriarchale Familienstrukturen legitimiert und aufrechterhält. Wie sich Haushaltsstruktur und Aufgabengebiete (v.a. Kindererziehung und Haushalt) innerhalb des familiären Zusammenlebens im Asylland zwischen den Geschlechtern verteilen und welche Einflussfaktoren für veränderte Zustände signifikant sind, wird im einleitenden Abschnitt „Haushalt und die familiäre Aufgabenteilung“ anhand der sozialen Determinanten analysiert. Einen weiteren ausschlaggebenden Faktor stellt die afghanische Community in Österreich dar, d.h. geschlechtsspezifischen Erwartungshaltungen, die aufgrund der generationsbezogen differenten kulturellen Sozialisierung zu Konflikten führen kann. Die Interaktionen mit der österreichischen Mehrheitsgesellschaft führen einen generationstypischen Anpassungsprozess weiter und zeigen, dass vor allem junge Afghan/inn/en aufgrund der raschen strukturellen (Schulbesuch/ Lehre) und sozialen (Freizeit- und Sportprogramme) Einbindung einem österreichi- 
schen normativen Verständnis von gelebter Gleichberechtigung eher nachkommen als ihre in Afghanistan sozialisierten Eltern. Auch die Wahl der (Ehe-)Partner/innen und die damit assoziierten Erwartungshaltungen der Eltern, der „extended family“ im Herkunftsland sowie der afghanischen Community in Österreich nehmen Einfluss auf geschlechterkonformes Verhalten. Im Zentrum stehen hier die Bedeutung der religiösen und ethnischen Zugehörigkeit der potentiellen (Ehe-)Partner/innen, die damit verbundenen geschlechterspezifischen Toleranzspielräume der Eltern und die Wahrnehmung der Konsequenzen, die mit einer individuellen Entscheidungsfindung einhergehen. Im dritten und letzten Analysekapitel werfen Zitate der Respondent/inn/en einen Blick auf geschlechtsspezifisches Integrationsverhalten.

\subsubsection{Die Frau und ihre Rechte (Gesetzeslage und Normen in Österreich)}

Die Analyse zeigt, dass eine große Mehrheit der Befragten die aktuelle Rechtslage in Österreich begrüßt und als Gewinn für die soziale Rolle der Frau bewertet. Patriarchal- traditionelle Gesellschaftsstrukturen des Herkunftslandes werden überwiegend kritisiert und auf eine „falsche“ Auslegung des Islams zurückgeführt. Diese wird von einigen Respondent/inn/en als Grundlage einer Rechtszuschreibung bezüglich des Status und der Rechte der Frau in Afghanistan interpretiert. Des Weiteren wird betont, dass der Aufenthalt im Asylland eine Anpassung an rechtliche Rahmenbedingungen erfordert. Anpassungsmechanismen und Bewältigungsstrategien variieren jedoch innerhalb der afghanischen Gemeinschaft und werden geschlechterspezifisch ausgelegt. Obwohl eine überwiegende Mehrheit des Samples die gesetzlichen Rahmenbedingungen in Österreich nicht hinterfragt, wird von einer Minderheit mit hohem Bildungsstatus betont, dass eine Gleichstellung von Mann und Frau auch in Österreich nicht gegeben ist.

„Islam wird öfter falsch verstanden. Wenn ich so denke, dann denke ich mir, dass hier [in Österreich] die Menschen sehr viele Rechte haben, vor allem sind die Rechte von Frauen und Männern gleich. Im Islam gibt es auch Frauenrechte. In Afghanistan und in manchen anderen Staaten gibt es keine Frauenrechte, dort ist die Lage nicht gut. Es gibt einen großen Unterschied zwischen Österreich und Afghanistan. In Afghanistan herrscht eine patriarchalische Gesellschaft, Frauen haben keine Rechte. Im wahren Islam gibt es Gleichberechtigung zwischen Frauen und Männern, was es auch hier in Österreich gibt. Eine Frau kann sich in Österreich als Mensch bzw. Frau sehen, aber in anderen „finsteren" Staaten dürfen Frauen nicht einmal das Haus verlassen und auch nicht arbeiten. " (I57, weiblich, seit 2015 in Ö.)

„Frauen und Männer sind gleichberechtigt. Die Rechte, die ich habe, hat auch eine Frau und die Rechte, die eine Frau hat, habe ich auch. Denn wenn eine Frau arbeiten geht, zahlt sie Steuern, sie ist versichert und das, was sie kann, ist nicht viel anders als das, was ich kann. Daraus folgt, dass Frauen und Männer gleich sind. Und da ich hier in Österreich lebe, denke ich auch wie ein Österreicher. Ich kenne aber auch Afghanen, die nicht so denken und noch so denken, als wären sie 
noch in Afghanistan. Jene, die noch nicht die Sprache gelernt haben, sich noch nicht nach einer Arbeit umgesehen haben. “ (I63, männlich, seit 2001 in Ö.)

„Für mich sind Frauen und Männer gleichgestellt und niemand darf der/dem anderen Befehle erteilen und sagen was sieler zu tun hat. Nicht so wie in Afghanistan, wo die Frauen das Eine machen und das Andere nicht machen dürfen. Hier gibt es Menschenrechte und herrscht die Demokratie. Aber wenn man die Österreicher/innen fragt, sagen sie auch, dass auch hier in Österreich diese Menschenrechte nicht eingehalten werden. Aber im Vergleich zu den asiatischen Ländern ist es hier besser. Meiner Meinung nach, müssen alle gleichgestellt sein und beide sind ja Menschen und haben das Recht zu leben. " (I54, männlich, seit 2015 in Ö.)

\subsubsection{Gewalt gegenüber Frauen}

Die aktuelle Sicherheitslage sowie die Gewalt gegen Frauen sind wichtige Fluchtmotive, die afghanische Frauen dazu bewegen, ihre Heimat zu verlassen. Obwohl die afghanische Gesetzgebung die Rechte der Frau in einem historischen Kontext unterschiedlich auslegt und es Frauen auch gesetzlich phasenweise möglich war/ist, von ihren Rechten Gebrauch zu machen, suchen viele Frauen im Falle häuslicher Gewalt oder sexueller Misshandlung kaum rechtliche Unterstützung. Dies liegt einerseits daran, dass Frauen ihre Rechte nicht kennen und andererseits, weil sie Verfolgung befürchten und/oder Angst davor haben, wieder zu ihren Familien bzw. den Tätern zurückgeschickt zu werden. Des Weiteren werden unbegleitete Frauen im öffentlichen Raum vermehrt Opfer von Übergriffen (BFA 2017). Patriarchale Familienstrukturen legitimieren eine männliche Dominanzposition, die u.a. über physische Gewalt eingefordert wird. Frauen werden so nicht nur im Herkunftsland Opfer von Ehrenmorden, die Tragweite von kulturell legitimiertem Verhalten ist auch im Asylland (vgl. Der Standard 2018) gegeben. Politisch festgeschriebene Integrationsmaßnahmen, wie z.B. jene im Rahmen der vom ÖIF durchgeführten Werte- und Orientierungskurse, besitzen zwar aufklärenden Charakter, jedoch ist ein Konsens über die gewonnenen Rechte für afghanische Frauen stark von der Dauer des Aufenthalts im Asylland abhängig. Veränderungen der familiären Strukturen stehen in einem Konnex zu rechtlichen Sanktionen gegen gewaltbehaftete männliche Verhaltensmuster sowie zum Anspruch der Frauen auf den Bezug von Sozialleistungen. Eine Respondentin schildert:

„Mein Mann ist anders geworden jetzt in Österreich. Gott sei Dank anderer geworden. Und ich bekomme jeden Monat vom Sozialamt auf mein Konto und das Kindergeld auch auf mein Konto. Ich zahle die Wohnungsmiete jeden Monat selber, mein Mann macht das nicht. Und ich zahle den Strom und Geld oder Erlagscheine für dieses UPC oder sowas. Ich zahle alles. Und das Geld alles kommt auf mein Konto. Ich entscheide, was wir kaufen, was möchten wir kaufen oder was möchten wir zum Leben haben. Mein Mann kann das nicht entscheiden, weil er weiß das ist Österreich. Die Frauen haben viele Rechte und Frauen sind sehr 
schlau. Sie machen immer Rechte. Und mein Mann sagt nur: ,Du kannst nur mehr ein bisschen Essen geben oder für mich eine Kleidung bringen oder so. 'Er macht nichts mit mir, Streit oder sowas. " (I19, weiblich, seit 2011 in Ö.)

Kommt Gewalt in der Ehe vor, so dürfen in Österreich auch Frauen einen Scheidungsantrag stellen, was die Mehrheit der Befragten befürwortet.

„I: Und würdet ihr z.B., wenn eure Töchter jetzt einen Mann hätten, der sie schlagen würde oder der jetzt nicht gut für sie ist, und sie möchte dann weg, würdet ihr sie unterstützen und sagen: ,Ja, gehe weg von dem Mann und lebe alleine?' B: Ja, weil das ist ihr Recht. Wenn der Mann eine Schlägerei macht, warum soll eine Frau dann bleiben? Ja, das ist ein Recht. Und ich finde, das ist gut in unserem Land [in Österreich], dass man das Recht hat sich scheiden zu lassen. [In Afghanistan] müssen die Männer sagen ,du bist geschieden geworden'. Weil die Frau hat viele Probleme, aber trotzdem kann sie das nicht sagen. Aber in Österreich haben auch Frauen dieses Recht. Das freut mich. Eine Frau kann von ihrem Mann geschieden werden oder alleine leben und alle Kinder nehmen, zusammenleben, ja. Und es kommt das Geld von der Regierung und das ist auch sehr gut und das unterstützt die Frauen und Kinder. Und die Frauen haben viele Rechte hier in Österreich. “ (I20, weiblich, seit 2013 in Ö.)

\subsubsection{Verhüllung und Freizügigkeit}

Das am 01. Oktober 2017 erlassene Anti-Gesichtsverhüllungsgesetz (AGesVG) schreibt als Bundesgesetz ein Verhüllungsverbot vor, das bei Nichtbeachtung mit einer Verwaltungsstrafe sanktioniert wird. Ziel ist dabei, dass Gesichtszüge mittels Kleidung oder anderer Gegenstände nicht verborgen werden dürfen, sodass ein Erkennen derselben gewährleistet ist (DiAKONiE 2017; BunDESKANZLERAMT 2018). Zwar lehnt eine überwiegende Mehrheit männlicher und weiblicher Befragter eine Vollverschleierung zur Gänze $\mathrm{ab}$, Einstellungen bezüglich der Verhüllung von Frauen differieren jedoch nach Alter, Bildungsstatus, Religiosität, Aufenthaltsdauer in Österreich etc.

„Burka ist ... nicht im Islam. Das ist nicht gut. Und ja, ich mag das nicht die Burka. Das ist nicht schön für eine Frau. Kopftuch das ist etwas Anderes. Aber Burka das ist ja schlimm. Das ist gut, dass es ein Verbot für die Burka gibt. Ich bin auch gegen Burka. Was ist das? Das ist nicht im Islam eigentlich. Ich habe im Koran in allen Büchern [nachgesehen], ich habe nichts gefunden ... Im Islam ist das nur ein Kopftuch, das Frauen tragen müssen. Ja, aber wenn es jemand nicht trägt, das ist auch Freiheit, ja. (I26, männlich, seit 2013 in Ö.)

Das Verhüllen bestimmter Körperteile bringt auch Einschränkungen mit sich. Diese werden vor allem im Zusammenhang mit der Arbeitssuche erwähnt.

„,Mit meinem Kopftuch kann man nicht arbeiten, in vielen verschiedenen Bereichen. Aber ich kämpfe. I: Aber ist es dir wichtig ein Kopftuch zu tragen? B: Ja, es ist mir wichtig. " (I24, weiblich, seit 2015 in Ö.) 
Das Tragen eines Kopftuchs wird von einer überwiegenden Mehrheit als Privatsache gesehen. Insbesondere zu bestimmten Anlässen (wie z.B. Feierlichkeiten, Moscheebesuche etc.) heben Befragte die Relevanz desselben hervor.

„B: ... meine Mutter trägt auch kein Kopftuch. I: Auch nicht? B: Auch nicht. ... sie sagt immer, dass, als es in Afghanistan keine Taliban gab, hatte ich kein Kopftuch auch dort in Afghanistan und sie fühlt sich wohl ohne Kopftuch. Wenn sie betet, trägt sie ein Kopftuch und ansonsten trägt sie kein Kopftuch. " (I18, weiblich, seit 2016 in Ö.)

Des Weiteren ist das Tragen eines Kopftuchs abhängig von der Dauer des Aufenthalts im Asylland. So stößt nach längerem Verbleib in Österreich ein Ablegen der Kopfbedeckung auf verstärkten Zuspruch.

„, Was mir ganz stark auffällt ist, dass bei den Afghaninnen irgendwie ... das Kopftuch rutscht immer weiter nach hinten, also je länger sie da sind. “(E5)

„Ich habe eine Freundin, sie will Kopftuch tragen und ich respektiere das. ... ich habe auch am Anfang Kopftuch gehabt und ich hab' gedacht, so ist es besser. Dann [wurde das Kopftuch abgelegt] meine Familie hat auch kein Problem damit gehabt. Mein Mutter trägt es, aber ich nicht. " (I44, weiblich, seit 2015 in Ö.)

Eine Minderheit der Befragten sieht das Anti-Gesichtsverhüllungsgesetz als Widerspruch zu demokratischen Werten, denn diese beinhalten die Meinungs- und Religionsfreiheit und die Freiheit sich so zu kleiden, wie man möchte:

„, Gestern wurde diesen vier Frauen im Flugzeug von Dubai im Flughafen Wien sofort gesagt, dass sie die Burka herunternehmen müssen und nur Kopftuch [tragen dürfen]. ... Und da bin ich auch dagegen. Freiheit leben heißt nicht, dass du mit dem Bikini auf die Straße gehst. Freiheit bedeutet für mich ... freies Leben, freie Bewegung. Mach was du willst, nur nicht dich umbringen, nicht jemanden verletzen, wie ich vorher gesagt habe. Aber das Burkaverbot das kommt wieder von der Politik, ... das wird wieder im Parlament gestempelt und der Oberste Gerichtshof sagt ja. Also dann ist alles Politik, es gibt keine Religion ohne Politik. “ (I27, männlich, seit 2005 in Ö.)

Einstellungen zu weiblichen Verhüllungsvorschriften hängen auch vom Zeitpunkt der Zuwanderung und den politischen Verhältnissen in Afghanistan ab. Frauen, die in der sowjetischen Ära Afghanistans aufgewachsen sind, lehnen Verhüllungsgebote tendenziell stärker ab.

„Ja, muss langen Rock. Aber früher, 50 Jahre früher, meine Mutter hat Minirock getragen. Und sie hat ein Foto gemacht. Sie hat einen Minirock, ohne Kopftuch und so, nur eine Bluse mit Minirock ohne Strumpfhose. ... Sie hatte viele Freiheiten. Aber später, als diese anderen Leute in Afghanistan gekommen sind, war alles kaputt. Meine Mutter hat manchmal über mich gelacht und sagte: ,Du hast Burka getragen, aber ich habe Minirock gehabt' [lacht]. Ja, aber das ist so, wenn die 
Welt ein bisschen weiter geht. Die Kinder müssen besser werden als ihre Mutter oder Vater oder als Großmutter oder so. Aber bei uns ist es so ... unsere Großen [Eltern] hatten Freiheit, aber wir hatten Burka und Kopftuch. " (I20, weiblich, seit 2013 in Ö.)

„In Afghanistan haben die Frauen keine Rechte, hier [in Österreich] kann jede das Anziehen was sie will, mit jedem sprechen. Sie wissen es ja besser. ... Dort war es echt schwierig, hier bin ich aber frei. Kann überall hingehen und auch allein. Dort musste mich mein Mann begleiten, wenn ich wohin wollte. Oder irgendwer hat mich immer gezwungen, meine Burka oben zu haben. Es war schwierig für mich. Ich habe in der UdSSR studiert und bin dort aufgewachsen. Ich habe nie eine Burka getragen von Kind an. Für die Frauen hier ist es viel besser geworden. Für die Männer weiß ich es nicht, aber für die Frauen bestimmt. " (I28, weiblich, seit 2013 in Ö.)

Vor allem die jüngere Generation fühlt sich verpflichtet, den Erwartungshaltungen von Familie und Community zu entsprechen. Das Tragen eines Kopftuchs wird zwar innerfamiliär nicht zwangsweise auferlegt, jedoch wird auf diese Weise einer damit assoziierten Rolle der „guten Muslimin“ entsprochen.

„I: Und Ihre Töchter [tragen das Kopftuch] auch nicht? B: Nein, sie sicher nicht. In Europa ist geboren ..., aber die erste Tochter schon jetzt. Nicht ganz so ..., aber ein bisschen zugedeckt ohne Schleier ... Durch den Mann, ihr Mann mag das nicht. Weil er in Moschee arbeitet oder ist beteiligt oder Mitglied keine Ahnung. Aus diesem Grund muss seine Frau auch ein bisschen zurückhaltend sein. Weil alle Leute sagen: ,Ah, ihr Mann ist in der Moschee, betet fünf Mal, und schaust du seine Frau an, bitte. ' ... aber alle meine Töchter tragen keinen Schleier. “ (I33, männlich, seit 1974 in Ö.)

„I: Hätte deine Mama gerne, dass du hier auch Kopftuch trägst? B: Anfangs wollte sie schon, als ich z.B. 19 Jahre alt war, wollte ich schon. Da hat sie gemeint: ,Du bist schon ein großes Mädchen, das darf nicht sein. Man muss sich bedecken." $J a$, solche Sachen. Aber mein Vater war eher lockerer. Dadurch, dass mein Vater lockerer war, mussten wir nicht, ich und meine Schwester." (I15, weiblich, seit 2001 in Ö.)

Verhüllungsgebote sind u.a. ein Kausalfaktor für eine konfliktbehaftete ElternKind-Beziehung. Zwischen den Generationen bestehende Einstellungsunterschiede sind überwiegend auf Einflüsse des österreichischen Bildungssystems zurückzuführen und offenbaren ein Aufeinanderprallen unterschiedlicher Wertesysteme. Die soziale Konfrontation afghanischer Jugendlicher mit den Gleichaltrigen der österreichischen Mehrheitsgesellschaft trägt dazu bei, dass Heranwachsende nicht aufgrund ihres „,nonkonformen“ Verhaltens in der Gruppe auffallen wollen.

„Meine Mutter hat nicht oft darüber geredet zu Hause und es auch nicht angesprochen. Vielleicht wusste sie, dass wir eh das Kopftuch tragen in der 
Schule und auch so brav sind wie sie selber und andere Mädchen im Iran. Meine Schwester hat es immer getragen, aber ich habe es nicht getragen im Sportunterricht. Und meine Schwester hat es nicht zuhause erzählt und ich habe auch nicht davon erzählt ... Wenn ich nichts sage, dann ist der Vater dagegen. Ich habe zu meiner Cousine darum gesagt, sie soll einfach nichts sagen. Das ist das beste. Dann wird es zuhause Ruhe geben. Ich habe es immer so gemacht und es war bis jetzt kein Problem. " (I13, weiblich, seit 2008 in Ö.)

„, Aber als ich älter war, ich war, glaube ich, in der 3. [Klasse] Gymnasium, wollte ich Volleyball spielen. Nein, zuerst Handball. Ich war auch ein Jahr im Handballteam, da war es auch so. Soll ich jetzt weiterspielen oder nicht, weil die ziehen solche Sachen an? Ich habe immer längere Sachen getragen und meine Kolleginnen haben mich gefragt, wieso ich das mache. Und es war mir auch unangenehm kurze Sachen zu tragen und auch lange Sachen. " (I15, weiblich, seit 2001 in Ö.)

\subsubsection{Einstellungen zur Bildung}

Afghanistan hat eine geschätzte Alphabetisierungsrate von 31\% - eine der niedrigsten der Welt - und diese weist eine große Schwankungsbreite zwischen Männern und Frauen sowie städtischen und ländlichen Gebieten auf. Während die weibliche Alphabetisierungsrate im urbanen Raum bei 40\% liegt, jene der Männer bei 69\%, kann in ruralen Gebieten nur ein Fünftel der Bevölkerung Lesen und Schreiben, 10\% davon sind weiblich und 39\% männlich (AHMAD 2017, p. 4 f.).

Die ungleiche Verteilung der Lese- und Schreibkompetenzen zwischen den Geschlechtern ist auf eine Kombination verschiedenster Faktoren zurückzuführen. Die kulturelle Norm untersagt den Mädchen und Frauen den Erwerb eines höheren Bildungsstatus, ein Schulbesuch kollidiert mit Pflichten, die ein/e Mädchen/Frau im häuslichen Bereich zu erfüllen hat. Des Weiteren spielt die nicht zu gewährleistende Sicherheit auf dem Schulweg eine Rolle, die sich auch im Stadt-Land-Gefälle wiederspiegelt (ebd.). Die Schulbildung der Mädchen in Afghanistan hat eine historische Wandlung erfahren. Frauen, die der sowjetischen Ära Afghanistans entstammen, sind mit einer geschlechtergemischten Unterrichtsstruktur vertraut und weisen einen höheren Bildungsstatus auf als jene, die unter der Taliban-Herrschaft herangewachsen sind. So bringen Frauen, die in den 1970er- und 1980er-Jahren emigriert sind, vermehrt berufliche Qualifikationen mit, die eine Eingliederung in den österreichischen Arbeitsmarkt erleichtern. Insbesondere geht der Erwerb der deutschen Sprache rascher voran, bzw. besteht ein stärkeres Bemühen, sich sprachliche Qualifikationen anzueignen. Die Wichtigkeit von Schulbildung, im Speziellen jener der Töchter, wird besonders betont. Die Interviews bestätigen, dass die Einstellungen und Erwartungshaltungen der Eltern zum Bildungszugang ihrer Töchter und Söhne je nach Zuwanderungszeitpunkt, Bildungsstatus, regionaler Herkunft etc. variieren:

„,Weil oft, wenn man von so was [Arbeitsmarktintegration] redet, dann denkt man: ,Ok, aus religiösen Motiven werden die Frauen sozusagen ferngehalten. 'Es ist 
aber nicht so. Ich habe oft Leute gehabt, die vollen Hijab hatten und trotzdem bei mir in der Beratung waren und wollten in einen Deutschkurs und auch andere Maßnahmen besuchen. Also ich würde das jetzt nicht grad mit der Religion und Überzeugung oder so verbinden, sondern eher, wenn dann nur mit Alter und mit dem Bildungsniveau. "(E6)

Auch in den Interviews mit den Respondent/inn/en hat sich gezeigt, dass Einstellungen und Erwartungshaltungen der Männer und Frauen bezüglich des Bildungszugangs ihrer Töchter und Söhne je nach Zuwanderungszeitpunkt, Bildungsstatus, regionaler Herkunft etc. variieren. Eine (Aus-)Bildung im Allgemeinen aber wird von den Befragten für beide Geschlechter als erstrebenswert betrachtet.

„Natürlich befürworte ich Ausbildung für Frauen und Männer. Für beide Geschlechter sollte es dieselben Rechte geben. Ich bin dafür, dass alle meine Kinder eine Ausbildung haben. Ich habe selbst in Afghanistan studiert, damals haben Mädchen und Buben gemeinsam gelernt, sowohl in der Schule als auch auf der Universität, aber heute dürfen sie das nicht. Sie lernen in getrennten Gebäuden. Zur Zeit meiner Jugend hat es bessere Zeiten und Frauenrechte gegeben. Aber als die Mujaheddin und die Taliban gekommen sind, haben Frauen keine Rechte mehr gehabt. Sie zwangen die Frauen, nicht zu studieren. " (I57, weiblich, seit 2015 in Ö.)

\subsubsection{Koedukativer versus geschlechtergetrennter Unterricht}

Die Befragten plädieren für koedukativen Unterricht und gleichberechtigte Bildungsmöglichkeiten. Gemischte Klassen werden als positiver Faktor für ein gesamtgesellschaftliches Miteinander angesehen. Die Koedukation soll das Verständnis für das jeweils andere Geschlecht fördern.

„I: Und was halten Sie davon, dass hier Burschen und Mädchen in eine gemeinsame Klasse gehen? B: Nun sowas ist hier normal. So ist es in dieser Kultur. Ich persönlich finde das auch gut, denn so gewöhnen sie sich aneinander und lernen einen guten Umgang miteinander. “ (I65, männlich, seit 2015 in Ö.)

„Aber hier, wenn man so gemischt ist, da fängt die Gleichberechtigung schon an. Wenn man so anfängt, stellt man die Gleichberechtigungsfrage, wenn man erwachsen ist, gar nicht. Das ist automatisch ... wenn es gemischt ist. Die Kinder wachsen damit auf, dass eigentlich alle gleich sind. Es gibt keine Unterschiede. “ (I4, männlich, seit 2001 in Ö.)

Lediglich ein männlicher Respondent spricht sich zwar für Koedukation in der Schule aus, stellt jedoch eine gleichberechtigte Lebensweise von Männern und Frauen in Abrede.

„,[Frau übersetzt für ihren Mann] Er meint, er findet es gut wenn eine Frau in die Schule geht. Aber, dass wir halt wie die Österreicher [unverständlich] deren Kultur, Frau-Mann-Gleichberechtigung [leben]. Er meint es geht nicht. ... Ich 
weiß nicht, ob er meint, es ist nicht möglich oder dass es einfach nicht geht. “ (I43, männlich, seit 2012 in Ö.)

\subsubsection{Sport- und Schwimmunterricht}

Die Verhüllungsdebatte nimmt auch bei sportlichen Aktivitäten eine zentrale Rolle ein. Wie unsere Studie zeigt, differieren elterliche Erwartungshaltungen zwischen Sportund Schwimmstunden. Während im Sportunterricht der jugendlichen Kleiderwahl größere Toleranz entgegengebracht wird, spricht sich eine überwiegende Mehrheit für bedeckte Kleidung in den Schwimmstunden aus. Gründe dafür sind Religiosität sowie üble Nachrede bei nicht entsprechendem Verhalten innerhalb der Community. Die Ambivalenz, sowohl österreichischen als auch afghanischen Verhaltensregeln zu entsprechen, ist für junge Frauen eine Herausforderung. Während manche Mädchen ein Unbehagen verspüren, sich im Bikini oder Badeanzug zu zeigen, bemühen sich andere wiederum, ihre „,verbotene Freizügigkeit“" vor den strengen Blicken traditionell orientierter Eltern geheim zu halten.

„,Ich gehe nicht baden, ehrlich gesagt. Nein, gehe ich nicht. Meine Schwester geht. Aber man muss dazu sagen, sie hat so ein Dings an, so ein T-Shirt, und dann so eine kurze Hose. I: Also kein Badeanzug oder Bikini, sondern so Sportkleidung? B: Genau! Da ist meine Mama voll streng. Mein Papa nicht so, ... aber meine Mama ist einfach total [streng], weil wir die Töchter sind einfach. "(I14, weiblich, seit 2001 in Ö.)

Der koedukative Unterricht im Rahmen von sportlichen Aktivitäten erfährt hinsichtlich der Schwimmstunden (bei Eltern) vermehrte Ablehnung.

„, Was das Schwimmen angeht, bin ich ehrlich gesagt noch unschlüssig, ob ich das gutheißen soll oder nicht, aber Sport geht für mich mehr als in Ordnung. In der Gesellschaft, in der wir heute leben, gibt es keine Geschlechtertrennung. ... Und so kann es auch in einer Schulklasse aussehen. Ich sehe darin überhaupt kein Problem. " (I63, männlich, seit 2001 in Ö.)

„B2: Und für mich zum Beispiel, ich werde meine Tochter schon in ein Schwimmbad lassen, aber da müssen Männer und Frauen schon getrennt sein, weil es im Islam so ist. Und das ist meine Religion. Meine Religion kann ich nicht vergessen. Egal, auch wenn ich in Österreich bin, egal, auch wenn ich hier geboren bin. Denn meine Religion ist Islam und im Islam darf man so was nicht. B1: Ja! B2: Und das will ich nicht. Und das lasse ich auch bei meiner Tochter nicht zu. " (I6, weiblich, seit 2003 in Ö.; I7, weiblich, seit 2001 in Ö.)

\subsubsection{Geschlechterspezifische Zukunftsvorstellungen der Eltern hinsichtlich der Ausbildung}

(Aus-)Bildungsmöglichkeiten im Asylland, die eine gendergerechte Ausgangsbasis für spätere berufliche Tätigkeiten ermöglichen, erfahren geschlechtsunabhängig großen Zuspruch. Besonders Mütter schreiben der Bildung einen hohen Stellenwert 
zu und wünschen sich für ihre Töchter ein unabhängiges und eigenständiges Leben. Grundtenor ist: „Sie sollen es besser haben als wir.“

„I: Und haben Sie für Ihre Kinder die gleichen Erwartungen oder Zukunftsvorstellungen für die Mädchen wie auch für die Buben, oder ist da ein Unterschied? B: Ja, die Kinder entscheiden selber, glaube ich. “(I26, männlich, seit 2013 in Ö.)

„I: Und haben Sie für das Mädchen, haben Sie da dieselben Zukunftsvorstellungen wie für die Burschen? B: Natürlich! Mehr als die Burschen. ... I: Warum mehr? B: Sagen wir mal, die Burschen sind von Natur aus stärker als Frauen. ... deshalb sage ich, der Frau oder dem Mädchen muss mehr geholfen werden als den Burschen. Die Burschen werden auch [unverständlich] körperlich was erreichen, wenn sie nicht anders etwas erreichen können. Aber eine Frau nicht." (I22, männlich, seit 1986 in Ö.)

\subsubsection{Bildung und emanzipiertes Denken}

Ein hoher Anteil der rezent nach Österreich geflüchteten Asyl- und Schutzberechtigten, v. a. jene der Jahre 2015 und 2016, weist ein tendenziell niedrigeres Bildungsniveau auf als die bereits seit Jahrzehnten in Österreich lebenden Afghan/inn/en. Vor allem junge Männer aus den ruralen Gebieten Afghanistans, die stark von den dort vorherrschenden traditionellen islamischen Werten geprägt sind, werden im Laufe eines strukturellen Integrationsprozesses (Schulbildung, Sprachkurs etc.) mit Einstellungen konfrontiert, die ein Hinterfragen traditioneller Denkmuster einleiten.

„Früher dachte ich anders, weil ich in einem Land lebte, in dem viele Unterschiede zwischen Mann und Frau herrschen. Männer haben immer gearbeitet und die Frauen waren nur zuhause. Seitdem ich in Europa lebe und hier in die Schule gehe und vieles erklärt bekommen habe, das mich überzeugt hat, denke ich anders. “ (I47, männlich, seit 2015 in Ö.)

„Ja, ich dachte früher anders. ... Im Sprachkurs sind zwei Frauen, die kommen und uns diese Dinge erzählen und spielen Theater, wie eine österreichische Familie ausschaut und lebt und wie eine afghanische. Dort habe ich kennengelernt, dass beide arbeiten müssen ... Ich scherze mit meinem Mann und sage es ihm dadurch, aber es ist immer noch so. Er arbeitet draußen und ich zuhause [lacht]. " (I28, weiblich, seit 2013 in Ö.)

\subsubsection{Verantwortungsbereiche im Haushalt und die familiäre Aufgabenteilung}

Die ökonomische und soziale Rolle der Frau in Afghanistan ist zwar abhängig vom Herkunftsgebiet (Stadt bzw. Land), generell kann aber davon ausgegangen werden, dass Frauen die Verantwortung für Haushalt und Kinder tragen, während Männer beruflich außerhalb des Hauses verortet sind. Abgesehen von Rollenzuschreibungen sind viele afghanische Frauen, die über eine Familienzusammenführung nach Österreich kommen, auf Hilfestellungen ihrer (Ehe-)Partner angewiesen. Anfängliche Sprachbarrieren, fehlende Arbeitsplätze sowie geringer sozialer Anschluss sind maßgebliche Faktoren, 
die einer autonomen Lebensweise entgegenwirken. Der erhebliche Zuspruch zu einem theoretischen Ideal der gleichberechtigten Arbeitsteilung wird in der Praxis häufig nicht umgesetzt. Oftmals werden die Haushaltsstrukturen innerhalb der Familie weitgehend wie im Herkunftsland weitergeführt.

„,Bei mir entscheidet oft die Frau [lacht]. Bei uns ist es, Gott sei Dank, viel besser als bei den anderen afghanischen Familien. Also bei den afghanischen Familien ich kann behaupten: Fast 90\% der Entscheidungen liegen in der Hand der Männer. Ja leider, das stimmt. " (I37, männlich, seit 1988 in Ö.)

„Nein, wenn ich meine Frau nach Österreich hole, muss sie arbeiten, weil ich nicht alleine für alles in der Familie aufkommen kann, [lacht] und solange sie nicht schwanger ist, muss sie die Sprache lernen und arbeiten. " (I54, männlich, seit 2015 in Ö.)

„Wir sind als Kind schon daran gewöhnt, dass die Männer draußen und die Frauen zuhause arbeiten [lacht]. Aber hier [in Österreich] ist es nicht so. Beide teilen sich die Arbeit auf. Das ist doch etwas Offensichtliches, aber die Männer machen es nicht. " (I28, weiblich, seit 2013 in Ö.)

Obwohl Frauen auf dem Arbeitsmarkt im Allgemeinen als ,integrierbar“ gelten, da sie für „einfache“ Hilfstätigkeiten leichter vermittelbar sind, treten aufgrund der strukturellen Voraussetzungen mehr Männer in den österreichischen Arbeitsmarkt ein. Da meist mehrere Familienmitglieder vom Einkommen des (in der Regel) Vaters abhängig sind, wird seinem Arbeitsplatz höchster Stellenwert zugeschrieben. Für die Hausarbeit ist somit jene Person zuständig, die über keine außerhäusliche Arbeitsstelle verfügt dies betrifft somit überwiegend Frauen.

„, Wenn ich nach Hause komme und sehe es ist schmutzig und es ist nicht alles in Ordnung, dann mach ich es halt, dann arbeite ich für meine Frau. Also ich helfe ihr sehr gerne, nicht nur meiner Frau, also Kinder, alles, wenn was ist, dann mache ich mit. Und jetzt bin ich ganz allein. Also ich mache es jetzt allein. Also ich putze, ich passe auf alle Kinder auf, ich koche ... " (I22, männlich, seit 1986 in Ö.)

„Ich gehe jetzt weg, weil ich arbeite. Wenn ich zuhause bin, dann mache ich alles. Ich habe eine eigene Wohnung, da muss ich putzen. Mein Mann macht nichts, da muss ich alles machen. Ich habe zwei Arbeiten. Meine Mutter wohnt hier, ich bin hier. Sie ist Stiege 34 und ich bin Stiege 39. Wir sind nicht so weit weg. Und ich hab 'zwei Arbeiten, ich putze hier und dann gehe ich Mama helfen. Und dann gehe ich arbeiten. Und ein Kind habe ich auch. " (I7, weiblich, seit 2001 in Ö.)

„,... die Frauen sind nicht für schwere Arbeit geschaffen und es ist besser, dass sie die leichte Arbeit im Haus erledigen. ... Meine Meinung ist es, dass die Erziehung der Kinder im Kindesalter mehr die Aufgabe der Frau ist. Aber auch die Männer müssen sich einbringen, aber mehr ist es von den Frauen. Und die Arbeit ist 
grundsätzlich für die Männer; ich meine die schwere Arbeit. “ (I47, männlich, seit 2015 in Ö.)

\subsubsection{Kindererziehung}

Kindererziehung und Hausarbeit sind nicht nur in afghanischen Familien, sondern überwiegend auch in Österreich, Frauensache. Auch hier gilt: Die besserverdienende (meist männliche) Person sorgt für finanzielle Sicherheit, während in der Regel der weibliche Part die Erziehung der Kinder übernimmt. Die Analyse des Samples zeigt jedoch, dass sich die Partizipation der Geschlechter an Erziehungsmaßnahmen und -entscheidungen nach Bildungsstatus, Zuwanderungszeitpunkt und Aufenthaltsdauer im Asylland unterscheidet.

„Nur was die Erziehung der Kinder betrifft, da finde ich trägt die Mutter mehr Verantwortung. Denn die Mutter trägt das Kind 9 Monate lang in ihrem Bauch, gebärt es und stillt es. Deshalb hat eine Mutter auch eine viel nähere Bindung zu ihren Kindern. “ (I64, männlich, seit 1983 in Ö.)

„Also, ich finde sowohl Frau als Mann sollten arbeiten gehen. ... Aber Kinder, ich würde schon sagen, ich find's besser wenn, natürlich die Frau die ersten paar Jahre daheim bleibt und auf die Kinder aufpasst. " (I45, weiblich, seit 1992 in Ö.)

\subsubsection{Karenz}

Auch die Karenz ist Teil des weiblichen Verantwortungsbereichs. Die Bereitschaft in Karenz zu gehen, ist unter in Österreich länger aufhältigen männlichen Befragten größer und ist mit der Voraussetzung eines höheren Einkommens der Frau verknüpft.

„Als meine Kleine geboren wurde, war ich noch in der Schule und da ist er in Karenz gegangen und hat auf die Kleine aufgepasst. Und ich habe die Schule dann beendet. Und wir machen das alles gleichzeitig. " (I32, weiblich, seit 1995 in Ö.)

„Das hat mir aber viel Erfahrung gebracht, weil ich dann zwei Jahre lang Hausmann war und habe mich um mein Kind gekümmert, während meine Frau weiterstudiert hat. “ (I30, männlich, seit 1984 in Ö.)

\subsubsection{Geschlechtsspezifisches Integrationsverhalten}

Folgende Zitate sollen abschließend einen prägnanten narrativen Einblick in die geschlechterspezifischen Orientierungen und die damit zusammenhängenden Herausforderungen im Verlauf individueller Integrationsprozesse bieten. Diese decken die unterschiedlichsten Lebensbereiche und Integrationsfelder ab, wie z.B. die eheliche und familiäre Rollenverteilung, das sukzessive Hineinwachsen in die Norm der, gender equality", die Rolle des Zeitfaktors im Rahmen der Internalisierung westlicher Rollenbilder, die generationenspezifisch divergierenden Schwierigkeiten hinsichtlich des Wandels von Rollenbildern, weibliche Erwerbstätigkeit als wichtige Determinante des Empowerment usw. 
„Für eine Frau, wenn sie ... sagen wir in einem System aufwächst, wo die einzige Person, die sie beschützen kann, in erster Linie ihr Vater ist, danach vielleicht ihr Bruder oder ein männliches Mitglied, weil nur er draußen gehen und kämpfen kann. Als Frau, wenn man rausgeht, ist man gar nicht sicher. Natürlich hängt man dann an dieser Kultur, dieser Entwicklung dann fest und man sagt: ,Mann gibt vor, und wenn er sagt das ist die Religion und da musst du gehen!' und so weiter. Und dann kommt man in ein anderes Land, wo man sagt: ,Ok, da brauche ich ja keinen Vater, keinen Bruder, da ist der Staat, da ist ein Gesetz' ..., da kann sich vieles ändern. Und ich sehe zum Beispiel bei vielen Frauen, die nach Europa kommen und Anerkennung und Status bekommen und dann auf einmal sagen, dass sie in einer Ehe jahrelang gelitten haben und dass das furchtbar war. Die sich für eine Scheidung entscheiden und gegen eine Ehe. " (E8)

„Sobald sie in Europa sind, dann merken sie: ,Aha, ich bin auch ein Mensch, mit jedem Recht das ein anderer hat, mit jeder Verpflichtung. 'Verpflichtung haben die Frauen immer in Afghanistan, aber die Rechte haben sie nicht. ... und wenn sie hierher [nach Österreich] kommen, dann haben sie keine Scheu bei McDonalds als Putzfrau zu arbeiten. Das ist ein Luxus für sie, das ist ganz normal. Sie verdienen ... zum ersten Mal in ihrem Leben ein selbständiges Geld auf ihrem eigenen Konto. Die Männer machen das nicht, haben es schwer. Die Männer, die dort eine Arbeit hatten, die ältere Generation, sagen wir mal über 30, haben es schwer, weil sie dort immer der Befehlshaber waren sozusagen, Befehlsgeber. Und jetzt haben sie es schwer, jetzt befehlen sie nicht mehr, wenn sie auf der Straße als Straßenkehrer arbeiten. Für die Frau ist es kein Problem, weil sie immer Befehlsempfängerin war. Und daher ist das [die gewonnenen Rechte] eine große Errungenschaft für die Frau. “ (E3)

„,... die Afghaninnen, die zu mir kommen, ja, ... die jungen Mädchen wollen alle lernen und wollen das machen und denen macht es Spaß in die Schule zu gehen, auch wenn sie zum ersten Mal in die Schule gehen, ja. " (E5)

„Generell kann man sagen, dass ... bei afghanischen Familien die Männer dominieren und das beobachte ich auch manchmal, wenn ein Ehepaar gemeinsam zu uns in die Beratung kommt und sitzt. Da sieht man, dass der Mann alles spricht. Manchmal sage ich ganz bewusst: ,Lassen Sie mal Ihre Frau sagen, was sie haben möchte [lacht] oder ob sie damit einverstanden ist hier zu unterschreiben oder etwas zu machen!"“(E6)

„Aber es gibt schon die Männer, vor allem die neu hier sind. Die denken schon, sie sind oben und Frauen sind unten. Wir zum Beispiel, wir sind nicht so. Weil wir sind schon lange hier in Wien. Überhaupt, die Leute, die schon lange hier sind, die denken nicht mehr so. Die sehen keinen Unterschied zwischen Mann und Frau. “ (I7, weiblich, seit 2001 in Ö.)

„Ich bin gebildet, ... ich interessiere mich eigentlich nicht so dafür die Arbeit zuhause zu machen [lacht]. Und meine Frau beschwert sich meistens. ... am 
meisten sollen die Frauen die Arbeit zuhause machen. Die Arbeit zuhause, da meine ich nicht, dass du für so etwas Geld kriegst: Kinderaufpassen, Kochen, Waschen, so was. Und die Männer müssen draußen arbeiten und Geld verdienen um die Familie zu unterstützen. " (I1, männlich, seit 2013 in Ö.)

„Ja, das hat sich geändert und wird sich weiter ändern. Zum Beispiel das sehe ich bei meiner Gattin, Als wir geheiratet haben, sie hat eigentlich total ein anderes Verhalten gehabt gegenüber mir und gegenüber den anderen Männern. Aber inzwischen besteht sie auf ihrem Recht und vor allem sie akzeptiert nicht, dass viel Blödsinn über Frauen erzählt wird. Sie ist [selbst]bewusster geworden. Sie vertritt ihre freie Meinung und vor allem sie versteht das. " (I37, männlich, seit 1988 in Ö.)

„Die Schwierigkeiten betreffen besonders mich und meinen Mann. Weil meine Mutter war eine alte Frau, natürlich das war sehr schwierig für meine Mutter, weil damals war ich 39 Jahre alt, für mich war es OK, für meinen Mann auch, für die Kinder war es am besten, aber für meine Mutter war es sehr schwierig. ... Aber ich meine, wir haben einen Spruch: ,Ich war so wie ... zwischen zwei Steinen - ich und mein Mann. 'Eine Seite waren meine Kinder, andere Seite war meine Mutter. Wir waren dazwischen. Auch mit unserem kulturellen Problem, weil die Jugendlichen ganz anders denken und meine Mutter ganz anders. Wir waren dazwischen - kaputt wirklich. Das war unser ... Problem damals. “ (I23, weiblich, seit 1988 in Ö.)

\subsubsection{Die Rolle der afghanischen Community}

Die Community in Österreich, wie auch die im Herkunftsland ansässige „extended family" nehmen erheblichen Einfluss auf den geschlechterspezifischen Verhaltenskodex. Unterschiedliche kulturelle und religiöse Vorstellungen der Generationen beinhalten ein mitunter erhebliches Konfliktpotential und setzen vor allem junge Frauen unter Druck, dem Idealbild einer Afghanin zu entsprechen.

„Ich war schon immer dafür, dass die Frauen die gleichen Chancen haben wie die Männer. ... In Österreich hatte ich halt mehr die Möglichkeit, mich darüber offen und kritisch auch demgegenüber zu äußern, warum Männer und Frauen nicht gleichgestellt sind. Und das versuche ich auch zu vermitteln - in der Community. ... Und wenn wir wirklich wollen, dass die Community oder die Gesellschaft vorangetrieben wird, dann müssen die Frauen emanzipiert sein, dann müssen sie gebildet sein. " (I9, männlich, seit 2007 in Ö.)

„Ich versuche hier auch feministisch zu sein, und zu sagen ... ,Du glaubst, dass du als Mann mehr Wert bist als ich, fertig!'. Das versuche ich zu erklären, aber sie wollen es auch akzeptieren, dass ich nachts rausgehe, aber es ist schwer für die, wenn mich wer sieht, und dann kommt er und sagt: ,Ach, ich habe die Tochter von dem Herrn ... gesehen, um zwei in der Nacht draußen. '... Weil sie so einen Druck 
haben, dass sie perfekt ausschauen für die anderen Menschen, für die anderen afghanischen Frauen oder Familien, dass sie alles in sich hineinfressen und alles perfekt darstellen möchten, dass sie irgendwie ... teilweise daran zerbrechen, weil sie nicht ehrlich sein können. " (I14, weiblich, seit 2001 in Ö.)

\subsubsection{Familiäre Erwartungshaltungen}

Vor allem junge Afghaninnen berichten von divergierenden Erwartungshaltungen gegenüber Mädchen und Burschen innerhalb der Kernfamilie und Community. Eltern tolerieren „liberales“ Handeln der Kinder eher, wenn diese männlichen Geschlechts sind. Mädchen und junge Frauen werden auch in Österreich verstärkt in häusliche Tätigkeiten eingebunden. Abhängig von Bildungsstatus und Zuwanderungszeitpunkt der Eltern wird der Fokus verstärkt auf die schulische Ausbildung beider Geschlechter gesetzt. Eine Mehrheit der weiblichen Befragten distanziert sich von traditionell orientierten Erwartungshaltungen der älteren Generation.

„B1: Von klein auf Kochen lernen und putzen und so. B2: Wie ich! Ich warglaube ich-fünf Jahre alt, als ich meiner Mutter geholfen habe. Vier Jahre als ich mit meiner Mutter angefangen habe zu kochen, zu putzen. ... I: Und deine Brüder nicht? B2: Nein! Die können zwar arbeiten, aber die wollen nicht. " (I6, weiblich, seit 2003 in Ö.; I7, weiblich, seit 2001 in Ö.)

„,Und mein Vater glaubt nicht an sowas. Er sagt immer, er versucht seine Kinder gleich zu behandeln. Jeder sollte unabhängig sein, es ist wurscht, ob du jetzt ein Junge oder ein Mädchen bist. Man sollte dazu fähig sein, wenn man eines Tages auf sich allein gestellt ist, dass man selbst dazu fähig ist, sein eigenes Leben zu führen. " (I10, weiblich, seit 2006 in Ö.)

Die Realisierung gleichberechtigter Rollenkonzepte variiert je nach Herkunftsgebiet der afghanischen Familie. So orientieren sich ländlich geprägte Geschlechterrollen im Vergleich zu urbanen Rollenbildern vermehrt am traditionellen Wertesystem Afghanistans und die Erwartungshaltungen gegenüber den Geschlechtern unterscheiden sich beträchtlich.

„Für mich und für meinen Mann war meine Tochter - wie sagt man das? Oberschicht von meinen zwei Söhnen [den Söhnen gegenüber höhergestellt]. Wenn wir etwas gekauft haben, dann zuerst für meine Tochter, dann für meine zwei Söhne. ... manche Familien, ... wenn sie ausgebildet [gebildet] sind, sie verstehen das, aber [wenn sie nicht gebildet sind] sie haben eine andere Idee. Sie sagen: ,Okay, Söhne gehören zu uns. Aber Tochter ist nicht von uns. Weil wenn sie heiraten, sie [Töchter] gehen weg von unserem Zuhause. 'Deshalb respektieren sie die Mädchen, manche Familien. ,Okay, ein paar Jahre ist sie bei uns, dann wird verheiratet. Sie kriegt eigene Probleme, mit anderer Familie, mit anderen Beziehungen, deshalb müssen wir unserer Mädchen respektieren. 'Aber manche Familien, nein, das geht nicht. Mädchen ist Mädchen, Bub ist Bub, so ist das. “ (I23, weiblich, seit 1988 in Ö.) 


\subsubsection{Der Generationenkonflikt im Spannungsfeld zwischen zwei Kulturen}

Gegensätzliche Einstellungen zu Geschlechterrollen und -beziehungen zwischen älterer und jüngerer Generation sind auf divergierende kulturelle Normen zurückzuführen, die in einem interkulturellen Austausch ein erhebliches Konfliktpotential in sich tragen. Aufgrund der raschen strukturellen (Schulpflicht) und sozialen Integration von jungen Afghan/inn/en und - wie eine Expertin im Integrationsbereich beschreibt - der größeren „Offenheit“, neue Sozialstrukturen anzunehmen, sind Emanzipationsbestrebungen hinsichtlich gleichberechtigter Geschlechterbeziehungen unter jüngeren Befragten vorherrschend.

„... man ist einerseits Afghane, aber man lebt in Österreich. Man versucht das Beste aus beiden mitzunehmen oder zu mischen. Und das fällt nicht einfach. Ich meine, meine Mama ist noch jung. Ich versuche ihr alles beizubringen oder das, was ich lerne oder was ich kenne. Aber nicht jede Mama ist so jung wie meine. Die anderen sind alle 50. Wie willst du eine 50-jährige Frau, die nicht zur Schule gegangen ist, [erziehen]? “ (I14, weiblich, seit 2001 in Ö.)

„I: Wie ist das bei deinen Eltern? B: Mein Vater war am Anfang z.B. nicht der gleichen Meinung wie ich. Er hat gemeint: ,Nein, man muss Frauen halt beschützen. Sie sollten nicht zu lange draußen bleiben. Was ist, wenn ihnen was zustößt und so weiter. 'Also er denkt wahrscheinlich, dass sich Frauen selbst nicht wehren können. Aber die letzten drei Jahre ... also ich diskutiere mit ihm immer wieder sehr häufig über Politik, über Frauenrechte und so weiter. Und mittlerweile sieht er das ein. Dass Frauen genauso die gleichen Rechte haben sollten wie die Männer. ... Meine Mutter tut sich noch immer schwer. Sie denkt immer noch so, wie sie selbst erzogen worden ist. Dass halt die Töchter Kopftuch tragen sollten und nicht zu spät nachhause kommen sollten und sich gut benehmen sollten. Und zuhause viel arbeiten und so weiter. " (I9, männlich, seit 2007 in Ö.)

\subsubsection{Interaktionen mit der österreichischen Mehrheitsgesellschaft als Determinante von Einstellungsänderungen}

Im Rahmen politisch gesetzter Integrationsmaßnahmen, wie der Werte- und Orientierungskurse, wird den Teilnehmenden die rechtliche und soziale Gleichstellung von Mann und Frau in Österreich vermittelt. Dennoch bestätigt ein Experte eine anfängliche Überforderung bei vielen der kürzlich eingereisten Afghan/inn/en. Der soziale Austausch mit der österreichischen Mehrheitsgesellschaft und ein damit verbundener Zeitfaktor sind ausschlaggebende Faktoren, um einem anfänglich verunsicherten Agieren gegenüber dem anderen Geschlecht entgegenzuwirken und die Gleichstellung zu internalisieren.

„,... interessiert, teilweise schwer verunsichert. Teilweise große Verunsicherung in der Frage: ,Wie kommuniziert man? Wie funktioniert Flirten zum Beispiel?'

... Oder auch Verunsicherung von den Burschen her: ,Was ist das, wenn eine 
Frau sehr selbstbewusst und offensiv und selbständig ist?' So mit Rollensituationen und gleichzeitig auch totale Wissbegierde zu lernen. Also ich glaube, grad bei den Jungen ist das nur eine Frage der Zeit, bis sich das einpendelt irgendwie ... Kontakt zu Österreichern, zu Österreicherinnen, einfach ein Lernprozess. Wie tickt die Gesellschaft? Das ist ja nicht so einfach. ... Und wenn du frisch von ganz woanders kommst, muss das ja teilweise ein totaler Kulturschock sein. Das lernen, das einordnen zu können. Wo sind Prioritäten? Was ist wirklich ernst? Welche Konsequenzen? Was wird von mir wirklich auch erwartet? Ich stelle mir das wahnsinnig schwer vor. "(E10)

\subsection{4 (Ehe-)Partner/innenwahl}

Familiärer Einfluss bei der (Ehe-)Partner/innenwahl, welcher auf ein traditionell verankertes Wertesystem zurückgreift, ist auch im Asylland allgegenwärtig. Obwohl die österreichische Rechtslage auch Frauen befähigt, einen Scheidungsantrag zu stellen und junge Befragte der Auflösung der Ehe weniger kritisch gegenüberstehen, üben bestimmte Erwartungshaltungen der Eltern und der „extended family“ Einfluss auf einschneidende Lebensentscheidungen der jüngeren Generation aus. Dies trifft auch auf die (Ehe-)Partner/innenwahl zu. Einer Präferenz zur selbständigen Partner/innensuche seitens zahlreicher junger Befragter kann somit aufgrund eines moralisch determinierten Verhaltenskodex nicht immer entsprochen werden.

„Ich war davor jemand, der mit den Klassenbesten konkurriert hat, aber als ich verlobt wurde, war all das nicht mehr in meinem Kopf. Ich habe mir gedacht, egal wie hart du lernst, von morgens bis abends lernst, wirst du zum Schluss in eine andere Familie gehen ... Dann habe ich eben geheiratet. “ (I61, weiblich, seit 2014 in Ö.)

„In Afghanistan entscheiden eigentlich die Eltern für die Kinder, wen sie heiraten sollen. Denn sie dürfen nicht einen Freund haben. ... Sie sagen: ,Ich suche einen Bräutigam für meine Tochter. '... Zum Beispiel X ist meine Cousine. Ich habe sie geheiratet. Und wenn ich das Leuten hier erkläre, sind sie eigentlich geschockt, dass ich meine Cousine ... wegen dem Kind, ob es gesund wird oder nicht. ... So war das am Anfang schwer. Kompromiss, Kompromiss, bis wir jetzt zufrieden sind. " (I1, männlich, seit 2013 in Ö.)

\subsubsection{Die Wichtigkeit religiöser und ethnischer Zugehörigkeit}

Eltern bevorzugen für ihre Kinder Partner/innen, die aus derselben religiösen und ethnischen Gruppe stammen. Dabei wird der religiösen Zugehörigkeit größere Bedeutung zugeschrieben als der ethnischen. Also Ehen mit Muslim/inn/en sind generell wichtiger als eheliche Verbindungen mit Afghan/inn/en. Je nach der Aufenthaltsdauer in Österreich und der Religiosität variieren die Präferenzen der Eltern. Ist es Kindern gestattet, ihre Partner/innenwahl selbst zu treffen, dann ist die Toleranz der Eltern gegenüber 
nichtmuslimischen Partner/inne/n höher. Personen aus einem traditionell orientierten afghanischen Kontext tendieren dazu, sich mit Partner/inne/n derselben ethnischen und religiösen Zugehörigkeit zu liieren.

„I: Wäre das dann wichtig für dich, dass er auch Muslim ist? B: Ja, ganz, ganz wichtig ist für mich. I: Und auch, dass er Afghane ist oder wäre das egal? B: Nein, ist mir egal, ob er Afghane ist oder von einem anderen Land. " (I44, weiblich, seit 2015 in Ö.)

„I: Und war es für deinen Bruder wichtig eine Afghanin zu heiraten? B: Ja, auf jeden Fall. I: Wäre es wichtig für dich? B: Für mich wäre es nicht wichtig. Für meine Eltern wäre es sehr wichtig. " (I12, weiblich, seit 2006 in Ö.)

Einige Respondent/inn/en befürworten eine „kulturelle“ Ähnlichkeit und argumentieren dahingehend, dass die Ehen von Personen mit kulturellen Ähnlichkeiten dauerhafter seien und sich glücklicher gestalten.

„Aber mir wäre schon - muss ich sagen - Muslim wäre mir lieber. Weil allein schon bei der Kindererziehung, später dann ist man sich einig drüber, wie man das Kind erzieht oder in vieler Hinsicht hat man dann vielleicht oft mehr Gemeinsamkeiten. “(I45, weiblich, seit 1992 in Ö.)

„Ja, muss Muslim sein! Wenn man diesen Menschen heiratet, das heißt miteinander zurechtkommen. Aber wenn er Muslim ist, ist es besser. ... I: Okay, der Mann für Ihre Tochter sollte Muslim sein oder für beide? B: Ja, er muss Muslim sein, weil ich möchte nicht, dass die Kultur, die wir haben, verschwindet. " (I58, weiblich, seit 2016 in Ö.)

\subsubsection{Die Wahl österreichischer Partner/innen}

Ein gewisses Ausmaß an pragmatischem Handeln ist auch bei der Partner/innensuche vorhanden. Einige Befragte sehen einen erheblichen Vorteil darin, sich mit Personen aus der österreichischen Mehrheitsgesellschaft zu liieren und erwähnen einen damit assoziierten strukturell-integrativen Nutzen (Wohnen, Sprache, Arbeitsplatz etc.). Des Weiteren ist zu vermerken, dass asyl- und schutzberechtigte Afghan/inn/en mit einem/r österreichischen Partner/in viel eher dazu neigen, traditionell geprägte Rollenbilder, die in Afghanistan vorherrschend sind, abzulegen.

„Nein, ich werde nicht eine Afghanin heiraten, sondern eine von hier - aus Europa. Erstens möchte ich mein Leben aufbauen und hier ist es leichter. Wir, die hier leben, nehmen langsam, Schritt für Schritt, ihre Tradition und Werte an. Es gibt einen Spruch: ,Wenn das Leben nicht mit mir zurechtkommt, dann musst du mit dem Leben zurechtkommen. 'Ich möchte mit ihrer Tradition und Kultur zurechtkommen. Ich würde gerne eine Österreicherin heiraten. " (I56, männlich, seit 2015 in Ö.)

„Nein, meine Tochter ist verheiratet mit einem Österreicher. Er ist auch Arzt, praktischer Arzt. Sie haben sich an der Uni kennengelernt. Und obwohl es von 
unserer Seite, von unserer afghanischen Seite, ein Tabu war und jeder gesagt hat: ,Tochter von XY hat geheiratet Österreicher ... ““ (I23, weiblich, seit 1988 in Ö.)

\subsubsection{Suchen und Finden - Unterschiede zwischen den Geschlechtern}

Eine Mehrheit der Respondent/inn/en erwähnt den Einfluss der Eltern bei der Wahl potentieller (Ehe-)Partner/innen, die allerdings je nach Geschlecht der Kinder differiert. Die Toleranz der Eltern gegenüber Heiraten außerhalb der religiösen und ethnischen Gruppe ist bei Söhnen höher als bei Töchtern:

„Umgekehrt ist es ein bisschen schwieriger, weil ein muslimischer Mann kann auch andersgläubige Frauen heiraten, weil die ja patrilinear denken und die Kinder somit nicht dem Islam entzogen sind. Aber wenn die Mutter Muslima ist und der Vater ..., dann ist ja deren Logik, dass die Kinder dann für den Islam verloren sind, weil es ja nach dem Vater geht. “(E1)

„,Ich habe kein Problem damit, wenn mein Sohn ein Mädchen heiratet, das nicht aus unserem Land kommt bzw. keine Muslimin ist. Bei meiner Tochter bin jedoch strenger. Bei ihr würde ich dies nicht erlauben. ... Weil wenn mein Sohn eine Nichtmuslimin heiratet, kann er sie in unsere Kultur und unsere Religion ein wenig einweihen, aber meiner Tochter würde ich dies nicht erlauben. " (I57, weiblich, seit 2015 in Ö.)

„, Meine Mutter hat gesagt, also du musst heiraten ... Irgendwie sie war sehr arm, also ich konnte Sie nicht sehen. Also sie hat geweint, das war ihr letzter Wunsch. ... Und irgendwie hab 'ich ... Mama war sehr alt und dann habe ich geheiratet und bin nach Österreich ... ca. nach 6 Monaten. “ (I22, männlich, seit 1986 in Ö.)

Auch fühlen sich Töchter eher dazu verpflichtet, den elterlichen Partnerwahlpräferenzen entsprechend zu agieren. Ein „Nein“ der Kinder zu einer/m bevorzugten Partner/ in der Eltern wird zwar in der Regel akzeptiert, die elterliche Erwartungshaltung und der Druck seitens der afghanischen Familie und Community die „richtige“ Wahl zu treffen, drängen die Kinder jedoch häufig dazu, der elterlichen Präferenz zu entsprechen.

„,Mir haben sie deshalb gesagt ich soll ihn heiraten, weil er aus unserer näheren Familie ist. Als sie um meine Hand anhalten gekommen sind, haben sie mir gesagt: , Weil er aus unserer näheren Familie ist, können wir nicht nein sagen. ' Er ist mein Cousin und sie haben gesagt, dass ich ihn heiraten soll. Sie haben oft um meine Hand angehalten, aber ich habe immer abgelehnt. Und dann musste ich .... " (I61, weiblich, seit 2014 in Ö.)

„, Aber es ist total okay, wenn ein afghanischer Junge eine andere Frau, also eine Nichtafghanin heiratet. Aber es kommt gar nicht in Frage, wenn es andersrum ist. Das ist wirklich, wirklich nervig. ... z.B. habe ich eine Freundin, ihr Bruder hat eine Österreicherin geheiratet, und es war bei ihm überhaupt kein Problem. Oder der Vorstandsvorsitzende von X, ein Freund von mir, der hat eine Mongolin 
geheiratet und es ist überhaupt kein Problem. Aber wenn es ein Mädchen machen würde, da würden alle aufschreien! " (I12, weiblich, seit 2006 in Ö.)

\subsubsection{Wenn die Liebe siegt!}

Einige Eltern befürworten die eigenständige Partner/innenwahl der Kinder und lehnen eine traditionell orientierte afghanische Heiratsvermittlung ab. Gründe dafür sind u.a. eine bessere und länger währende Ehe aufgrund des davor zeitlich begründeten besseren Kennenlernens des Paares und eine Befürwortung der persönlichen Entscheidungs- und Handlungskompetenz. Der Druck seitens der afghanischen Community aufgrund deren dichter Vernetzung und einflussreicher familiärer Entscheidungsmacht schränkt jedoch die Toleranz der Eltern gegenüber einer freien Partner/innenwahl ein. So sind Kinder, deren Partner/innenwahl nicht zur elterlichen Zufriedenheit erfolgt, manchmal dazu geneigt, sich von Familie und Community bewusst abzugrenzen.

„,Also meine Freundin ist nicht Afghanin und sie ist auch keine praktizierende Muslimin und das wissen auch meine Eltern. Am Anfang war es schwierig. Aber ja, ich habe gesagt, das ist mein Leben, so möchte ich es leben und das ist für sie jetzt in Ordnung - auch für meine Geschwister. Meine Schwester lebt nicht zuhause, sie lebt alleine, und auch das ist für sie in Ordnung. Also ich bin froh, dass meine Eltern jetzt so weit gekommen sind. Aber trotzdem muss noch vieles passieren. Ich darf jetzt nicht nachgeben. Also bis sich mein Vater wirklich auf die Seite der Frauen gestellt hat und sagt: ,Jetzt bin ich hundertprozentig davon überzeugt und ich unterstütze euch. "“ (I9, männlich, seit 2007 in Ö.)

„B: Also wie gesagt, ich habe mit meinen Eltern viel gesprochen, viel gestritten, viel diskutiert über solche Sachen und die merken, also die wissen, dass X und ich total anders sind, einfach als die anderen afghanischen Mädchen, die hier groß geworden sind. Meine Schwester hat einen Freund. Und meine Eltern haben das herausgefunden. ... sie wissen, dass meine Schwester keine Jungfrau mehr ist. Und ich meine, es war alles natürlich schlimm für sie ... Aber sie kommen damit klar, sie versuchen damit klar zu kommen. ... Aber meine Cousine hat einen Franzosen geheiratet, und ihre Mama ist auch megastreng und konservativ, aber langsam bürgert sich das so ein bei den Afghanen, dass wir auch, dass die Frauen noch mehr Mitspracherecht haben. Und da mein Papa ziemlich offen ist, was das angeht. Das Wichtigste für sie ist einfach, dass er eine Ausbildung hat, dass er kein Alkoholiker ist .... " (I14, weiblich, seit 2001 in Ö.)

\subsection{Diverse Einstellungen und Wertehaltungen}

\subsubsection{Empirischer Forschungsstand}

Es würde den Rahmen dieses Abschnitts sprengen, wollte man die kontroversielle Wertedebatte in zahlreichen wissenschaftlichen Disziplinen auch nur annähernd wieder- 
geben. Den empirischen Belegen sollen aber zumindest einige Begriffsbestimmungen vorangestellt werden.

Werte sind Aspekte einer sozial geteilten Konstruktion von Wirklichkeit, die sprachlich vermittelt - als Bewertungsgrundlagen von Handlungen innerhalb einer Gesellschaft zur Anwendung gelangen. Sie beziehen sich auf mehr oder weniger verbindlich Erstrebenswertes. Indem Individuen die gesellschaftlich konstituierten Werte interpretieren, wird der Person eine individuelle Werthaltung zugeschrieben, die ihre Handlungen mit jenen anderer vergleichbar macht. ${ }^{110}$ Die klassische Definition von Clyde KLucKhohn (1954) lautet: ,ein Wert ist eine explizite oder implizite, für das Individuum kennzeichnende oder für eine Gruppe charakteristische Konzeption des Wünschenswerten, die die Selektion von vorhandenen Arten, Mitteln und Zielen des Handelns beeinflusst“. Milton Rokeach (1973) definierte Werte als „eine relativ konstante Überzeugung, dass eine spezielle Art des Verhaltens oder ein existentieller Zielzustand dessen Gegenteil oder Umkehrung persönlich oder sozial vorzuziehen ist.“ Wolf-CsanÁrdy baut auf Kluckhohns Kulturtheorien auf und betont folgende Merkmale: 1) Die Orientierungs- und Selektionsfunktion von Werten; 2) Die Tatsache, dass der „Träger“ sich nicht unbedingt seiner Werte bewusst sein muss und 3) ihre Verankerung in sozialen Kollektiven (1996, p. 32 ff.).

Eine empirische Studie zu Wertehaltungen von Geflüchteten aus Afghanistan, dem Irak und Syrien in Österreich wurde 2017 vorgelegt. ${ }^{111}$ Aufschlussreiche Einblicke in die Einstellungen der 2015 nach Österreich Geflüchteten bieten auch die Analysen von Kohlenberger und Buber-Ennser (2017). BraAkman (2005) weist darauf hin, dass das Alter der Person ein wichtiger Faktor ist, um Einstellungen zur neuen Umgebung und im Immigrationskontext geltenden Wertsystemen zu verstehen. Bezüglich des traditionellen Wertesystems in Afghanistan sei auf EmADi (2005, p. 136 ff.) verwiesen, zum Wertekodex der Paschtunen vgl. KaKar (2003) und RzeHaK (2011).

\subsubsection{Analyse der Interviews}

\subsubsection{Gewalt}

Die Gewaltthematik stellt ein politisch besonders sensibles Thema dar. Ein Experte führt das (ausschließlich männlich assoziierte) gewaltorientierte Verhalten auf die bereits 40 Jahre andauernde Krise in Afghanistan zurück:

„Das heißt, wenn in einem Land seit 40 Jahren Krieg ist und eigentlich man nur Gewalt erlebt, was will man eigentlich von diesen jungen Leuten erwarten? Die dann herkommen, einige - ich meine, es sind nicht alle. ... Der Großteil ist in Ordnung. Diese Leute sind eigentlich sehr arm in meinen Augen. Sie brauchen Hilfe. Das, was in den Medien steht, ist leider Gottes sehr unangenehm, aber das

110 Vgl. dazu <www.uni-kassel.de/fb4/psychologie/personal/lantermann/sozial08/werte.pdf $>$.

111 Vgl. KoHLBACHER et al. 2017 <https://www.bmeia.gv.at/integration/download/publika tionen/>. 
macht ein normaler Mensch nicht. Und wenn jemand praktisch da so viel Gewalt erlebt hat, dann ist man irgendwann einmal verrückt. Manche halten das aus und manche nicht. " (E7)

Bereits seit Jahrzehnten in Österreich lebende Afghan/inn/en sehen die heutige Gewaltbereitschaft kritisch. Flüchtlinge der 1970er- und 1980er-Jahre konnten noch in einem stabilen Normensystem aufwachsen und hatten ihre Schulausbildung regulär absolviert, während die rezent Geflüchteten aus einem kaum funktionierenden staatlichen, gesellschaftlichen und wirtschaftlichen System stammen.

„Damals waren die Familien keine Kriegsflüchtlinge, die waren eigentlich aus ,normalen '- mit Anführungszeichen - Familien nach Europa ausgewandert, aber heute sind sehr viele Menschen darunter, die entweder Gewalt erlebt haben oder Gewalt verübt haben. " (I30, männlich, *1953, seit 1984 in Ö.)

Traumatische Erfahrungen spielen als Kausalfaktoren für Gewaltbereitschaft eine wesentliche Rolle. Allerdings wirken viele Einflussgrößen kumulativ auf das Gewaltpotential.

„Oder überhaupt, wenn man so Klientel aus Kriegsgebiet hat und wo doch so lange Zeit Lebensbedrohung ist, Gewalterfahrung, wie weit das einfach wirkt ...? Man kann nie eine Eins-zu-eins-Übersetzung machen und sagen: ,So und so viel Prozent nehmen sie aus Afghanistan mit. So und so viel Prozent ist der Stress, den sie hier als Asylwerber haben. So und so viel Stress haben sie dann bei der Integration, wenn sie bereits den Asylstatus haben.' Ich könnte Ihnen das jetzt nicht auseinanderhalten und sagen: ,Ja, ja, das ist der Grund, warum da jetzt der Stresspegel so hoch ist. Es ist kumulativ. "“(E9)

Gewalt ist auch ein Phänomen, welches in Bezug auf das Leben in den Asylquartieren erwähnt wird:

„, Wie in jeder Familie gibt's natürlich auch dort in den einzelnen Quartieren, a bedingt durch die räumliche Situation teilweise a Gewalttaten. Und dann ist eh pro Quartier ein Exekutivbeamter namhaft geworden für den Quartierbetreiber, an den man sich gleich wenden kann und dann kommt's natürlich auch zu Wegweisungen immer wieder. "(E10)

Das Problem der Gewalt von Vätern gegenüber Kindern wird mehrfach angesprochen. In Familien mit Gewaltproblematik wird auch die Ehefrau zum Opfer. Eine Expertin erwähnt auch die von Müttern ausgeübte Gewalt. Ein Risikofaktor manifestiert sich in dem für Afghanen ungewohnten Alkoholgenuss, der Aggressivität und innerfamiliäre Gewalt fördert, während die in Afghanistan gebräuchlichen, traditionellen Drogen eher zu Teilnahmslosigkeit führen.

„Die fangen dann zu Trinken an. Während Opium schläfrig macht, macht der Alkohol aber aggressiv und dann, clasht' es. Eine Bewältigungsstrategie, die man ja auch sehr gut kennt, und das betrifft dann eher Familien, dass dann die ohnehin 
schon latente Gewalt sich noch verstärkt. Also, dass immer der, der oben ist, tritt nach unten und dann die Mütter treten auf die Kinder. ... Was ganz interessant ist, da gibt es aber glaub 'ich keine statistischen Daten bisher, aber dass es doch nicht selten vorkommt, dass Afghaninnen, wenn sie dann da sind, die Scheidung einreichen. Also oft geht vorher Gewalt in der Familie einher und dann kommen die in Frauenhäuser und werden betreut und dann reichen sie die Scheidung ein. " (E1)

Die Vermittlung eines Bewusstseins für Kinder- und Frauenrechte sowie das Propagieren eines gewaltfreien Erziehungsstils wird von einem Experten als wichtiger Aspekt der Orientierungskurse angeführt. Deshalb werden Informationen zur gewaltfreien Erziehung auch in die Lerninhalte der Kurse eingeflochten.

„,Und jetzt hamma als zweiten zusätzlichen Schwerpunkt den Bereich Kinderrechte .... Da haben wir eben unterschiedliche Maßnahmen vorbereitet mit verschiedenen Institutionen, UNHCR, UNICEF, IOM ..., dass man die Familien, die da sind, stärkt und auch über die verschiedenen Rechte der Kinder aufklärt. ... dass man die Familie dementsprechend sensibilisiert auf das, was ist bei uns verboten bzw. was ist Pflicht, wie der Schulbesuch zum Beispiel. Wie schaut gewaltfreie Erziehung aus? Wie funktioniert das, dass Mädchen nicht diskriminiert werden?" (E10)

Eine afghanische Frau bewertet das empathische Verhalten der Lehrer/innen im österreichischen Schulsystem Schüler/inne/n gegenüber positiv und berichtet kontrastierend dazu über strukturelle Gewalt im afghanischen Bildungssystem.

„,Meine Kinder hat viel Recht hier. Sie können in Ruhe lernen und lernen mit Spaß. Das macht viel mehr Erfolg. ... Aber in Afghanistan immer die Lehrerinnen ... schlagen die Kinder mit dem Holz an Hand. ... Aber hier, Gott sei Dank, kein Schlagen gibt es. “ (I19, weiblich, *1973, seit 2011 in Ö.)

Die den männlichen Afghanen undifferenziert zugeschriebene Gewaltbereitschaft stellt eine Barriere für deren Arbeits- und Wohnungsmarktintegration dar. Bei Abschiebungen wird seitens der Familien auch häufig angenommen, die Rückkehrer hätten in Europa Gewaltdelikte verübt, was eine Wiederintegration in die Herkunftsfamilie erschwert.

„,... die Menschen haben alles ins Afghanistan verloren und ... keine Chance wieder dorthin zurückzukehren und dort ihr Leben aufzubauen, weil dort die Familie nicht mit der Angst und mit dem Gerede der anderen Menschen umgehen kann: ,Du warst in Europa, was hast du Schlimmes gemacht? Hast du jemanden vergewaltigt? Hast du gestohlen? Hast du Drogen verkauft, dass du dort [in Europa] nicht geblieben bist und wieder nach Afghanistan abgeschoben wurdest? "“(E12)

Eine spezielle Problematik manifestiert sich in kulturellen Missverständnissen zwischen afghanischer und österreichischer Seite im sensiblen Feld sexuell konnotierter Übergriffe. Durch divergierende kulturelle Normen geleitetes Verhalten kann zu Fehlin- 
terpretationen auf beiden Seiten führen. Rigide traditionelle Moralvorstellungen und Geschlechtersegregation (vgl. RzEHAK 2011) kollidieren mit den Möglichkeiten des Internet, was dazu führt, dass westliche Frauen vor dem Hintergrund der moralischen Werte afghanischer Männer falsch eingeschätzt werden.

„,... er hat nicht verstanden, ja die Frau war nett. Bei ihm im Land hat er das nicht gesehen, dass eine Frau mit ihm lacht. Wenn eine Frau mit einem Mann lacht, dann denkt er automatisch, tut mir leid, dass ich das so sage, ... Muss man sofort versuchen mit ihr Sex zu haben. ... das braucht Zeit, das braucht Zeit am Anfang. " (E11)

\subsubsection{Materielle Werte}

Die Erwirtschaftung eines eigenen Einkommens zählt zu den durchgängig verbalisierten Lebenszielen und genießt einen hohen Stellenwert. Ein wichtiger Teilaspekt finanzieller Transaktionen, der auch zu einem eigenen Einkommen in einem Konnex steht, ist jener der „remittances“ an Familienangehörige im Herkunftsland. In der Erwartung Überweisungen zu erhalten, manifestiert sich auch eine wesentliche Determinante des Drucks zum raschen Einkommenserwerb.

„Ich glaube, die sind auch unter dem Druck, dass sie entweder Geld nachhause schicken und weil sie oft eben nicht im Familienverband herkommen, müssen sie um Familienzusammenführung, die ja schon schwierig ist, überhaupt zu ermöglichen, brauchen sie ein Einkommen, ein geregeltes. “ (E1)

„,... die war meine erste Frau, sie ist geblieben in Afghanistan. ... Natürlich, Geld schicke ich immer, noch immer schicke ich. Und meinen Kindern auch schicken Geld. “(I33, männlich, *1946, seit 1974 in Ö.)

Eine gesellschaftliche Schande wäre es, nach Afghanistan zurückzukehren und über keine finanziellen Mittel zu verfügen. Die Geflüchteten befinden sich also vielfach in einem Dilemma, denn einige würden aufgrund ihrer Erfahrungen in Österreich sogar die Rückkehr erwägen, können diese aber aus Furcht vor Stigmatisierung infolge ihrer Mittellosigkeit nicht antreten.

„,... und da hier eine Existenz zu gründen. ... Weil das wäre dann dort als Versager sozusagen, wenn du zurückkommst. " (I38, männlich, *1951, seit 1974 in Ö.)

\subsubsection{Sicherheit}

Der Wert der persönlichen, gesellschaftlichen und politischen Sicherheit wird in unterschiedlichsten Zusammenhängen in vielen Interviews erwähnt. Bei der Bewertung Österreichs wird sie als ein Positivfaktor hervorgehoben und der allgemeinen Unsicherheit in Afghanistan gegenübergestellt. Diese Unsicherheit hat die Alltags- und Lebensgestaltung nachhaltig geprägt.

„,... die Sicherheit ist gut hier. Was die Sicherheit angeht, muss man sich keine Sorge machen. Deine Kindern können sich ruhig um ihre Aufgaben kümmern. Was 
das angeht, hier ist es besser im Vergleich zu Afghanistan. “ (I58, weiblich, *1986, seit 2016 in Ö.)

„, Wegen der Sicherheit, kann ich einfach Beispiel am Abend, in der Früh, egal wann, kann ich von Haus weggehen. " (I24, weiblich, *1996, seit 2015 in Ö.)

„,Ich hatte keine Information über Österreich, oder andere Länder. Ich wollte nur in Sicherheit sein, weil mein Leben in Gefahr war, deswegen habe ich mein Land verlassen. " (I60, weiblich, *1960, seit 2006 in Ö.)

Als besonders wichtig wird die in Österreich vorhandene Sicherheit von Geflüchteten mit Kindern bewertet. Im Zusammenhang mit der Sorge um die Zukunft der Kinder spielt der Sicherheitsfaktor auch bei der Abwägung der Möglichkeiten einer etwaigen Rückkehr eine wichtige Rolle. Denn Remigration wird vor allem wegen der Kinder und aus Gründen der Sicherheit ausgeschlossen.

„,... und bin ich auch alleine mit dem Kleinen, einjähriges Kind, deswegen bin ich nach Österreich gekommen, dann ich wollte nicht mehr weitergehen. Ich wollte gerne hierbleiben. Zwei bis drei Tage habe ich schon überlegt, dann habe ich gesagt: ,Nein, es passt für mich, es ist sehr schön, Sicherheit."“ (I35, weiblich, *1974, seit 2004 in Ö.)

\subsubsection{Persönliche Freiheit}

Persönliche Freiheiten werden in westlich-säkularen Gesellschaften anders bewertet als in der afghanischen (vgl. EMADI 2005, RzEHAK 2011). Es bestehen also Missverständnisse in Bezug auf die Einhaltung von Terminen und generell des Ausmaßes an persönlichen Freiräumen. In den Herkunftsgesellschaften dominieren über TV und Internet vermittelte klischeehafte Vorstellungen „absoluter Freiheit“ in westlichen Gesellschaften, welche mit der Realität in Kollision geraten. Die Internalisierung des Wertes persönlicher Freiheit, der sich an vorgegebenen Grenzen orientiert, ist individuell unterschiedlich und kann einige Zeit in Anspruch nehmen.

„Ja, sagen wir, Sie sagen einer Person: ,Jetzt sind Sie in Österreich frei. 'Wie frei bin ich dann? Darfich auf der Straße herumlaufen? Darf ich mir nehmen, was ich will? Darf ich jede Frau ansprechen? ... ,Ok, in Europa, sie können machen was sie wollen. 'Ja, das wurde den Menschen gesagt. Oder: ,Die Menschen machen dort was sie wollen, was ihnen gefällt.' Na, können sie wirklich machen, was ihnen gefällt? Das heißt sie können morgen nicht zu ihrem AMS-Termin gehen und sagen: ,Ich komme nicht, einfach weil's mir nicht gefällt. 'Geht nicht. Oder: ,Ich schlafe heute bis zwölf Uhr, weil es mir gefällt. ' ... dass wir einfach viele Menschen haben, die diese Systeme gar nicht kennen, ... Müssen wir sehr weit zurückgehen auf ganz einfache Fragen, so verständlich denen zu sagen ... Natürlich, wenn sie dann sagen: „Ah, es ist eine Demokratie, aber ich darf noch immer nicht! Also ich muss halt meinen AMS-Termin jetzt besuchen und ich muss jetzt um neun Uhr aufstehen, dann gefällt mir dieses System schon gar nicht. “ (E8) 
Missverständnisse bezüglich des individuellen Entscheidungsspielraums treten auch hinsichtlich geforderter Regelmäßigkeit der Partizipation etwa an Deutschkursen oder beim Schulbesuch auf.

„,Vor allem dachte ich mir, wenn man in Österreich ist, kann man sich selbst aussuchen, ob man in der Schule sitzen möchte oder nachhause gehen möchte. Man darfjederzeit rein und raus. ... ich dachte, man könne jeden Tag in die Schule gehen, falls man mag. " (I10, weiblich, *1997, seit 2006 in Ö.)

Einen speziellen Aspekt repräsentiert die Pünktlichkeit in der Einhaltung von Terminen, der Fertigstellung von Aufgaben etc. Die konsequente Inkorporation ins eigene Leben stellt für manche Befragte eine erhebliche Herausforderung dar.

„Ich hab' einfach versucht, pünktlich zu schlafen, pünktlich aufzustehen, pünktlich in die Arbeit gehen, pünktlich nach Hause kommen, meine Wäsche waschen, kochen, vor Abend, in der Früh joggen gehen, oder Radfahren gehen. Also Beschäftigung hat mich komplett geändert und da bin ich auch froh. " (I27, männlich, *1991, seit 2005 in Ö.)

\subsubsection{Individueller Wertewandel}

Der Wandel des persönlichen Wertesystems findet zwar statt, wird aber nur von wenigen Interviewten beschrieben. In einem Fall etwa hat eine grundlegende Veränderung in Richtung eines verantwortungsbewussten Lebens stattgefunden. Hinsichtlich des Wertewandels besteht ein enger Konnex zur Aufnahme einer regelmäßigen Beschäftigung.

„,... Seit 2008 hat mein Leben sich viel geändert. ... Und jetzt, da ich in Sozialberuf bin, meine Einstellung ist komplett anders ... “ (I27, männlich, *1991, seit 2005 in Ö.)

Indizien für einen Wertewandel lassen sich auch in Bezug auf die Einstellungen zu Gewalt nachweisen. Eine Frau berichtet, dass ihr im Herkunftsland zu Gewalttätigkeit neigender Ehemann sich nun zurückhält.

„,Und jetzt er macht nicht mit mir viel streiten oder Gewalt. Er wird ein bisschen netter ... Die Männer in Afghanistan ... alles machen jeden Tag. Gewalt mit seiner Frau, mit seiner Schwester, mit seiner Mutter, mit seinen Kindern. Immer jeden Tag Schlägerei und sowas. Aber hier man darf nicht Schlägerei machen und er kann nicht, Gott sei Dank, meine Kinder schlagen. " (I19, weiblich, *1973, seit 2011 in Ö.) 


\subsection{Religion und Glaube}

\subsubsection{Empirischer Forschungsstand}

Seit den Anschlägen vom 11. September 2001 zählt Afghanistan zu den Hauptzentren des internationalen islamistischen Terrors. Dementsprechend wird vielen Afghan/inn/en in Westen mit Misstrauen begegnet. Sie gelten als Vertreter/innen einer radikalen Religion, welche die Menschen- und Frauenrechte nicht achtet, säkulär-demokratischen gesellschaftspolitischen Strukturen skeptisch bis ablehnend gegenübersteht und eine stärkere Verankerung von Religion in Staat und Gesellschaft einfordern würde.

Afghan/inn/en wird, wie vielen anderen muslimischen Migrant/inn/en, unterstellt, dass ihre Wertvorstellungen primär durch den Islam und andere vormoderne Traditionen (z.B. hoher Stellenwert der Ehre, Segregation der Geschlechter, Verhüllung der Frauen) determiniert wären (vgl. El-Menouar 2017; SATTOR 2016). Dies wiederum stünde einer Integration in die österreichische Gesellschaft entgegen bzw. würde diese zumindest stark erschweren. Einzelne Boulevardmedien und rechte Politiker/innen betonen sogar die Gefahr, die von der Präsenz einer zu großen Zahl von muslimischen Flüchtlingen und Migrant/inn/en für die westliche Gesellschaft ausgehen würde. So behauptete beispielsweise die FPÖ Politikerin Nicole Hosp in einer Presseaussendung vom 7. Jänner 2016, „... dass die Immigration muslimischer Flüchtlinge durch die still und leise vor sich gehende Erodierung unserer Werte, die schwer erkämpften und geteilten Ansichten über die Frauen in unserer Gesellschaft gefährdet.“ (Hosp zitiert in SCHEIBELHOFER 2017, p. 102, eigene Übersetzung).

Als Musterbeispiele für die mangelnde Integrationsfähigkeit von Muslim/inn/ en wird in den öffentlichen Diskursen zudem immer wieder auf die Themenkomplexe, „Kopftuch/Burka“ sowie „Ehrenmord“ verwiesen. Insbesondere mit dem Zuzug zehntausender muslimischer Asylwerber/innen (v.a. aus Afghanistan, Syrien, Somalia und Irak) während der sogenannten Flüchtlingskrise im Jahr 2015 verstärkte sich die Debatte um die Integrationsproblematik von Muslim/inn/en und deren vermeintliche oder tatsächliche Unfähigkeit, sich auf Grund ihres Werte- und Normensystems friktionslos in die österreichische Gesellschaft zu integrieren.

Um ihre Werthaltungen und Einstellungen zu Religion und westlicher Gesellschaft sowie den hier geltenden Normen und Werten (z.B. Trennung von Religion und Staat, Gendergleichheit etc.) auszuloten, wurde seit 2017 eine Reihe von quantitativen Erhebungen unter muslimischen Flüchtlingen und Migrant/inn/en durchgeführt. Dabei wurden entweder Vergleiche zwischen einzelnen Flüchtlingsgruppen angestellt (vgl. KoHLbacher 2017; KoHLBACHER et al. 2017; BubER-EnNSER et al. 2018; Aslan 2018) oder rezente Flüchtlinge mit ehemaligen Flüchtlingen (z.B. aus Bosnien) und türkischen Migrant/inn/en der ersten und zweiten Generation verglichen (z.B. FILzMaIER und Perlot 2019; GÜNGÖR et al. 2020). 
Zentrale Themenbereiche, die in diesen Studien abgefragt wurden, bezogen sich auf den Stellenwert der Religion für die befragten Personen, die Rolle, die der Islam im öffentlichen Leben Österreichs spielen sollte, das Verhältnis von Religion und Politik, insbesondere in einer demokratischen Gesellschaftsordnung, sowie die Beziehungen $\mathrm{zu}$ Angehörigen anderen Religionsgemeinschaften und die Gleichberechtigung der Geschlechter.

Im Resümee zeichnen die bislang vorliegenden quantitativen Studien ein deutlich differenzierteres Bild als das von Teilen der Medien verbreitete und von rechtspopulistischen Politiker/inn/e/n postulierte Image des nichtintegrierbaren muslimischen Flüchtlings. Weder handelt es sich bei diesen um eine homogene Gruppe, die das westliche Gesellschafts- und Politikmodell sowie seine Normen und Werte ablehnt, noch besitzen der Islam oder andere Traditionen für alle eine gleich große Bedeutung.

Besonders gut kann diese Diversität in qualitativen Erhebungen wie der vorliegenden herausgearbeitet werden. In diesen geht es nicht um die Analyse von Zusammenhängen zwischen einzelnen Items (z.B. Selbsteinschätzung der eigenen Religiosität, Häufigkeit des täglichen Gebets, Tragen des Kopftuches etc.) und anderen Faktoren wie Alter, Geschlecht und Bildungsstand, sondern um ein umfassendes, kontextbezogenes Verständnis der zu untersuchenden Thematik (in diesem Fall des Stellenwerts von Religion).

Ein illustratives Beispiel ist der in zahlreichen quantitativen Studien abgefragte Indikatorenkatalog (z.B. Häufigkeit der täglichen Pflichtgebete, Besuch der Moschee, Tragen des Kopftuches etc.), der Aufschluss über den Stellenwert der Religion für die befragte Person und damit ihre Religiosität liefern soll. Warum jemand die täglichen Pflichtgebete oder den Moscheebesuch einhält oder verabsäumt, das Kopftuch trägt oder andere Bekleidungsvorschriften beachtet oder ignoriert, erschließt sich damit jedoch nicht.

Dafür ist es notwendig, auch das Umfeld, in welchem eine Person sozialisiert wurde, zu beleuchten bzw. die Erfahrungen, die Respondent/inn/en in einem spezifischen Kontext gemacht haben (z.B. Leben unter dem Taliban-Regime, Zugehörigkeit zu einer religiösen oder ethnischen Minderheit, z.B. schiitische Hazara, die im sunnitisch dominierten Afghanistan und Pakistan oft diskriminiert werden) in Betracht zu ziehen.

So lehnten beispielsweise einige unserer Respondent/inn/en, die während der Mujaheddin- oder Taliban-Herrschaft in Afghanistan sozialisiert oder im Iran aufgewachsen waren, ein enges Verhältnis zwischen Religion und Politik ab. Auch das Islamverständnis vieler Afghan/inn/en war und ist sehr stark durch das soziale Umfeld, in welchem sie lebten und leben, und die jeweiligen Rahmenbedingungen mitbestimmt. Gebildete Personen, die sich selbst als sehr religiös einschätzten und in einer Zeit aufgewachsen waren, in welcher der politische Islam in Afghanistan noch keine Rolle gespielt hatte, vertraten oft deutlich moderatere Ansichten in Bezug auf das Zusammenleben mit Angehörigen anderer Religionsgemeinschaften oder den Stellenwert der Frau in der Gesellschaft, als dies für Personen mit gleicher Schulbildung zutraf, die in 
einer Periode sozialisiert wurden, in welcher stärker islamistisch geprägte Diskurse an Einfluss gewannen, wie z.B. während der Taliban-Ära.

Derartige kontextbezogene Hintergrundinformationen können durch quantitative Erhebungen kaum generiert werden. Sie sind aber, wie die nachfolgenden Beispiele aus den Respondent/inn/en- und Expert/inn/eninterviews demonstrieren, essentiell für ein umfassendes Verständnis und eine aufschlussreichere Einordnung empirischer Befragungsergebnisse. Die Analyse der durchgeführten Interviews verdeutlicht darüber hinaus, dass die in Österreich lebenden Afghan/inn/en eine breite Palette unterschiedlicher Ansichten und Werte vertreten, die wenig mit dem in Medien und Politik gezeichneten Bild des/der ,,archaischen“ Afghan/e/i/n zu tun haben. Dies macht zudem deutlich, dass sich Werthaltungen und Einstellungen im Laufe der Zeit auch verändern können und dass das jeweilige Umfeld und die Lebenserfahrungen der Betroffenen eine wesentliche Rolle dabei spielen können.

\subsubsection{Analyse der Interviews}

\subsubsection{Wichtigkeit von Religion und religiöse Praxis}

\section{Pragmatischer und säkularisierter Umgang mit Religion}

Die Mehrheit der Respondent/inn/en gibt an, sich zum Islam zu bekennen, aber nicht strenggläubig zu sein. Die religiösen Praktiken, wie fünfmaliges tägliches Gebet, regelmäßiger Moscheebesuch oder das Fasten während des Ramadan werden oft nicht strikt eingehalten. Dafür werden verschiedene Gründe genannt. Ein wesentlicher ist, dass neben der Arbeit und sonstigen Pflichten oft die Zeit fehlt, häufiger zu beten oder die Moschee zu besuchen. Es scheint aber auch so zu sein, dass diese Praktiken in Österreich im Vergleich zum Herkunftsland generell an Relevanz und Bedeutung verlieren. Manchmal sehen die Respondent/inn/en einen Widerspruch zwischen den vorgeschriebenen religiösen Praktiken und den Anforderungen des Alltags in Österreich, z. B. ein/e gut/e Mitarbeiter/in zu sein, den Job nicht zu verlieren und die Familie zu ernähren.

„,Ich denke nicht so viel an Religion. Ich denke immer über Arbeit nach, über was machen ich für Zukunft und für meine Kinder. “ (I42, weiblich, *1992, seit 2014 in Ö.)

„, Ich gehe in die Moschee nicht immer, kann ich nicht, habe ich keine Zeit. Normalerweise muss man fünf Mal in die Moschee gehen und beten, aber das kann man zuhause auch. Einmal in der Woche, am Freitag, man muss gehen und wenn ich Zeit habe, gehe ich. Und das ist Freiheit, das ist für mich. " (I26, männlich, *1974, seit 2013 in Ö.)

„Ich glaube, als Moslem musst du das machen, was ein Moslem machen soll. Zum Beispiel fünf Mal beten. Was in unserem Buch steht: Fasten, alles - das muss man alles machen. Aber von einer anderen Seite, wenn ich denke, dass ich jetzt hier 
bin - ok, für unsere Religion ist es ganz, ganz wichtig, die Familie zu unterstützen. Ok, wenn ich hier fünf Mal bete, dann wird mich der Chef kündigen. Wenn er mich kündigt, wie kann ich dann meine Familie unterstützen? Das ist auch nicht gut. Deswegen mache ich es jetzt einfach so, ... Ich mache nur das, was ich gut finde. “ (I1, männlich, *1989, seit 2013 in Ö.)

Zusätzlich zum Beten und Fasten wurde bezüglich der Gebote des Islam besonders oft auch das Tragen eines Kopftuches angesprochen. Durch den Wegfall des gesellschaftlichen und oft auch familiären Drucks, ebenso wie durch eine gewisse Anpassung an die Gesellschaft in Österreich - teilweise bedingt durch die empfundene Diskriminierung von kopftuchtragenden Frauen - verliert das Kopftuch seine Bedeutung. Ob es getragen wird oder nicht wird zu einer persönlichen Entscheidung, die auch von anderen als solche anerkannt wird.

„,Wie ich nach Österreich gekommen bin, hat [mein Ehemann] gesagt, die Entscheidung liegt bei dir, ob du ein Kopftuch tragen willst, oder nicht, aber entscheide dich jetzt. Entweder du trägst es von Anfang an, oder du trägst es von Anfang nicht. Nicht dass du dann Jahre später dich umentscheidest und ich dann von meinen Freunden zur Rede gestellt werde, sodass sie sich lustig machen und sagen: ,Schaut euch die Frau von ihm an! "“ (I58, weiblich, *1986, seit 2016 in Ö.)

Ein weiterer Faktor, der zur Veränderung der Religiosität in Österreich beiträgt, ist der zusätzliche Informationshorizont, zu dem die Afghan/inn/en in Österreich Zugang haben. Dadurch werden sie dazu angeregt, religiöse Werte oder Praktiken nicht mehr „,blind“ zu akzeptieren, sondern eigenständig darüber nachzudenken, ob diese für sie Sinn machen und ob sie diese tatsächlich befolgen möchten.

„Jetzt [bin ich] weniger [religiös], weil früher hatte ich nicht so viele Informationen über die Religion, jetzt schon. Und ich habe neue und andere Erfahrungen gemacht und gesehen. " (I54, männlich, *1996, seit 2015 in Ö.)

„, Religion war sehr wichtig für mich. Bis 2010, 2011 oder so war ich sehr streng gläubig. Ich bin zu der Überzeugung gekommen, dass man auch selber nachdenken sollte und nicht immer alles sofort glauben sollte. " (I62, männlich, *1994, seit 2015 in Ö.)

Es wurde in den Interviews auch oft betont, dass die Religion zweitrangig ist und dass das Wichtigste in einer Art „Menschlichkeit“ besteht, d.h., dass man ein ,guter Mensch“ ist. Dazu gehören für viele Tugenden wie Ehrlichkeit, dass man Menschen nicht bestiehlt und dass man andere Personen gut behandelt. Höchste Priorität kommt aber der Friedfertigkeit zu, also, dass keine Gewalt ausgeübt wird und dass man anderen Menschen gegenüber mitfühlend ist.

„Bevor ich nach Österreich gekommen bin, hat sich in meinem Leben alles um die Religion gedreht. Ich musste alle Regeln befolgen, aber jetzt habe ich keine 
Religion. Ich bevorzuge Menschlichkeit anstatt Religion. “ (I55, männlich, *1998, seit 2014 in Ö.)

„, Religion ist für mich wie eine Leitung fürs Leben. Doch Religion kommt bei mir immer an zweiter Stelle. Am wichtigsten ist es für mich in erster Linie, dass wir alle Menschen sind. Wenn wir uns menschlich verhalten, verhilft uns das auch dazu, dass wir unsere Religion besser verstehen. " (I65, männlich, seit 2015 in Ö.)

„,Meiner Meinung nach, ob ein Mensch gut oder böse ist, hängt nicht von der Religion ab. Man muss es in der Tat beweisen. Zum Beispiel man soll nicht stehlen oder nicht lügen. Ob man betet oder fastet, sagt lange nicht über die Persönlichkeit des Menschen. Es gibt Menschen, die beten und fasten und dann lügen und stehlen oder sowas. “ (I47, männlich, *1996, seit 2015 in Ö.)

\section{Distanzierung und Abwendung von Religion}

Die Interviews weisen häufig auch auf eine Relativierung bis hin zu einer Distanzierung und Abwendung von Religion hin.

„Natürlich bin ich jetzt weniger religiös. Ich habe mich damals nicht immer über eine Sache geäußert. Jetzt traue ich mich, meine Meinung zu sagen. “ (I50, männlich, *1991, seit 2015 in Ö.)

„In Afghanistan waren wir gezwungen zu beten. Hier weder bete ich noch andere Dinge, aber ich glaube an die Religion. “(I28, weiblich, *1977, seit 2013 in Ö.)

Manche haben sich aber auch komplett von der Religion abgewandt, unter anderem deswegen weil sie diese als zu eng mit der Politik verbunden sehen.

„Das muss man ehrlich sagen, also das ganze, die ganze Religion, ist ja nur Politik. “ (I22, männlich, *1969, seit 1986 in Ö.)

Die Expert/inn/en bestätigen, dass sich viele aufgrund der schlechten Erfahrungen in der Vergangenheit von der Religion abwenden.

„, Viele haben da eine kritischere Einstellung. Da sagen: ,Zu viel Religion ist nicht gut'. "(E2)

„Sie sind junge Männer, die einfach genug haben vom Islam, weil die sagen: ,Ok, unser Regime, ob es halt in Afghanistan oder im Iran ist, ist vollkommen egal, nutzt das, um uns zu unterdrücken ... "“ (E4)

Festhalten an der Religion und gesteigertes Interesse

Die Einschätzung eines Experten aus dem religiösen Bereich ist, dass es „viele“ religiöse Flüchtlinge und Afghan/inn/en in Österreich gibt, die auch die Moschee besuchen.

„Am Freitag zu uns kommen sehr viele Leute, also das sind zirka 400 Leute, was dann kommen zum Gebet und das sind fast 90\% junge Leute. Die sind ja auch großteils Flüchtlinge. “(E7) 
Zumindest in einigen Interviews werden die Wichtigkeit des Glaubens und die ausgeprägte Religiosität bekräftigt.

„Ich halte dies [religiösen Praktiken und Gebote] für sehr wichtig und ich glaube so ergeht es vielen Menschen, egal welcher religiösen Zugehörigkeit. Man wird ja nicht dazu gezwungen, die meisten machen es freiwillig und aus tiefster Überzeugung. Man kann niemanden zu so was zwingen. Ich persönlich bin sehr glücklich damit, dass ich eine Muslimin bin und fühle mich sehr wohl dabei. Und auch die religiösen Feierlichkeiten genieße ich. “ (I57, weiblich, *1966, seit 2015 in Ö.)

Bei einigen hat sich das Interesse für Religion und ihre Religiosität seit ihrem Aufenthalt in Österreich gesteigert. Die Vermutung liegt nahe, dass die Respondent/inn/ en auf diese Weise einen Weg suchen, um mit ihrer Herkunft verbunden zu bleiben. Die Religion dürfte also bei diesen Personen ein wichtiger Teil ihrer Identität sein.

„Ich glaube, in Afghanistan war ich weniger religiös. “ (I52, männlich, *1993, seit 2009 in Ö.)

„Ja, fasten und beten und alles sind wichtig für mich, weil ich Muslima bin und darf nicht meine Kultur verlernen. In meiner Familie, meine Mutter ist religiöser als ich und sie hätte es gerne, dass wir so wie sie sind. " (I59, weiblich, *1999, seit 2016 in Ö.)

Für manche stellen die Ausübung der Religion und das Beten eine seelische Stütze oder Strategie dar, um mit Enttäuschungen und schwierigen Situationen klarzukommen (Coping Strategy). In einigen Interviews wurde die Religion mit Psychotherapie verglichen, speziell in schwierigen Situationen, wie etwa beim Verlust eines geliebten Menschen.

„Für mich ist wichtig, weil es hat mir so viel geholfen. ... Es hat für mich viel verändert. Ich fühle mich, Beispiel, in Ruhe, wenn ich bete." (I24, weiblich, *1996, seit 2015 in Ö.)

„Und da finde ich dann wieder Religion gut. Weil zum Beispiel als meine Mutter verstorben ist, da hätte, glaube ich, die teuerste oder die beste Psychotherapeutin nicht helfen können, und ich war mir 100\% sicher, dass sie mir nicht helfen konnte. ... Weil eigentlich Religion ist gekommen in dem Sinne, wenn die Religion nimmt, um das Leben von Menschen zu erleichtern, um gewisse Wege zu zeigen." (I4, männlich, *1985, seit 2001 in Ö.)

Laut Expert/inn/en kann diese Religiosität die Integration sowohl unterstützen als ihr auch im Wege stehen, etwa wenn sie mit Abschottungstendenzen verknüpft wird. Für manche spielt die eigene Religion insbesondere zu Beginn ihrer Zeit in Österreich eine wesentliche Rolle. Expert/inn/en haben jedoch auch beobachtet, dass sich junge Geflüchtete in Österreich zuerst von ihrer Religion abwenden, später jedoch wieder zur Religion finden, weil sie Halt und Orientierung suchen. 
„Religion kann gerade am Anfang etwas sein, das Halt gibt und somit Teil der Lösung sein kann, man dadurch auch Leute kennenlernt und so weiter. Problematisch wird es, wenn die Religion den Integrationskontakt zu behindern beginnt, dass dann der Kontakt nur in der eigenen Community/Glaubensgemeinschaft bleibt. Wenn es Abschottungstendenzen gibt, also ein ,Wir gegen sie'-Narrativ, dann wird es eher zum Problem. "(E13)

„Die Jugendlichen sind meistens nicht besonders religiös bis ... Manche, die dann wieder zu einer Religiosität zurückkommen nach Phasen des Schweinefleisch-und Alkoholkonsums oder so. Die merken, das tut ihnen vielleicht nicht gut. Die dann so einen Halt suchen in der Religion, was auch so ein typisches Exilphänomen ist ein bisschen. " (E2)

\subsubsection{Mangel an religiöser Bildung}

Ein zentrales Problem scheint ein Mangel an religiöser Bildung und Zugang zu Informationen zu sein. Darauf weisen sowohl Respondent/inn/en als auch Expert/inn/ en hin und sehen darin eine Quelle von Integrationsproblemen und Radikalisierungstendenzen.

„,Viele Afghanen wissen nicht viel von Religion und wenn sie was wissen, dann nur von Erzählungen und von den Medien. “ (I34, männlich, *1978, seit 2012 in Ö.)

„Das heißt man ist angeboren als Muslim und man hat so viel Ahnung vom Islam, wie man in der Familie mitbekommen hat. Papa betet, bete ich auch. Mutter sagt das, dann passt das so. "(E6)

„Das ist eigentlich auch so ein Spiel der Religion und diese Imame, meiner Meinung nach, in Afghanistan und viele sind nicht richtig gebildet und deswegen gibt es auch diese falschen Interpretationen für die Menschen, weil da hört man, was der Imam sagt, passt schon. Aber was der Islam richtig für mich sagt, das wissen sie nicht oft. Auch bei diesen ganzen Radikalen, die alles Mögliche ausüben in Europa, die wissen nicht, was sie alles eigentlich tun. Die wissen nicht, dass sie eigentlich die größte Sünde begehen, indem sie einfach unschuldige Menschen umbringen. "(E6)

Auch auf die religiöse Bildung verweisend, unterstreicht eine Expertin die Wichtigkeit der Ausbildung der Imame in Österreich, insbesondere im Hinblick auf einen Islam, der mit europäischen Grundwerten vereinbar ist:

„Ein wichtiger Punkt ist die Ausbildung von Imamen im Inland. Die Uni Wien hat mittlerweile ein Institut für Islamisch-Theologische Studien, das im Inland Imame ausbildet. Das ist ein wichtiger Schritt, dass im Inland die Ressourcen bereitgestellt werden und man sich nicht darauf verlassen muss, was aus dem Ausland kommt. Somit wird der Einfluss aus dem Ausland eingedämmt. Jemand der hier aufgewachsen ist und seine Ausbildung hier genossen hat, hat mehr Bezug zum Leben in diesem Land als jemand, der für zwei Jahre zu Besuch kommt. Das 
sind nicht Themen, die primär für die afghanische Community wichtig sind, aber generell auch bei Gruppen, die schon länger hier sind, ist das ein großes Thema, vor allem dann, wenn Religion staatlich organisiert ist. Dadurch wird auch dem Glauben die Möglichkeit geboten, sich im Inland zu entwickeln, aus integrationspolitischer Sicht auch im Sinne eines Islams europäischer Prägung. “ (E13)

\subsubsection{Moscheevereine}

In den Interviews wurde des Weiteren nach der Rolle und Bedeutung von Moscheevereinen in Österreich gefragt. Einige stehen den Moscheevereinen positiv gegenüber. Die Mehrheit allerdings interessiert sich kaum für Moscheen und besucht diese auch nicht regelmäßig. Dennoch wird die Existenz von Moscheen sowie das Recht, in Österreich Moscheen zu bauen, als Zeichen einer offenen, toleranten und vor allem freien Gesellschaft gesehen. Für viele religiöse Afghan/inn/en sind Moscheevereine nicht von besonderer Bedeutung, weil sie ihre Religiosität mehr in ihrer persönlichen Beziehung zu Gott sehen und somit nicht auf Moscheen als „Vermittler“ angewiesen sind. Insbesondere anlässlich von Festen und besonderen Anlässen wie Trauerfällen, spielen Moscheen eine sozial und kulturell bedeutsame Rolle.

„Zum Beten nein, aber falls ich zu einer Trauerfeier eingeladen sein sollte, dann schon. Denn das Leid meiner Landesmänner ist auch mein Leid. " (I64, männlich, *1950, seit 1983 in Ö.)

„Also ich gehe nicht wirklich oft in die Moschee. Nur selten, wenn irgendetwas wichtiges ist. “ (I65, männlich, seit 2015 in Ö.)

\section{Probleme im Zusammenhang mit Moscheevereinen}

Einige Respondent/inn/en distanzieren sich sehr explizit von Moscheen und meinen unter anderem, sich dort aufgrund der strikten Regeln nicht wohlzufühlen. Es wurde auch angemerkt, dass man aufpassen müsse, mit wem man dort redet, weil erstens hinter dem Rücken über einen gelästert wird und auch weil sich Personen mit einer „strengeren“ religiösen Sichtweise dort aufhalten.

„Ich möchte, dass die Anzahl der Kulturvereine steigt und bin dagegen, dass die Anzahl der Moscheen und islamischen Zentren steigt. Wenn jemand religiös sein möchte, dass sieler es gerne zuhause machen und braucht nicht unbedingt eine Moschee dafür, damit sich 40 bis 50 Menschen sammeln und dann auch beim Verlassen des Orts die Bewohner nerven und Unruhe stiften. " (I48, männlich, *1991, seit 2014 in Ö.)

„, Weil ich gehe nicht, weil das ist gefährlich für unsere Kinder. Wenn sie einmal zur Religion gehen, das macht gefährlich. Wenn sie zu Moschee gehen, dann Mullah sagt: ,Machst du so, machst du so!', dann ausnützen unsere Kinder. Wir lassen nicht unsere Kinder in Moschee, nie. " (I19, weiblich, *1973, seit 2011 in Ö.)

Die Gefährdung der Integration, die von Moscheevereinen ausgehen kann, wird auch von einer Expertin beschrieben: 
„Eine Studie zur Rolle der Moschee im Integrationsprozess hat durchaus besorgniserregende Ergebnisse zutage gebracht hat, nämlich, dass einige Moscheen der Integration bestenfalls nicht im Wege stehen und dass einige doch die Integration behindern - aufgrund eines Nationalismus, der vermischt wird mit der Religion. Das kann auch auf die zweite Generation übergehen, dass die Verbundenheit stärker zum Herkunftsland unterstrichen wird, wo das stark mit einer Religiosität vermischt wird, zum Beispiel: ,Du kannst nur gläubiger Moslem sein und kein Österreicher, ' aber das betrifft nicht nur die afghanische Community. “ (E13)

\section{Positive Rolle in der Integration}

Moschee- und in einem höheren Ausmaß Kulturvereine können den Afghan/inn/ en auch einen sozialen Rückhalt bieten sowie bei praktischen Anliegen helfen und auch als Vernetzungsort für die Community wirken. Durch die soziale und praktische Unterstützung erhalten relativ rezent angekommene Afghan/inn/en wichtige Informationen und es kann ihnen auch bei der strukturellen und sozialen Integration geholfen werden, zum Beispiel durch das Organisieren einer Wohnung oder Arbeit.

„,... diese Vereine ermöglichen diese Community zusammenzuhalten. ... Oder als ich zum Beispiel geheiratet habe, auch war die Community da. Die haben mich finanziell unterstützt, auch bei der Feier unterstützt, alles zu organisieren, alles reibungslos zu laufen. Und das hat alles funktioniert. “ (I4, männlich, *1985, seit 2001 in Ö.)

„Also Bildung, da können die Vereine schon was helfen. Das ist auch eine der Dinge, die diese Interessengemeinschaft der afghanischen Schüler/innen und Studierenden massiv macht, Vorträge zum österreichischen Bildungssystem ... “ (E2)

„Also ich glaub es gibt ein paar Kulturvereine, afghanische Kulturvereine, die halt auch versuchen, die Leute irgendwie zu orientieren und in Kontakt zu bringen mit Österreichern ... “ (E5)

\subsubsection{Haltung gegenüber anderen Religionen und multireligiöses Zusammen- leben}

Unabhängig von der eigenen Einstellung zu Religion und Glauben sprechen sich alle Respondent/inn/en explizit für die Religionsfreiheit aus, egal ob sie religiös sind oder nicht. Sie sind glücklich darüber, dass die, die das wollen, auch hier ihre Religion - ganz gleich welche - ausüben können.

„Das ist sehr gut, das ist erlaubt ..., da kann man ein Fest feiern oder in die Moschee gehen, das ist Freiheit. Das ist sehr wichtig. “ (I26, männlich, *1974, seit 2013 in Ö.)

Diese hohe Wertschätzung der Religionsfreiheit wurde auch in den Expert/inn/ eninterviews bestätigt: 
„,Und da zeigt sich, dass einerseits zum Beispiel Afghanen, Muslime generell, die befragt worden sind, die Religionsfreiheit in Österreich sehr schätzen. “ (E6)

Es wurde auch dahingehend für die Religionsfreiheit argumentiert, dass Religion und Glaube Privatsache sind und dem Individuum keine Regeln von außen aufgezwungen werden sollten.

„, Religion sollte sich nicht in Gesetze einmischen. Weil es gibt immer die Leute, die Religion benützen. Eigentlich finde ich, Religion ist eine Privatsache. " (I17, männlich, *1997, seit 2013 in Ö.)

Generell sprechen sich die Respondent/inn/en für die Toleranz gegenüber anderen Religionen und ein friedliches Zusammenleben aus. Dies passt auch zur positiven Einstellung gegenüber der Religionsfreiheit in Österreich, wie bereits oben beschrieben.

„Also, ich persönlich, also ich bin vom Islam überzeugt, dass das die Wahrheit ist. Aber genauso ich toleriere alle Religionen. “ (I45, weiblich, *1987, seit 1992 in Ö.)

„,Wenn ich jemanden kennlerne, will ich ihm wie ein Freund begegnen und auch Respekt für seinen Glauben haben. “ (I65, männlich, seit 2015 in Ö.)

Ein weiterer wichtiger Punkt ist der Vergleich des Islam mit anderen Religionen. So wurde in den Interviews öfter argumentiert, dass es egal ist, welche Religion jemand hat, weil sich im Grunde genommen alle um den gleichen Gott drehen und es auch viele Gemeinsamkeiten in den Wertehaltungen gibt.

„Ein Herzenszustand, d.h. Islam ist Zustand, ein geistiger, ein spiritueller Zustand. Und wenn man das so interpretiert und merkt, dass Spiritualität es auch im Buddhismus und Christentum und Hinduismus gibt und dass die Philosophie eh ungefähr gleich ist, dann merkt man, da kommt man weg von dem Extremismus. “ (I5, männlich, *1996, seit 2006 in Ö.)

\subsubsection{Reaktionen auf Kritik an der Religion}

Wenn es um die Kritik an der eigenen Religion geht, hängen die Reaktionen stark davon ab, was kritisiert wird und wie diese Kritik geäußert wird. Generell haben die meisten gegen eine fundierte Kritik nichts einzuwenden.

„,Auch Religion ist ein Teil der Gesellschaft, und man darf auch die Religion kritisieren. Und es ist genauso, wie wenn auch Politiker kritisieren oder Sozialwissenschaftler. Religionen darf man auch kritisieren. “(I30, männlich, *1953, seit 1984 in $\ddot{O}$.)

„Ich finde Kritik grundsätzlich OK. Ich finde, es ist das Recht eines jeden Menschen immer den anderen zu kritisieren. Darin sehe ich überhaupt kein Problem. Nur halt, ich finde, man soll aber immer versuchen, höflich und respektvoll zu sein. “ (I5, männlich, *1996, seit 2006 in Ö.) 
Die mediale Berichterstattung in Österreich, die als generalisierend und voreingenommen empfunden wird, wird allerdings als Problem betrachtet, da sie das Image der Afghan/inn/en negativ beeinflusst und sich somit auf viele Lebensbereiche nachteilig auswirkt. Es wird auch unterstrichen, dass es sich bei den Afghan/inn/en, die negativ auffallen, um Einzelfälle handelt, die nicht gemäß den Prinzipien des Islam handeln, sondern entsprechend einer fehlerhaften Interpretation.

„Aber manchmal stört mich, dass die anderen Leute vielleicht glauben, wenn muslimische Leute negative Sachen oder schlechte machen, dann bin ich traurig, dass alle Leute denken, vielleicht sind alle muslimischen Leute so. Aber das ist nicht so. “ (I35, weiblich, *1974, seit 2004 in Ö.)

„,... wenn jetzt im Fernsehen der Islam kritisiert wird, weil er meint, es ist nicht der Islam, der mangelhaft ist, sondern es sind die Muslime, die die Fehler machen. Also wenn man sie kritisiert, dann wirklich die Taten der Muslime. " (I43, männlich, *1991, seit 2012 in Ö.)

\subsubsection{Religiöse Feste als kulturelle und soziale Anlässe}

Die religiösen Feste sind für viele Afghan/inn/en in Österreich ein Anlass um zu feiern, sowie Freunde und Familienangehörige zu treffen. Der soziale Aspekt steht im Vordergrund, während die religiöse Bedeutung eher nebensächlich zu sein scheint.

„, In erster Linie aus dem Grund, weil das ein soziales Fest ist. Allein die Menschen zu sehen und mit den Menschen zu sprechen, kommunizieren, das ist etwas, was für mich viel wichtiger ist als der Inhalt dieser Feste selbst. Das ist eine Art Vorwand, das nehme ich zum Anlass, um wirklich die Menschen zu sehen und mit den Menschen gewisse soziale Kontakte zu pflegen. " (I37, männlich, *1952, seit 1988 in Ö.)

„Ja, schon, zu Festen und so. Ich denke mir auch, damit die Kinder ein bisschen was von unserer Kultur lernen ... Aber sie feiern Weihnachten, sie feiern auch unsere Feste. Ich denke, sie können beides, weil sonst vergessen sie das. Und wenn wir halt zuhause sind und die Oma was erzählt, dann wissen sie: ,Ok, die haben diese Tage dort auch gefeiert. "“ (I32, weiblich, *1975, seit 1995 in Ö.)

\subsection{Demokratie und Rechtsstaat}

\subsubsection{Empirischer Forschungsstand}

Vielfach wird Muslim/inn/en in öffentlichen Debatten insbesondere von Seiten rechtspopulistischer Politiker/innen unterstellt, dass sie die grundlegenden Werte und Prinzipien der österreichischen Rechts- und Gesellschaftsordnung ablehnen würden und stattdessen für die Einführung des islamischen Rechts und/oder die Etablierung eines islamischen Staatswesens plädieren würden. 
Die wenigen seit 2015 in Österreich durchgeführten quantitativen Studien belegen jedoch, dass die überwiegende Mehrheit der hier lebenden Afghan/inn/en, wie auch andere muslimische Flüchtlinge und Migrant/inn/en, eine Trennung von Staat und Religion befürwortet (vgl. Kohlbacher et al. 2017; Filzmaier und Perlot 2017). Für sie stellt die Demokratie die ideale Staatsform dar. Begründet wird dies u.a. damit, dass der demokratische Rechtsstaat die freie Religionsausübung garantiert, die Menschenrechte geachtet werden und alle vor dem Gesetz gleich sind. Insbesondere die Rechtsstaatlichkeit westlicher Demokratien und die gewaltfreie Austragung politischer Meinungsverschiedenheit wird hochgeschätzt, zumal diese für viele im Herkunftsland nicht gegeben war.

Obwohl also das demokratische System und der österreichische Rechtsstaat positiv bewertet werden und die überwiegende Mehrheit der Afghan/inn/en für eine Trennung von Religion und Politik eintritt und auch kein stärkeres Mitspracherecht der Religion innerhalb des österreichischen Staates einfordert, haben einzelne Afghan/inn/ en (vor allem jenen mit nur rudimentärer Schulbildung), wie die nachfolgende Analyse der Respondent/inn/en und Expert/inn/en-Interviews illustriert, bisweilen Schwierigkeit genau zu verstehen, wie eine westliche Demokratie funktioniert bzw. was genau unter dem Begriff Säkularität zu verstehen ist. Beides dürfte eng mit dem Kontext, in welchem afghanische Flüchtlinge sozialisiert wurden bzw. den Erfahrungen, die sie im Herkunftsland gemacht hatten, zusammenhängen.

In Afghanistan gab es selbst in Zeiten, in denen der politische Islam keine Rolle spielte und sich die Staatsführung an westlichen Idealen orientierte (z.B. während der 1960er und 1970er Jahre), keine strikte Trennung zwischen Staat und Religion. Bis heute darf die staatliche Gesetzgebung den Grundprinzipien des Islams nicht zuwiderlaufen (vgl. TARZI 2012; RASULY 1993, 1997). Zudem ist die afghanische Gesellschaft, wie viele andere vormoderne Sozietäten, sehr stark durch Normen und Werte geprägt, die durch die Religion und andere lokale Traditionen beeinflusst sind. Diese stellen nicht nur eine Richtschnur für das Verhalten des/der Einzelnen sowie der Gesamtgesellschaft dar, sondern gelten auch als essentielle Bestandteile der afghanischen Identität. Daraus resultiert das spezifische Verständnis der Beziehung zwischen Religion, Gesellschaft und Politik, das für einen Teil der nun in Österreich lebenden Afghan/inn/en relevant ist.

Was die Einstellung zur Demokratie und zum eigenen politischen Engagement angeht, so ist diese sehr stark durch die im Herkunftsland gemachten Erfahrungen geprägt. Trotz der Verabschiedung einer neuen Verfassung (2004), die den Bürger/inn/ en des Landes gleiche Rechte gewährt, und der mehrfachen Abhaltung von Parlamentsund Präsidentenwahlen, basiert das politische System Afghanistans im Wesentlichen auf politischen Klientelnetzwerken, die einander gegenseitig bekämpfen und denen es primär um ihren eigenen Machterhalt geht. Der Etablierung demokratischer und rechtsstaatlicher Strukturen wird kaum Bedeutung beigemessen. Dies ist mit ein Grund dafür, dass viele nun in Österreich lebende Afghan/inn/en eine kritische Haltung gegenüber 
Politiker/inne/n einnehmen und selbst wenig Interesse an einem direkten Engagement in der Politik oder an politischen Debatten äußern.

\subsubsection{Analyse der Interviews}

In der vorliegenden Studie wurde untersucht, was die afghanischen Asylberechtigten in Österreich unter Demokratie und Rechtsstaatlichkeit verstehen und wie ihre Einstellungen dazu sind.

\subsubsection{Bedeutung von Demokratie}

Demokratie wird von den Befragten oft mit Freiheit assoziiert, solange diese die Rechte und Freiheiten anderer nicht verletzt. Diese Freiheit beinhaltet unter anderem die freie Religionsausübung und die freie Kleidungswahl, sofern man damit keine Grenzen überschreitet. Des Weiteren bedeutet Demokratie für viele, dass alle die gleichen Rechte haben.

„,Also Demokratie ist ... wie soll ich es ausdrücken ... das ist der Grundstein von einer Gesellschaft würde ich sagen. Und gerade in solchen Zeiten ist es umso wichtiger, dass Demokratie auch wirklich gegeben ist und dadurch die Meinungsfreiheit und Religionsfreiheit, Medienfreiheit, all das gehört für mich dazu. Und zu einer Demokratie gehört für mich auch dazu, dass man nicht nur davon spricht, dass Männer und Frauen gleichgestellt sind, sondern dass es auch gelebt wird in der Gesellschaft. Und für mich gehört zu einer Demokratie auch dazu, dass man, wenn man jetzt die Menschenrechte weiterbringen möchte, dass man bei den Frauenrechten ansetzt und die Frauenrechte voranbringt. Und dadurch kann man die Menschenrechte vorantreiben. " (I9, männlich, *1993, seit 2007 in Ö.)

Bei der Demokratie geht es den befragten Afghan/inn/en aber auch sehr um die „Menschlichkeit“ im Sinne vom Respekt vor der Menschenwürde und den Menschenrechten, um eine gerechte Gleichbehandlung aller und um die Rücksichtnahme auf die Mitmenschen statt einer grenzenlosen, absoluten Freiheit. Dies sehen viele Respondent/ inn/en als den besten Weg an, um eine sichere Gesellschaft und ein friedvolles, harmonisches Miteinander zu garantieren.

„, [An der Demokratie gefällt mir] dass hier jede/r als Mensch betrachtet wird und wir alle gleich sind und uns respektieren. Es ist nicht so wie im Iran. " (I29, weiblich, *1990, seit 2015 in Ö.)

„,Demokratie ist nicht, dass du alles machen darfst, was du willst. Eine demokratische Gesellschaft bedeutet, dass wenn jemand deine Rechte verletzt, du aufstehst und deine Rechte dir holst und kannst frei leben. Du darfst nicht das friedliche Zusammenleben in der Gesellschaft beschädigen ... " (I48, männlich, *1991, seit 2014 in Ö.)

Laut Expert/inn/en fällt es vielen Afghan/inn/en zu Beginn schwer, die Demokratie in Österreich oder Europa zu begreifen, da sie in Afghanistan, Iran oder Pakistan weder 
eine Demokratie erlebt haben, noch Zugang zu Informationen darüber hatten. Es ist teilweise schwierig, die Bedeutung von „Demokratie“ sowie die alltägliche Praxis zu vermitteln. Dies lässt sich aus der politischen Situation der Herkunftsländer erklären, wo es strikte Regeln für alle Lebensbereiche gibt. In einer Demokratie zu leben ist eine ganz neue Lebenserfahrung und deshalb besteht hierzu ein beträchtlicher Informationsbedarf.

„Eine Schwierigkeit ist, dass viele Afghanen - glaube ich - nicht ganz verstehen, wie unsere Demokratie funktioniert oder wie die Trennung zwischen Religion und Staat funktioniert. Also Gebildete sicher, aber Analphabeten nicht, weil in Afghanistan auch in der Zeit vor dem Talibanregime - also 1964 war ja die liberalste Verfassung mit den ersten umfassenden Parlamentswahlen - es war immer so, dass weder die Verfassung noch die staatlichen Gesetze irgendwie der Scharia zuwiderlaufen. "(E1)

„, Und dann natürlich sind Menschen gekommen und haben gesagt: , Wissen Sie, es ist so schwer, in einer Demokratie zu leben, weil ich muss immer alles selber ... ‘ Das heißt, sie müssen selber für sich entscheiden, [lacht] selber-sagen wir so-, selber schuld, wenn etwas nicht passt. [lacht] Sie können nicht jemand anderen dafür ... beschuldigen und sagen: ,Wegen dem das hat nicht geklappt. 'Dann kommen sie irgendwohin und dann dürfen sie sich alles selber machen. Wenn es nicht klappt, dann sind sie selber schuld. “(E8)

„, Und wenn sie hören, dass es in Österreich Religionsfreiheit gibt und in Europa generell und überhaupt auf der Welt gibt's Länder, wo man selber wählen kann, welche Religion man habe möchte und Religion mit Demokratie, mit den Gesetzen überhaupt nichts zu tun hat, das ist fremd. Das ist fremd für diese Menschen. Da müssen sie erst: ,Wirklich?! Ist das wirklich so?'Viele freuen sich. “(E4)

Hier schlagen die Expert/inn/en vor, die Wertekurse entsprechend zu erweitern bzw. mehr Zeit dafür vorzusehen und eine kontinuierliche Begleitung und Unterstützung in den ersten Jahren nach der Ankunft anzubieten.

\subsubsection{Einstellungen zur Demokratie}

Die Demokratie in Österreich wird von allen Respondent/inn/en als überaus positiv betrachtet. Trotz der Mängel und Verbesserungspotentiale im System, ist es für viele die beste Regierungsform, die sie kennen. Die mit der Demokratie einhergehende Entscheidungs- sowie Religions- und Meinungsfreiheit wird ebenfalls sehr geschätzt. Alle Interviewten sind sehr glücklich darüber, dass sie ihre Religion frei ausüben dürfen, falls sie das möchten und dass sie zu keinen religiösen Praktiken, egal welcher Religion, gezwungen werden.

„Und es ist auch der Demokratie zu verdanken, dass muslimische Frauen hier Kopftücher tragen können. Es ist Demokratie, dass die Muslime hier beten und fasten können und einfach frei ihre Religion ausüben können. " (I63, männlich, *1965, seit 2001 in Ö.) 
„Also ich bin ein ausgesprochener Demokrat. Ich finde das System fantastisch. Natürlich hat dieses System viele Fehler, aber eine Alternative kenne ich nicht. Es ist eigentlich die beste Art das Zusammenleben in einer Gesellschaft, eine Demokratie. “ (I30, männlich, *1953, seit 1984 in Ö.)

„Ich bin sehr positiv von der freiheitlichen Demokratie in Österreich beeindruckt, denn seit ich hier gekommen bin, merke ich, dass auch meine Kinder sich hier sehr wohl fühlen und in Freiheit leben. Unter Freiheit verstehe ich, dass man nicht belästigt wird. Ich kann ein Kopftuch tragen, meine Religion frei ausüben und werde darin von niemandem behindert. “ (I57, weiblich, *1966, seit 2015 in Ö.)

Es gab aber auch einige wenige Respondent/inn/en, die andere Erwartungen bezüglich der Freiheit in Österreich hatten.

„Im Iran dachte ich, dass man hier viel freier wäre. Seitdem ich hier bin, sehe ich, dass es nicht so ist, wie ich vermutet hatte. " (I50, männlich, *1991, seit 2015 in Ö.)

„, Religion unterrichten in der Schule - das ist Gewalt. ... Religion unterrichten in der Schule bis 18 muss streng verboten sein. “ (I11, männlich, *1988, seit 2011 in Ö.)

Zusätzlich zur positiven Einstellung gegenüber der Demokratie weisen Expert/ inn/en auch darauf hin, dass die Demokratie für manche nebensächlich ist, solange sie ein gutes Leben in Sicherheit führen können.

„,... wenn tatsächlich so ein Fluchthintergrund gegeben war, dass die natürlich auf keinen Fall in so einer Anarchie leben wollten. ... Also ich hab' eher das Gefühl gehabt, es ist ihnen darum gegangen, in geordneten Verhältnissen zu leben und dass das die Qualität ist, die sie haben wollen. Das ist es eher, sie wollten geordnete Verhältnisse und Sicherheit. " (E9)

„Also ich glaube schon, dass die Leute die Bildungschancen und auch die Demokratie ein bisschen überschätzen und die Möglichkeiten, die man da hat. Wobei, für manche ist das auch, genauso wie es für den Großteil der Österreicher, ist das auch wurscht einfach, weil die wollen leben und die wollen arbeiten und die wollen eine Wohnung. " (E2)

Darüber hinaus wird auch die Infrastruktur geschätzt, die mit einem funktionierenden Staat einhergeht, und die friedlichen Konfliktlösungen seitens der Politik.

„In Österreich gibt es wirklich Demokratie. ... alle da haben die Gleichberechtigung. .. ich denke, hier ist kein Rassismus. Alle Leute können ihr Leben normal haben und Religion haben oder so. Und bei Regierung ist auch eine gute Politik, weil wir bekommen jeden Monat Geld von Regierung, wir haben... für unsere Kinder Schule und Kindergarten und viele Verkehrsmittel haben wir und wenn wir Probleme bekommen, wir können Jugendamt gehen und fragen oder Sozialamt oder AMS oder so. Wir haben viele Möglichkeiten hier. Das ist wirklich demokratisches Land. " (I19, weiblich, *1973, seit 2011 in Ö.) 
„Ja, das Positive ist, dass sie im Parlament streiten, und dann aber im Gasthaus tun sie: ,Prost!' Das ist das Positive, dass die Argumente kommen. Muss man halt sehen, wer bessere Argumente, wer gut darstellen kann, wer positiv darlegen kann, natürlich, ist OK. “ (I38, männlich, *1951, seit 1974 in Ö.)

\subsubsection{Probleme bei der Umsetzung von Demokratie: Parteipolitik, Wahlver- sprechen, Populismus}

Infolge der Integrationshürden, seien es administrative oder soziale, sowie durch die erlebte Diskriminierung von Muslim/inn/en in der Politik und im Alltag wird die Ernsthaftigkeit und Aufrichtigkeit der Demokratie angezweifelt und von vielen Respondent/inn/en als unvollständig oder nicht zur Gänze implementiert angesehen. Dies führt zu Problemen bei traumatisierten Personen, die zunächst medizinisch-psychologische Hilfe benötigen, bevor sie mental und intellektuell überhaupt in der Lage sind, demokratische Werte zu rezipieren.

„, Auf der anderen Seite gibt es eine Gruppe, für die ist alles kaputt, alles tot, alles nicht einfach, da kann auch keine Veränderung kommen, weil die sind sehr stark psychisch belastet, die brauchen ärztliche Unterstützung, die sind psychisch nicht bereit die Gerechtigkeit, Demokratie, das alles zu hören, zu lesen, mitzumachen und auch anzunehmen. " (E12)

„Ich verstehe unter Demokratie, dass man sich um die Bedürfnisse des Volkes kümmert. Doch ich bezweifle, dass die Regierungen, nur darauf ausgerichtet sind die Interessen des Volkes zu vertreten. ... Nicht einmal indirekt helfen sie. " (I64, männlich, *1950, seit 1983 in Ö.)

„Es ist nicht eine komplette Religionsfreiheit. ... Es ist offensichtlich, dass viele kein gutes Bild haben von den Muslimen. Soweit ich weiß und gehört habe, ist es auch nicht leicht, ein Kopftuch zu tragen. " (I49, männlich, *1997, seit 2015 in Ö.)

Als ein Nachteil der Demokratie wird die Parteipolitik genannt, dass nämlich Politiker/innen ihre Wahlversprechen nicht einhalten und dass sie sich nicht um die wesentlichen gesellschaftlichen Probleme kümmern. Außerdem wird bemängelt, dass Geflüchtete und insbesondere Muslim/inn/e/n generalisierend negativ dargestellt und dazu benutzt werden, um mehr Wählerstimmen anzuwerben. Ein weiterer Kritikpunkt manifestiert sich in der Asylpolitik und in den Abschiebungen von Afghan/inn/en, da Afghanistan keinesfalls als sicheres Land betrachtet wird.

„Aber wie Politik hier gemacht wird, da bin ich ein bisschen skeptisch dem gegenüber. Weil viel Parteipolitik betrieben wird. Es geht nicht wirklich in erster Linie darum, die Probleme, die in der Gesellschaft vorhanden sind, sachlich zu lösen bzw. Lösungen zu finden, sondern eher versucht wird für die eigene Partei Werbung zu machen und zu sagen: ,Das, was von meiner Partei kommt, ist besser. ““ (I9, männlich, *1993, seit 2007 in Ö.) 
„,Wir Flüchtlinge werden in der Politik von manchen Politiker/inne/n wie der Innenminister, Außenminister und auch der FPÖ ausgenutzt. ... Das ist einfach eine Strategie von den Politikern, Politikerinnen, dass sie die Menschen mit Asylpolitik beschäftigen, dass die Leute da fokussiert werden und andere Dinge nicht sehen können, soziale Probleme, gesundheitliche Probleme - wie soll ich sagen? Bildungsprobleme, Studienprobleme, Kinderbeihilfe usw. “ (I17, männlich, *1997, seit 2013 in Ö.)

„, Aber im Asylbereich ist es schwieriger geworden, vor allem für Afghanen. Ich bin überhaupt nicht damit einverstanden, weil Afghanistan ist wirklich unsicher. Wenn Afghanistan sicher wäre, würde ich heute nicht hier leben, und ich wäre dort und diente meinen Landesleuten, aber Afghanistan ist leider kein Land zum Leben .... “ (I48, männlich, *1991, seit 2014 in Ö.)

\subsubsection{Trennung von Staat bzw. Politik und Religion}

Fast alle Respondent/inn/en sprachen sich explizit für eine Trennung von Staat und Religion aus. Ein Grund hierfür ist etwa das Negativbeispiel in Afghanistan oder anderen Ländern, wo durch die enge Verbindung von Politik und Religion Konflikte entstehen. Es wird außerdem befürchtet, dass andere Religionen benachteiligt würden. Stattdessen bevorzugen die Interviewten eine tolerantere Gesellschaft, in der Religionsfreiheit gewährleistet ist.

\section{Religion als moralische Institution}

Einige wenige meinen, dass die Religion eventuell einen positiven Einfluss auf die Politik ausüben könnte, als „moralische Instanz“ zum Beispiel, und die Politiker/ innen dabei unterstützen kann, moralisch einwandfreie Gesetze zu erlassen. Allerdings wird auch von dieser Gruppe von Respondent/inn/en bestätigt, dass die Umsetzung in der Praxis problematisch ist.

„,... wir haben also immer Religion als eine Art moralische Institution verstanden. Also natürlich diese Werte, welche Religion vermittelt, sind eigentlich sehr positiv, was Gerechtigkeit betrifft, was Frieden betrifft, was Zusammenarbeit betrifft, die Einhaltung bestimmter Regeln. Also die sind sehr, sehr positiv, dagegen ist niemand. Allerdings ist es so, man kann diese Werte als eine Art moralische Institution verstehen, die eigentlich politisches Leben gewissermaßen positiv beeinflussen kann. Dagegen ist keiner. Aber dass die Gesellschaft nach Normen und Pflichten der Religion handeln muss, das ist etwas Inakzeptables. " (I37, männlich, *1952, seit 1988 in Ö.)

In einem Interview wurde auch angedeutet, dass demokratische Strukturen für Österreich zwar passend sind, in anderen Ländern eine andere Regierungsform aber möglicherweise geeigneter wäre:

„In meinem Heimatland darf man manche Dinge nicht, weil die Regierung mit der Religion zusammen ist. Manche Dinge gehen nicht. Zum Beispiel können Männer 
keine Männer heiraten oder Frauen Frauen. Und hier geht das und ich glaube für die Leute in Österreich sind diese Sachen gut und für die Leute in meiner Heimat sind andere Sachen gut. " (I2, männlich, *1998, seit 2015 in Ö.)

Auf eine mögliche Dominanz der Religion gegenüber der Politik wurde ebenfalls hingewiesen:

„Es gibt auch einen Zwiespalt, welche Gesetze als wichtiger angesehen werden, also staatliche oder religiöse Gebote. Manche würden sich schon wünschen, dass die Religion eine stärkere Rolle spielt und es z.B. eine religiöse Partei gibt. Grundsätzlich Demokratie ja, aber die Religion spielt doch noch eine wichtige Rolle. “(E13)

\section{Klare Trennung von Politik und Religion}

Die überwiegende Mehrheit befürwortet eine strikte Trennung von Religion und Politik.

„Jede/r sollte das Recht haben, seine/ihre Meinung zu äußern, unabhängig davon, ob religiös oder nicht. " (I48, männlich, *1991, seit 2014 in Ö.)

„I: Ihrer Meinung nach, sollte ein religiöser Mensch in der Regierung und in der Politik was zum Sagen haben? B: Ja, das Recht sollte dieser Mensch haben, aber sieler darf die Politik mit der Religion nicht vermischen. “ (I51, männlich, *1993, seit 2010 in Ö.)

Ein Argument hierfür ist, dass eine Miteinbeziehung der Religion in die Politik zur Benachteiligung von anderen Religionen führen könnte. Das geht damit einher, dass Religion als Privatsache angesehen wird, während die Politik das Leben aller Menschen regelt, egal welcher Religion sie angehören, woran sie glauben und wie sie diesen Glauben praktizieren.

„Religion sollte sich nicht in Gesetze einmischen, weil es gibt immer die Leute, die Religion benützen. Eigentlich finde ich, Religion ist eine Privatsache. " (I17, männlich, *1997, seit 2013 in Ö.)

„, Staat und Religion sollten auf jeden Fall getrennt sein, denn nur so kann man Religionsfreiheit gewährleisten. " (I57, weiblich, *1966, seit 2015 in Ö.)

Die Trennung von Religion und Politik wird von den Respondent/inn/en unter anderem mit dem Hinweis darauf befürwortet, dass der Konflikt in Afghanistan u.a. durch die enge Beziehung zwischen Religion und Staat bedingt ist. Für sie passt religiös orientierte Politik nicht zur heutigen säkularen Weltordnung.

„... wenn man ein bisschen in der Politik gearbeitet hat, weiß man ganz genau, die heutige Weltordnung ist säkular. ... Eine Gesellschaft kann nur nach einer Ordnung existieren. Und hat man eigentlich versucht, immer einen sogenannten islamischen Staat zu gründen. ... In der Geschichte des Islams hat es niemals einen 
islamischen Staat gegeben. ... Und aus dem Grund vieles, was heute behauptet wird, kann mit dem modernen Staat eigentlich nicht vereinbart sein. Aus dem Grund ich bin 100\% für eine säkulare Ordnung, für einen demokratischen Staat und vor allem für Demokratie, für freie Entscheidung eines Bürgers. D.h., die Menschen sollten eigentlich Bürger eines Staates werden, nicht als Untertanen, nicht irgendwie als Schafe, die einen Hirten brauchen. " (I37, männlich, *1952, seit 1988 in Ö.)

„Es ist keine gute Idee, weil der Staat sollte nichts mit der Religion zu tun haben. Der Staat muss das Land weiterbringen und seine Bevölkerung schützen. “ (I59, weiblich, *1999, seit 2016 in Ö.)

„Das muss wirklich getrennt sein. Wir waren schon damals dafür und man sieht einfach in den islamischen Ländern, dass es einfach nicht funktioniert. Eben weil ... es viele verschiedene Auslegungsmöglichkeiten gibt, deshalb. " (I45, weiblich, *1987, seit 1992 in Ö.)

Während die meisten die Trennung von Religion und Politik augenscheinlich nur auf den Islam beziehen, wird diese Idee aber manchmal weitergedacht, so dass auch eine striktere Trennung des österreichischen Staats vom Christentum gefordert wird bzw. eine generelle Säkularisierung.

„Nein, ich finde Religion und Staat sollte richtig geteilt werden. Ich finde es auch zu viel, wenn in einem Klassenraum ein Kreuz steht, weil wenn da jetzt nicht ein jüdisches Zeichen steht, warum dann ein Kreuz? Ja, ich finde, das sollte total getrennt werden, aber wenn es so um so Sachen geht wie Kopftuch, sollte man den Leuten schon die Freiheit geben. " (I12, weiblich, *1999, seit 2006 in Ö.)

\section{Erwartungen an den österreichischen Staat und staatliche Einrichtungen}

Hinsichtlich der Leistungen durch den Staat Österreich sind die meisten Befragten zufrieden.

„Ich habe keine Erwartungen von der österreichischen Regierung. Ich bin zufrieden und habe wirklich alles, was ich brauche. Alles hängt jetzt von mir ab! “ (I52, männlich, *1993, seit 2009 in Ö.)

Viele Erwartungen der Afghan/inn/en an den österreichischen Staat hängen mit ihrer Integration zusammen. Es wird erwartet, dass der Staat Strukturen schafft, die es den Afghan/inn/en ermöglichen, sich zu integrieren, insbesondere auf dem Arbeitsmarkt. Im Zusammenhang damit wünschen sich auch viele, dass mehr Bildungs- und Ausbildungsangebote für Asylberechtigte geschaffen werden, damit sie rasch auf eigenen Beinen stehen können.

„Ich wünsche mir, dass die Regierung den Zugang zum Arbeitsmarkt auch für die Flüchtlinge aufmacht. Derzeit findet man sehr schwer einen Job. Wenn von allen Seiten gesagt wird, dass man arbeitet und wir uns für einen Job bewerben, ist es 
schwierig, weil sobald sie sehen, dass man aus Afghanistan kommt, sagen sie, es geht leider nicht. Ich wünsche mir von der Regierung, dass solche Diskriminierung aufgrund der Herkunft beseitigt wird. " (I56, männlich, *1999, seit 2015 in Ö.)

„Ich finde Österreich, ist ein starkes Land, mit vielen Möglichkeiten, wo auch die Migranten viele Freiheiten haben. Ich finde es sollte mehr Beschäftigung geben und auch vor allem für jene mit einer bereits abgeschlossenen Ausbildung. " (I57, weiblich, *1966, seit 2015 in Ö.)

\subsubsection{Politische Tätigkeiten und Engagement}

\section{Wichtigkeit der Mitgestaltung der österreichischen Gesellschaft}

In den Interviews wurde eine Vielzahl an Einstellungen bezüglich der Mitgestaltung der österreichischen Gesellschaft und der politischen Teilnahme verbalisiert. Es wurde auch betont, dass kein Interesse an Politik bestehe oder dass man Politik generell vermeiden wolle, da es dadurch nur zu Konflikten und zu keinen positiven Entwicklungen komme. Andererseits würde sich eine Gruppe von Respondent/inn/en gerne in der österreichischen Politik engagieren, in Parteien mitwirken oder an politischen Aktionen teilnehmen, wird aber aufgrund der fehlenden österreichischen Staatsbürgerschaft davon abgehalten. Eine andere Gruppe wiederum möchte weniger politisch aktiv sein, aber stattdessen die österreichische Gesellschaft durch wohltätige Aktivitäten mitgestalten.

„Ja, ich habe vor in Österreich zu bleiben. Und ich versuche, einige Sachen zu ändern. Hier zu leben und zu zeigen, dass ich hier zuhause bin und denen zu helfen, die es schwieriger haben. Damit sie sich auch hier zuhause fühlen. " (I10, weiblich, *1997, seit 2006 in Ö.)

Wahlrecht

Zwar dürfen meisten Respondent/inn/en noch nicht wählen, der Großteil aber wünscht sich, die Zukunft des Landes mitbestimmen zu können. Jene, die das Wahlrecht bereits erhalten haben, machen davon auch Gebrauch.

„Ich bin aktiv immer bei Wahlen. Wir gehen immer. “ (I23, weiblich, *1949, seit 1988 in Ö.)

„, Wir leben in diesem Land, wir haben eine Stimme, warum sollten wir stattdessen zuhause bleiben, weil es angenehmer ist?!" (I10, weiblich, *1997, seit 2006 in Ö.)

Ein Experte deutete außerdem auf den ausgeprägten Willen der Bevölkerung in Afghanistan hin, sich allen Gefahren zum Trotz für die Demokratie und ihr persönliches Wahlrecht einzusetzen. 
„Die Taliban haben zum Beispiel bedroht, wer zu Wahllokalen geht, wird seine Hand dann abgehackt. Oder sein Finger abgehackt. Und sie sind trotzdem gegangen und versucht eben ihre Stimme abzugeben. “ (E6)

Nur in Einzelfällen wird festgestellt, dass ein potentielles Wahlrecht nicht von Interesse ist und der/die Betreffende auch nicht davon Gebrauch machen würden.

„Ich wähle gar nicht. Aber ich darf nicht, zuerst. ... Ich gehe nicht. Bringt gar nichts. “(I11, männlich, *1988, seit 2011 in Ö.)

Es besteht aber auch ein ausgeprägtes Unwissen bezüglich der Politik in Österreich, was viele daran hindert, sich zu engagieren, obwohl eventuell ein Interesse daran bestünde.

„Ja, weil ich liebe schon folgen Politik. Obwohl ist es sehr schwer für mich, ich konnte nicht lernen von österreichischen Politiker gut kennen und wissen. Ja, das ist wegen Sprache, Lesen und Schreiben, das ist Problem, finde ich. " (I35, weiblich, *1974, seit 2004 in Ö.)

\section{Politisches Engagement}

In vielen Interviews ging es auch darum, sich abgesehen vom Gebrauch des Wahlrechts, politisch zu engagieren, zum Beispiel durch die Mitarbeit in einer Partei oder durch politisch neutrale Tätigkeiten, etwa als Wahlleiter/in. Es wird vor allem argumentiert, dass das politische Engagement der Integration förderlich ist und dass man dieses ausbauen oder fördern sollte.

„Für Integration dieser Menschen wäre auch sehr wichtig, auch sie zu motivieren in den Parteien Österreichs teilzuhaben. “ (I32, weiblich, *1975, seit 1995 in Ö.)

„,Deswegen versuche ich, dass ich schnell eine Staatsbürgerschaft bekomme, dann habe ich alle Möglichkeiten. Und später die Kinder sind erwachsen, vielleicht dann kann ich schon weitergehen. Ja, ein bisschen, in einer Partei vielleicht arbeiten. " (I35, weiblich, *1974, seit 2004 in Ö.)

\section{Teilnahme an Demonstrationen}

Die Teilnahme der Respondent/inn/en an Demonstrationen betrifft weitgehend Demonstrationen zu Themen wie Menschenrechte, insbesondere im Flüchtlingsbereich, die Situation in Afghanistan ebenso wie die internationale politische Lage. Es wurde aber betont, dass es den Respondent/inn/en wichtig ist, dass die Demonstrationen friedlich verlaufen.

„Ich bin immer dabei bei Demonstrationen! Letztens gab es ja wegen den Flüchtlingen eine Demonstration, da habe ich auch teilgenommen. Ich beteilige mich, wo ich mich beteiligen kann. " (I10, weiblich, *1997, seit 2006 in Ö.)

„Ich bin ein sozialer Mensch und mag es bei solchen Aktivitäten dabei zu sein und teilzunehmen. Wenn ich denke, dass diese Veranstaltungen berechtigt sind, 
dann ich werde ich auch versuchen, diese Menschen zu unterstützen. Aber wenn ich der Meinung bin, dass solche Veranstaltungen nur zum Chaos führen und das friedliche Zusammenleben stören, dann werde ich versuchen mich daran nicht zu beteiligen. " (I48, männlich, *1991, seit 2014 in Ö.)

Der Großteil der Respondent/inn/en nimmt allerdings nicht an Demonstrationen oder ähnlichen Aktivitäten teil, was die Expert/inn/en bestätigen.

„Sie sind schon zum Teil recht zurückhaltend, gerade mit dem Demonstrieren gehen. Das machen manche, aber sehr viele auch nicht. Da sind dann bei Hochzeiten und so was mehr Leute als bei politischen Kundgebungen. " (E2)

\section{Soziales Engagement}

Einige engagieren sich nicht direkt politisch, sondern möchten durch Mithilfe in Vereinen oder sozialen Organisationen zu einer Verbesserung der Situation beitragen, den österreichischen Staat unterstützen und anderen Flüchtlingen helfen.

„Ich engagiere mich nicht politisch und ich möchte mich eigentlich eher von Politik fernhalten. ... ehrenamtlich was machen, indem man Leuten hilft oder so, das mach ich wirklich gerne. ... Im sozialen Bereich ja, aber nicht in der Politik. ... sobald ich mit jemandem drüber diskutiere, dann führt das immer zu Streit. “ (I45, weiblich, *1987, seit 1992 in Ö.)

„Und auch was die sozialen Aktivitäten angeht, ich bin auch da aktiv. Ich gehe zwischendurch zur einen Organisation und verteile Kleidung. " (I54, männlich, *1996, seit 2015 in Ö.)

\section{Engagement über Musik und Literatur}

In einigen Fällen wird auch Kunst in Form von Musik oder dem Schreiben von Büchern als Weg gesehen, um die Gesellschaft mitzugestalten.

„Ich rappe auch gegen Politik .... Also für die Freiheit. “ (I16, männlich, *1997, seit 2012 in Ö.)

„Ich schreibe lieber, ist das so, dass ich würde es ... Man erreicht mehr Leute, wenn man eben ... die politischen Debatten sind gut, aber mit einem Buch erreicht man mehr, finde ich. “ (I30, männlich, *1953, seit 1984 in Ö.)

\section{Abwendung von der Politik}

Manche wenden sich allerdings ganz von der Politik ab, nachdem sie im Heimatland oder Transitländern schlechte Erfahrungen gemacht haben und die Politik für die Situation in Afghanistan verantwortlich machen.

„Nein, ich persönlich mag die Politik nicht, weil ich eine schlechte Erfahrung mit ihr habe. Politik ist nur mit schlechten Sachen behaftet und ich mag es gar nicht, dass sich jemand in meine Angelegenheit einmischt, und ich selbst mische mich nirgendwo ein. “ (I59, weiblich, *1999, seit 2016 in Ö.) 
„Nein, ich mag Politik nicht! ... Mir wurde immer gesagt, dass die Politik reine Drecksarbeit ist und dass alles Schlimme von der Politik und den Politikern kommen würde. Die Politik hat nur für diejenigen etwas zu bieten, die ganz oben sind. Unsere Meinung ist nicht wichtig, weil wir eine niedrige Position haben. “ (I55, männlich, *1998, seit 2014 in Ö.)

Politische Tätigkeiten in der Familie oder im Freundeskreis

In den Interviews wird betont, dass die friedliche Diskussion im Vordergrund steht sowie dass man Streit verhindern will. Aus diesem Grund vermeiden manche Befragte politische Themen zur Gänze oder sehen Diskussionen sehr kritisch und mit gehöriger Vorsicht. Manche betrachten solche Diskussionen gar als Einmischung in ihr Leben oder in das Leben anderer. Andere wiederum meinen, sie hätten niemanden, mit dem sie darüber reden könnten. Bei politischen Diskussionen mit Familie und Freunden wird häufig über den Sicherheitsaspekt im Heimatland gesprochen oder über die Situation für Afghan/inn/en und generell für Flüchtlinge in Österreich.

„, Also mit der Familie gab es schon einige Diskussionen, denn jeder steht irgendwie zu seiner Meinung. Auch im Kurs wird auch das eine oder andere Mal darüber diskutiert, jedoch schaffen wir es auch immer wieder, es auf friedliche Art und Weise zu lösen. Ich glaube, über dieses Thema wird es immer Auseinandersetzungen geben. " (I57, weiblich, *1966, seit 2015 in Ö.)

„Ich diskutiere mit niemandem über Politik. Das ist sinnlos, das ist das peinlichste Zeitverschwendung im Leben. Weil jeder hat seine eigene Meinung. Warum soll ich meine Zeit verschwenden? Ich respektiere einfach, was er denkt, was sie denkt. " (I3, männlich, *1992, seit 2014 in Ö.) 